\title{
Stronger Bounds for Weak Epsilon-Nets in Higher Dimensions*
}

\author{
Natan Rubin ${ }^{\dagger}$
}

April 27, 2021

\begin{abstract}
Given a finite point set $P$ in $\mathbb{R}^{d}$, and $\epsilon>0$ we say that $N \subseteq \mathbb{R}^{d}$ is a weak $\epsilon$-net if it pierces every convex set $K$ with $|K \cap P| \geq \epsilon|P|$.

Let $d \geq 3$. We show that for any finite point set in $\mathbb{R}^{d}$, and any $\epsilon>0$, there exist a weak $\epsilon$-net of cardinality $O\left(\frac{1}{\epsilon^{d-1 / 2+\gamma}}\right)$, where $\gamma>0$ is an arbitrary small constant.

This is the first improvement of the bound of $O^{*}\left(\frac{1}{\epsilon^{d}}\right)$ that was obtained in 1994 by Chazelle, Edelsbrunner, Grigni, Guibas, Sharir, and Welzl for general point sets in dimension $d \geq 31$
\end{abstract}

\footnotetext{
${ }^{*}$ The project leading to this application has received funding from European Research Council (ERC) under the European Unions Horizon 2020 research and innovation programme under grant agreement No. 678765.

${ }^{\dagger}$ Email: rubinnat.ac@gmail.com. Ben Gurion University of the Negev, Beer-Sheba, Israel. Ralph Selig Career Development Chair in Information Theory.

${ }^{1} O^{*}(\cdot)$-notation hides multiplicative factors that are polylogarithmic in $\log 1 / \epsilon$.
} 


\section{Introduction}

Transversals and $\epsilon$-nets. Given a family $\mathcal{K}$ of geometric ranges in $\mathbb{R}^{d}$ (e.g., lines, triangles, or convex sets), we say that $N \subset \mathbb{R}^{d}$ is a transversal to $\mathcal{K}$ (or $N$ pierces $\mathcal{K}$ ) if each range $K \in \mathcal{K}$ is pierced by at least one point of $N$. Given an underlying set $P$ of $n$ points, we say that a range $K \in \mathcal{K}$ is $\epsilon$-heavy if $|P \cap K| \geq \epsilon n$. We say that $N$ is an $\epsilon$-net for $\mathcal{K}$ if it pierces every $\epsilon$-heavy range in $\mathcal{K}$. We say that such a set $N$ is a strong $\epsilon$-net for $\mathcal{K}$ if $N \subset P$, that is, the points of the net are drawn from the underlying point set $P$. Otherwise (i.e., if $N$ includes additional points outside $P$ ), we say that $N$ is a weak $\epsilon$-net.

The study of $\epsilon$-nets was initiated by Vapnik and Chervonenkis [57], in the context of Statistical Learning Theory. Following a seminal paper of Haussler and Welzl [35], $\epsilon$-nets play a central role in Discrete and Computational Geometry [41]. For example, bounds on $\epsilon$-nets determine the performance of the best-known algorithms for Minimum Hitting Set/Set Cover Problem in geometric hypergraphs [7, 15, 25, 29], and the transversal numbers of families of convex sets [3, 4, 6, 39].

Informally, the cardinality of the smallest possible $\epsilon$-net for the range set $\mathcal{K}$ determines the integrality gap of the corresponding transversal problem - the ratio between (1) the size of the smallest possible transversal $N$ to $\mathcal{K}$ and (2) the weight of the "lightest" possible fractional transversal to $\mathcal{K}[6,3,29]$.

Haussler and Welzl [35] proved in 1986 the existence of strong $\epsilon$-nets of cardinality $O\left(\frac{1}{\epsilon} \log \frac{1}{\epsilon}\right)$ for families of simply-shaped, or semi-algebraic geometric ranges in $d$-space, for a fixed $d>0$ (e.g., lines, boxes, spheres, halfspaces, or simplices), by observing that their induced hypergraphs have a bounded Vapnik-Chervonenkis dimension (so called VC-dimension).$^{2}$ While the bound is generally tight for set systems with a bounded VC-dimension [40], tight $o\left(\frac{1}{\epsilon} \log \frac{1}{\epsilon}\right)$-size constructions were discovered for discs in $\mathbb{R}^{2}$, halfplanes in $\mathbb{R}^{2}$ and halfspaces in $\mathbb{R}^{3}\left[25\right.$, 40, 45], and rectangles in $\mathbb{R}^{2}$ and boxes in $\mathbb{R}^{3}[7,50$. We refer the reader to a recent state-of-the-art survey [48].

It had long been conjectured that all the "natural" geometric instances, that involve simplyshaped geometric ranges in a fixed-dimensional Euclidean space $\mathbb{R}^{d}$, admit a strong $\epsilon$-net of cardinality $O(1 / \epsilon)$. The conjecture was refuted, for the particular case of line ranges, in 2010 by Alon [2]. Pach and Tardos [50] subsequently demonstrated that the multiplicative term $\Theta(\log 1 / \epsilon)$ is necessary for halfspaces in dimension higher than 3 .

Weak $\epsilon$-nets for convex sets. In sharp contrast to the case of simply-shaped ranges, no constructions of small-size strong $\epsilon$-nets exist for general families of convex sets in $\mathbb{R}^{d}$, for $d \geq 2$. For example, given an underlying set of $n$ points in convex position in $\mathbb{R}^{2}$, any strong $\epsilon$-net with respect to convex ranges must include at least $n-\epsilon n$ of the points. Informally, this phenomenon can be attributed to the fact that the VC-dimension of a geometric set system is closely related to the description complexity of the underlying ranges, and it is unbounded for general convex sets. Nevertheless, Bárány, Füredi and Lovász [13. observed in 1990 that families of convex sets in $\mathbb{R}^{2}$ still admit weak $\epsilon$-nets of cardinality $O\left(\epsilon^{-1026}\right)$. Alon, Bárány, Füredi, and Kleitman [1] were the first to show in 1992 that families of convex sets in any dimension $d \geq 1$ admit weak $\epsilon$-nets whose cardinality is bounded in terms of $1 / \epsilon$ and $d$. The subsequent study and application of weak $\epsilon$-nets bear strong relations to convex geometry, including Helly-type, Tverberg-type, and Selection Theorems; see [42, Sections 8 - 10] for a comprehensive introduction.

Weak $\epsilon$-nets and the Hadwiger-Debrunner Problem. Alon and Kleitman [6] used the boundedness

\footnotetext{
${ }^{2}$ The constant hidden within the $O(\cdot)$-notation is specific to the family of geometric ranges under consideration, and is proportional to the VC-dimension of the induced hypergraph.
} 
of weak $\epsilon$-nets to confirm a long-standing $(p, q)$-conjecture by Hadwiger and Debrunner [33]. To this end, we say that a family $\mathcal{K}$ of convex sets satisfies the $(p, q)$-property if any its $p$-size subfamily $\mathcal{K}^{\prime} \subset \mathcal{K}$ contains a $q$-size subset $\mathcal{K}^{\prime \prime} \subset \mathcal{K}$ with a non-empty common intersection $\bigcap \mathcal{K}^{\prime \prime} \neq \emptyset$. Hadwiger and Debrunner conjectured that for every positive integers $p, q$ and $d$ that satisfy $p \geq q \geq d+1$, there exists an integer $C_{d}(p, q)<\infty$ such that the following statement holds: Any family $\mathcal{K}$ of convex sets in $\mathbb{R}^{d}$ with the $(p, q)$-property admits a transversal by at most $C_{d}(p, q)$ points. Note that the celebrated Helly Theorem yields a transversal by a single point in the case $p=q=1$ whenever $|\mathcal{K}| \geq d+1$. Showing good quantitative estimates for the Hadwiger-Debrunner numbers $C_{d}(p, q)$ is a formidable open problem which requires tight asymptotic bounds for weak $\epsilon$-nets; see the latest study by Keller, Smorodinsky and Tardos [39], and the concluding discussion in Section 6 .

Very recently, lower bounds for several of the above questions - including strong and weak $\epsilon$-nets with respect to line ranges [12, and the 2-dimensional Hadwiger-Debrunner numbers $C_{2}(p, q)[38$ ] - were improved using the novel combinatorial machinery of hypergraph containers [11, 53].

Weak $\epsilon$-nets, Radon numbers, and the fractional Helly theorem. Alon, Kalai, Matoušek, and Meshulam [4] studied the above problems in a more general setting of abstract hypergraphs that are closed under intersections. They showed that the existence of weak $\epsilon$-net (whose size is bounded in $1 / \epsilon$ ) can be combinatorially deduced from the so called fractional Helly property. More recently, Holmsen and Lee [36, 37] showed that the latter property is a purely combinatorial consequence of the bounded Radon number, which is equal to $d+2$ for convex sets in $\mathbb{R}^{d}$; also see a related study [46].

Bounds on weak $\epsilon$-nets. For any $\epsilon>0$ and $d \geq 0$, let $f_{d}(\epsilon)$ be the smallest number $f>0$ so that, for any underlying finite point set $P$, one can pierce all the $\epsilon$-heavy convex sets using only $f$ points in $\mathbb{R}^{d}$. It is an outstanding open problem in Discrete and Computational geometry to determine the true asymptotic behaviour of $f_{d}(\epsilon)$ in dimensions $d \geq 2$. As Alon, Kalai, Matoušek, and Meshulam noted in 2001: "Finding the correct estimates for weak $\epsilon$-nets is, in our opinion, one of the truly important open problems in combinatorial geometry" [4].

Alon, Bárány, Füredi, and Kleitman [1] (see also [6]) used Tverberg-type results to show that $f_{d}(\epsilon)=O\left(1 / \epsilon^{d+1-1 / \alpha_{d}}\right.$ ) (where $0<\alpha_{d}<1$ is a selection ratio which is fixed for every $d$ ), and $f_{2}(\epsilon)=O\left(1 / \epsilon^{2}\right){ }^{3}$ The bound in higher dimensions $d \geq 3$ has been subsequently improved in 1993 by Chazelle et al. [22] to roughly $O^{*}\left(\frac{1}{\epsilon^{d}}\right)$ (where $O^{*}(\cdot)$-notation hides multiplicative factors that are polylogarithmic in $\log 1 / \epsilon$ ). Though the latter construction was somewhat simplified in 2004 by Matoušek and Wagner [44] using simplicial partitions with low hyperplane-crossing number [43], no study in the subsequent 25 years came close to tackling the notorious " $\frac{1}{\epsilon^{d}}$-barrier" for general families of convex sets and arbitrary finite point sets, in any dimension $d \geq 2$.

In view of the best known lower bound of $\Omega\left(\frac{1}{\epsilon} \log ^{d-1}\left(\frac{1}{\epsilon}\right)\right)$ for $f_{d}(\epsilon)$ due to Bukh, Matoušek and Nivasch [16], it still remains to settle whether the asymptotic behaviour of this quantity substantially deviates from the long-known "almost- $(1 / \epsilon)$ " bounds on strong $\epsilon$-nets (e.g., for lines and triangles in $\mathbb{R}^{2}$ or simplices in $\left.\mathbb{R}^{d}\right)$ ? The only interesting instances in which the gap has been nearly closed, involve essentially lower-dimensional distributions of points [22, 17, 5]. For example, Alon, Kaplan, Nivasch, Sharir, and Smorodinsky [5] showed in 2008 that any finite point set in a convex position in $\mathbb{R}^{2}$ allows for a weak $\epsilon$-net of cardinality $O(\alpha(\epsilon) / \epsilon)$ with respect to convex sets, where $\alpha(\cdot)$ denotes the inverse Ackerman function.

\footnotetext{
${ }^{3}$ An outline of the planar $f_{2}(\epsilon)=O\left(1 / \epsilon^{2}\right)$ bound can be found in a popular textbook by Chazelle [21].
} 
A FOCS 2018 paper by the author [51] made the first step to breaching the infamous $\frac{1}{\epsilon^{d}}$-barrier by showing that $f_{2}(\epsilon)=O\left(\frac{1}{\epsilon^{3 / 2+\gamma}}\right)$, for any constant $\gamma>0$. Unfortunately, its (partly) ad-hoc machinery did not directly apply to dimensions $d \geq 3$.

Our result. The present study offers the first $o\left(1 / \epsilon^{d}\right)$-size construction in any dimension $d \geq 3$.

Theorem 1.1. Let $d \geq 3$. Then we have that

$$
f_{d}(\epsilon)=O\left(\frac{1}{\epsilon^{d-1 / 2+\gamma}}\right)
$$

where $\gamma>0$ is an arbitrary small constant.

That is, for any underlying set $P$ of $n$ points in $\mathbb{R}^{d}$, and any $\epsilon>0$, one can construct a weak $\epsilon$-net with respect to convex sets whose cardinality is $O\left(\frac{1}{\epsilon^{d-1 / 2+\gamma}}\right)$. Here $\gamma>0$ is am arbitrary small constant, and the $O(\cdot)$-notation may hide constant factors that depend on $\gamma$.

Our proof of Theorem 1.1 is fully constructive. It combines an array of powerful tools such as the so called Second Selection Theorem [1] for dense geometric hypergraphs, Matoušek's Simplicial Partition Theorem [43], strong $\epsilon$-nets [35, cuttings in hyperplane arrangements [23], and upper bounds on the complexity of many cells in hyperplane arrangements [ 8 , 9].

Notably, we establish a variant of the Second Selection Theorem for simplices that are induced by the points of an unknown convex set, and then use it in $\mathbb{R}^{d-1}$ in order to derive a basic reduction of the weak $\epsilon$-net problem to a sequence of 1-dimensional strong $\lambda$-nets, for $\lambda=\Omega\left(\epsilon^{d}\right)$.

However, the main underlying idea of our proof is fairly simple: we gradually construct a small auxiliary net $X \subset \mathbb{R}^{d}$, of $o\left(1 / \epsilon^{d}\right)$ points, with the property that every $\epsilon$-heavy convex set $K$ that is missed by $X$, is "close enough" (in a certain combinatorial sense) to a hyperplane: The set $P_{K}:=P \cap K$ is largely determined by "almost any" $d$-tuple of its points in $\left(\begin{array}{c}P_{K} \\ d\end{array}\right)$. This property is better quantified using such spatial decomposition tools as simplicial partitions, and cuttings in hyperplane arrangements, which were previously used in the study of point-hyperplane incidences; see, e.g., [24] and [42, Section 4].

The eventual net combines the following elementary ingredients: (1) 1-dimensional $\lambda$-nets, for $\lambda \gg \epsilon^{d}$, which are constructed within few vertical lines and with respect to carefully chosen point sets, and (2) strong $\hat{\epsilon}$-nets with respect to convex $(2 d)$-hedra in $\mathbb{R}^{2}$, for $\epsilon^{d} \ll \hat{\epsilon} \ll \epsilon$.

Organization. The rest of the paper is organized as follows:

In Section 2 we provide a comprehensive overview of our approach and survey the key geometric machinery (namely, random sampling and strong $\epsilon$-nets, the Second Selection Theorem, simplicial partitions, and arrangements of hyperplanes), and lay down the recursive framework that underlies our proof of Theorem 1.1 .

In Section 3 we obtain the basic reduction of the weak $\epsilon$-net problem to a sequence of 1dimensional $\lambda$-nets, for $\lambda=\Omega\left(\epsilon^{d}\right)$. To that end, we introduce a novel Multiple Selection Theorem 3.3 whose proof uses a non-trivial combination of Matoušek's Simplicial Partition Theorem and the Second Selection Theorem of Alon, Bárány, Füredi, and Kleitman [1].

In Section 4 we provide a net for so called vertically convex sets $K$ which cut out subsets $P_{K}=P \cap K$ with the following property: For the vast majority of the $(d+1)$-tuples in $\left(\begin{array}{c}P_{K} \\ d+1\end{array}\right)$, their projections onto $\mathbb{R}^{d-1}$ are in a convex position.

\footnotetext{
${ }^{4}$ As a rule, the constants proportionality in our $O(\cdot)$ and $\Omega(\cdot)$ notation heavily depend on the dimension $d$.
} 
In Section 5 we describe the net for the main scenario, which involves the $\epsilon$-heavy convex sets that are not vertically convex.

In Section 6 we wrap up the proof of Theorem 1.1 by deriving, and solving, a complete recurrence for the quantity $f_{d}(\epsilon)$. We then summarize the properties of our construction.

\section{Preliminaries}

The section is organized as follows. In Section 2.1 we lay put the basic notation in connection with convexity and simplicial hypergraphs, and introduce such fundamental results as the Second Selection Theorem [1] and Matoušek's Simplicial Partition Theorem [43. In Section 2.2 we summarize the main ideas behind our proof of Theorem 1.1. In Section 2.3 we review the essential machinery of random sampling, strong $\epsilon$-nets, arrangements of hyperplanes, and the complexity of various structures in arrangements. In Section 2.4 we lay out the recursive framework for our proof of Theorem 1.1.

\subsection{Geometric essentials}

Notation. In the sequel, we use $\operatorname{conv}(S)$ to denote the convex hull of any point set $S \subseteq \mathbb{R}^{d}$. For each point $p \in \mathbb{R}^{d}$ we use $p^{\perp}$ to denote its vertical projection to the copy of $\mathbb{R}^{d-1}$ that is spanned by the first $d-1$ coordinate axes. Accordingly, for a set $S \subset \mathbb{R}^{d}$ we denote $S^{\perp}:=\left\{p^{\perp} \mid p \in S\right\}$.

Let $\gamma_{0}>0$ be an arbitrary small constant to be determined in the sequel. (The choice of $\gamma_{0}$ may depend on the constant $\gamma>0$ in Theorem 1.1, and it is generally assumed to be much smaller than $d$, or any other positive constant to be found in this paper.) For any $x, y \in \mathbb{R}$ we denote $x \ll y$ whenever $x=O(y)$. Given that $y>1$, we denote $x \lll y$ whenever $x=O\left(y^{\gamma_{0}}\right)$. For $0<y<1$, we accordingly denote $x \lll y$ whenever $1 / y \lll 1 / x$.

General position. To simplify the exposition, we assume in what follows that the underlying point set $P$ in Theorem 1.1 is in a general position in $\mathbb{R}^{d}$ (as described, e.g., [42, Section 5] and [26]); in particular, no $d+1$ of them lie on the same hyperplane, and no $d$ of them determine a hyperplane that is parallel to any of the axes $x_{1}, \ldots, x_{d}$. A standard perturbation argument shows that such an assumption incurs no loss of generality $5^{5}$

Simplices. For any finite set $A$ and $k \geq 0$ let $\left(\begin{array}{l}A \\ k\end{array}\right)$ denote the collection of all the $k$-size subsets of $A$. If $A$ is a finite point set in general position in $\mathbb{R}^{d}$ and $0 \leq k \leq d$, then every $(k+1)$-set $S \in\left(\begin{array}{c}A \\ k+1\end{array}\right)$ determines a $k$-dimensional simplex $\operatorname{conv}(S)$ (or, shortly, a $k$-simplex) which supports a $k$-dimensional affine space $\operatorname{aff}(S)$ [42, Section 5]. For the sake of brevity, we identify the induced set of $\left(\begin{array}{c}|A| \\ k+1\end{array}\right)$ of $k$-dimensional simplices with $\left(\begin{array}{c}A \\ k+1\end{array}\right)$.

In what follows, we refer to any $k$-dimensional affine space as a $k$-dimensional plane, or shortly a $k$-plane; the $(d-1)$-dimensional planes in $\mathbb{R}^{d}$ are called hyperplanes.

Simplicial hypergraphs. Let $1 \leq k \leq d$. A $k$-uniform hypergraph in $\mathbb{R}^{d}$ is a pair $(V, E)$, where $V$ is a finite point set in a general position in $\mathbb{R}^{d}$, and $E \subseteq\left(\begin{array}{c}E \\ k\end{array}\right)$ is a family of $k$-subsets which can be identified with $(k-1)$-dimensional simplices whenever $1 \leq k \leq d+1$. In the vast majority of the cases, we have $k=d$, so $E$ consists of $(d-1)$-dimensional simplices.

For the sake of brevity, we identify our geometric hypergraph with its hyperedge set $E \subseteq\left(\begin{array}{l}V \\ k\end{array}\right)$ whenever the underlying vertex set $V$ is clear from the context (e.g., if $V$ is the underlying point set $P$, and $E=\left(\begin{array}{l}P \\ k\end{array}\right)$ is the complete $k$-uniform hypergraph).

\footnotetext{
${ }^{5} \mathrm{~A}$ weak $\epsilon$-net with respect to the perturbed point set, in a general position, would immediately yield such a net with respect to the original set.
} 

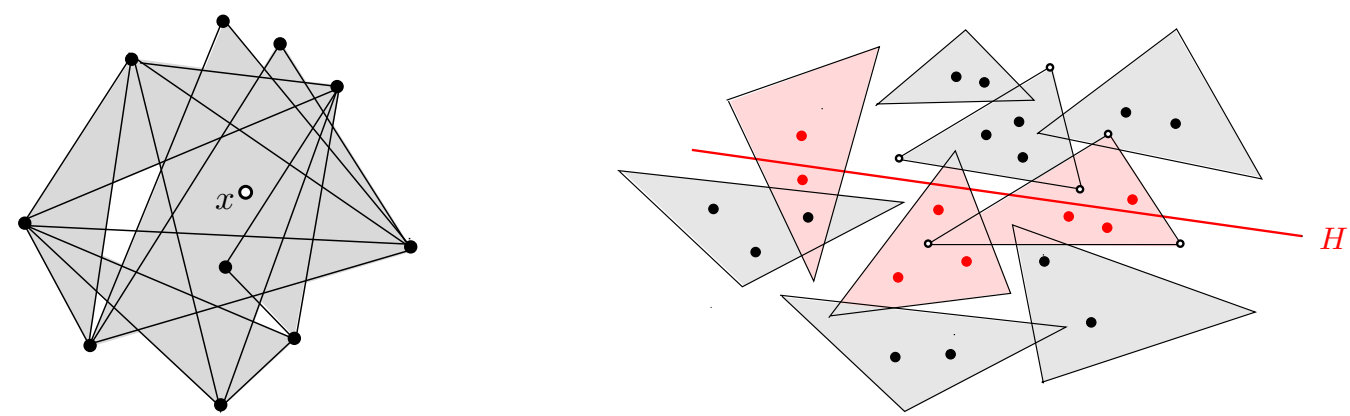

Figure 1: Left: The Second Selection Theorem. Depicted is a 3-uniform simplicial hypergraph $(V, E)$, in dimension $d=2$; the edge set $E$ consists of at least $h\left(\begin{array}{l}n \\ 3\end{array}\right)$ triangles, and the theorem yields a point $x \in \mathbb{R}^{2}$ that pierces $\Omega\left(h^{\beta_{2}}\left(\begin{array}{l}n \\ 3\end{array}\right)\right)$ of these triangles. Right: Matoušek's Simplicial Partition in dimension $d=2$ (the zone of the hyperplane $H$ is colored red).

Alon, Bárány, Füredi, and Kleitman [1] established the following crucial property of simplicial hypergraphs; see [42, Section 9] for a comprehensive exposition, and Figure 1)(left) for an illustration (in dimension 2).

Theorem 2.1 (The Second Selection Theorem). For any dimension $d \geq 1$ there exists a constant $\beta_{d}>0$ with the following property:

Let $(V, E)$ be a $(d+1)$-uniform simplicial hypergraph in $\mathbb{R}^{d}$ with $|E|=h\left(\begin{array}{l}n \\ d\end{array}\right)$ edges, then there is a point $x \in \mathbb{R}^{d}$ that pierces $\Omega\left(h^{\beta_{d}}\left(\begin{array}{c}n \\ d+1\end{array}\right)\right)$ of the $d$-simplices of $E[6$

Remark. The proof of our Multiple Selection Theorem 3.3 in Section 3.4 will require a slightly more general version of Theorem 2.1, in the form of Theorem 3.2 ,

Joins. Given a collection $\Pi \subseteq\left(\begin{array}{c}P \\ d-1\end{array}\right)$ of $(d-2)$-dimensional simplices, and a finite point set $X \subseteq \mathbb{R}^{d} \backslash P$ (in general position with respect to the underlying set $P$ ), we define their join as $\Pi * X:=\{\operatorname{conv}(\tau \cup\{p\}) \mid \tau \in \Pi, p \in X\}$. That is, $\Pi * X$ consists of all the $(d-1)$-dimensional simplices so that $d-1$ of their vertices form a hyperedge of $\Pi$, and the remaining vertex belongs to $X$.

Matoušek's Simplicial Partition Theorem. Let $P$ be a set of $n$ points in general position in $\mathbb{R}^{d}$, and $r>0$ be an integer. A simplicial $r$-partition $\mathcal{P}$ of $P$ is a collection $\left\{\left(P_{i}, \Delta_{i}\right) \mid 1 \leq i \leq r\right\}$ of $r$ pairs, where for each $1 \leq i \leq r$ we have that $P_{i} \subset P$ and $\Delta_{i}$ is a simplex of dimension at most $d$ in $\mathbb{R}^{d}$, so that the following properties are satisfied 7

1. $P=\biguplus_{i=1}^{r} P_{i}$.

2. For each $1 \leq i \leq r$ so that $P_{i} \neq \emptyset$, the cardinality $n_{i}:=\left|P_{i}\right|$ of $P_{i}$ satisfies $\lceil n / r\rceil \leq n_{i}<2\lceil n / r\rceil$.

3. For each $1 \leq i \leq r$, the set $P_{i}$ is contained in the relative interior of $\Delta_{i}$.

\footnotetext{
${ }^{6}$ The best known proof of the Second Selection Theorem yields the exponent $\beta_{d} \leq(2 d+2)^{(d+1)}$, and it is $\beta_{d} \leq(d+1)^{d+1}$ for $d+2>4$ is a prime; see [1, 14] and [42, Section 9]. Improved bounds for $\beta_{d}$ are known in dimension 2 (see, e.g., [28, 49]). Note that the constant of proportionality in this lower bound cannot be improved beyond $(d+1)^{-(d+1)}$ even for $h=1$ (the so called First Selection Lemma).

${ }^{7}$ For the sake of brevity, we require that each partition encompasses exactly $r$ sets $P_{i}$, for $1 \leq i \leq r$, some of which can be empty. Though a point of $P$ may belong to several simplices $\Delta_{i}$, for $1 \leq i \leq r$, it is assigned to exactly one of their respective sets $P_{i}$.
} 


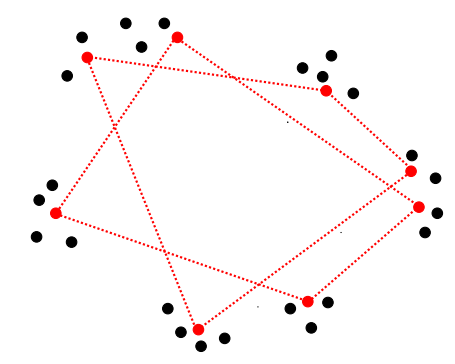

Figure 2: A vertically $\delta$-convex set $A$ in $\mathbb{R}^{3}$ - view in the 2-dimensional projection. At least $(1-\delta)\left(\begin{array}{c}|A| \\ 4\end{array}\right)$ of the 4 -subsets $B \in\left(\begin{array}{l}A \\ 4\end{array}\right)$ are vertically convex.

Definition. For each set $P_{i}$ in an $r$-partition $\mathcal{P}=\left\{\left(P_{i}, \Delta_{i}\right) \mid 1 \leq i \leq r\right\}$ of $P$, and each point $p \in P_{i}$, we refer to $\Delta_{i}$ as the ambient simplex of $p$.

For any hyperplane in $\mathbb{R}^{d}$, we say that a point $p \in P$ lies in the zone of $H$ within $\mathcal{P}$ if its ambient simplex is crossed by $H$.

Theorem 2.2 (The Simplicial Partition Theorem). For any $d \geq 2$ there is a constant $c_{d}$ with the following property. For any n-point set, and any $1 \leq r \leq n$, there is a simplicial $r$-partition $\left\{\left(P_{i}, \Delta_{i}\right) \mid 1 \leq i \leq r\right\}$ so that any hyperplane crosses at most $c_{d} r^{1-1 / d}$ of the simplices $\Delta_{i}$, for $1 \leq i \leq r$. See Figure 1 (right). Furthermore, such a partition $\mathcal{P}$ can be computed in time $\tilde{O}(n)]^{8}$

If the points of the underlying set $P$ are in a general position, then we can assume that all the simplices $\Delta_{i}$ in Theorem 2.2, are $d$-dimensional; furthermore, their vertices can be perturbed in a general position with respect to one another, and with respect to the point set $P$.

Vertical convexity. We say that an finite point set $A \subset \mathbb{R}^{d}$ is vertically convex if every point of $A^{\perp}$ lies on the boundary of conv $\left(A^{\perp}\right)$.

We say that a finite set $A \subset \mathbb{R}^{d}$, or cardinality $m$, is vertically $\delta$-convex if at least $(1-\delta)\left(\begin{array}{c}m \\ d+1\end{array}\right)$ of the $(d+1)$-subsets $B \in\left(\begin{array}{c}A \\ d+1\end{array}\right)$ are vertically convex; see Figure 2 .

\subsection{Overview}

Here is a hight level summary of our proof of Theorem 1.1 .

A reduction to 1-dimensional nets. We first describe a generic reduction of the weak $\epsilon$-net problem in $\mathbb{R}^{d}$, for $d \geq 2$, to a sequence of 1-dimensional $\Theta\left(\epsilon^{d}\right)$-nets with respect intervals of $\mathbb{R}^{1}$. Note the subset $P_{K}=P \cap K$ that is cut out by any $\epsilon$-heavy convex set $K$, determines a collection of $\left(\begin{array}{c}\left|P_{K}\right| \\ d\end{array}\right)=\Theta\left(\epsilon^{d} n^{d}\right)$ simplices.

Let $\ell$ be any vertical line that pierces $\alpha\left(\begin{array}{c}\left|P_{K}\right| \\ d\end{array}\right)$ of the simplices of $\left(\begin{array}{c}P_{K} \\ d\end{array}\right)$, for some constant $\alpha$ that depends only on the dimension $d$. Then, by the convexity of $K$, the interval $K \cap \ell$ must contain $\alpha\left(\begin{array}{c}\left|P_{K}\right| \\ d\end{array}\right)$ of the $\ell$-intercepts $\operatorname{conv}(\tau) \cap \ell$ of the simplices $\tau \in\left(\begin{array}{c}P_{K} \\ d\end{array}\right)$. See Figure 3 (left). Hence, we can pierce every such set $K$ by including in our net $N$ every $\left\lceil C \epsilon^{d} n^{d}\right\rceil$-th point of the

\footnotetext{
${ }^{8}$ The $\tilde{O}(x)$ notation hides asymptotic factors that are bounded by $x^{\gamma}$, for arbitrary small $\gamma>0$. The running time was improved by Chan 18 to $O(n \log n)$ (with high probability).
} 

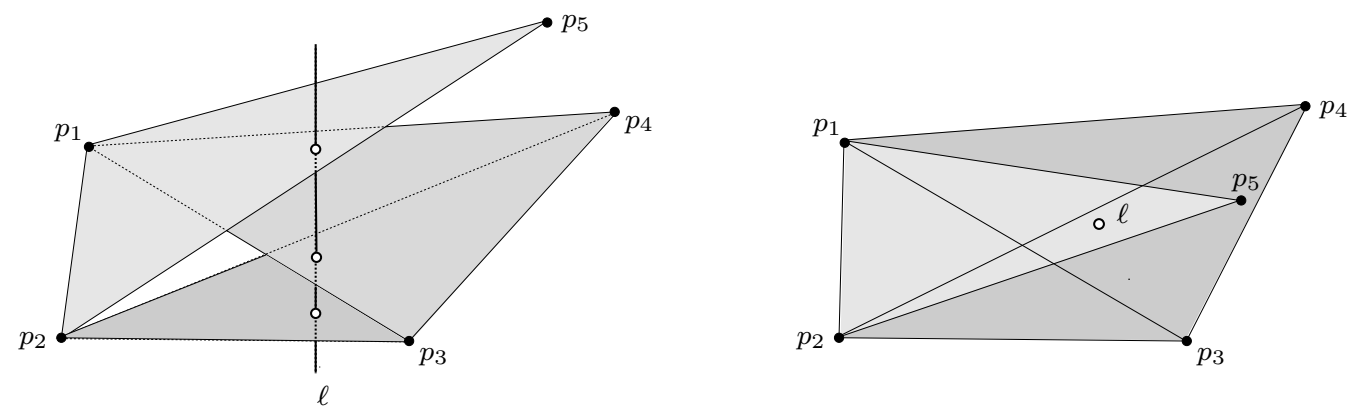

Figure 3: The reduction to 1-dimensional nets. The vertical line $\ell$ pierces the triangles $\triangle p_{1} p_{2} p_{5}, \triangle p_{3} p_{4} p_{1}, \triangle p_{3} p_{4} p_{2}$ that are determined by $p_{1}, \ldots, p_{5} \subset P_{K}$ (left) if and only if the projection of $\ell$ pierces the projections of the 3 triangles (right). Note that the $\ell$-intercepts of the triangles lie within $\operatorname{conv}\left(p_{1}, \ldots, p_{5}\right) \subset K \cap L$.

set $X_{\ell}=\left\{\operatorname{conv}(\tau) \cap \ell \mid \tau \in\left(\begin{array}{l}P \\ d\end{array}\right)\right\}$, whose cardinality is at most $\left(\begin{array}{l}n \\ d\end{array}\right)=O\left(n^{d}\right)$; here $C>0$ is a constant that depends on $\alpha$ (and on $d$ ). In other words, we construct a $\lambda$-net with respect to the 1-dimensional set $X_{\ell} \subseteq \ell$, for $\lambda=\Theta\left(\epsilon^{d}\right)$. Our subsequent analysis in Section 5 effectively brings the density parameter $\lambda$ above $\epsilon^{d}$, which ultimately results in an even smaller $\lambda$-net $N_{\ell} \subset \ell$ of cardinality $O(1 / \lambda)=o\left(1 / \epsilon^{d}\right)$.

Notice that the existence of at least one vertical line $\ell$ for $K$, which would pierce many of the simplices $\left(\begin{array}{c}P_{K} \\ d\end{array}\right)$, is guaranteed by the Second Selection Theorem 2.1. To see this, we consider the vertical projection $P_{K}^{\perp}$ of $P_{K}=P \cap K$ (see Figure 3 (right)), apply the lemma to the complete $d$-uniform hypergraph $\left(\begin{array}{c}P_{K}^{\perp} \\ d\end{array}\right)$ (with $h=1$ ), and lift the resulting point $x$ back to a vertical line which now pierces a large fraction of the simplices of $\left(\begin{array}{c}P_{K} \\ d\end{array}\right) \cdot 9$

Unfortunately, the proposed choice of $\ell$ for our 1-dimensional reduction heavily depends on the convex set $K$ at hand. In fact, finding a small yet universal set of lines that would suit every $\epsilon$-heavy convex set $K$ is at least as hard as finding a weak $\epsilon$-net with respect to the $(d-1)$-dimensional projection of $P$.

To get around this problem, Chazelle et al. 22] cross the simplices of $\left(\begin{array}{c}P \\ d\end{array}\right)$ using a fairly large family $\mathcal{L}$ of vertical lines. Each line $\ell \in \mathcal{L}$ is assigned a canonical subset $\Pi_{\ell} \subseteq\left(\begin{array}{l}P \\ d\end{array}\right)$ of simplices (all of them crossed by $\ell$ ), and a $\lambda$-net $N_{\ell}$ is constructed with respect to its 1-dimensional set $X_{\ell}:=\left\{\operatorname{conv}(\tau) \cap \ell \mid \tau \in \Pi_{\ell}\right\}$, whose density $\lambda=\Omega^{*}\left(\epsilon^{d} n^{d} /\left|\Pi_{\ell}\right|\right)$ depends on $\left|\Pi_{\ell}\right|$. Despite the considerable size of $\mathcal{L}$, the ultimate cardinality of the net $\bigcup_{\ell \in \mathcal{L}} N_{\ell}$ is shown to be only $O^{*}\left(1 / \epsilon^{d}\right)$.

While the above method readily yields the bound $f_{d}(\epsilon)=O^{*}\left(1 / \epsilon^{d}\right)$, it is of little use to the more refined constructions in the sequel, which require a small universal line set $\mathcal{L}$.

The Multiple Selection Theorem. In Section 3 we show that a small yet universal set $\mathcal{L}$ of vertical lines can be found given that, for each convex set $K$ at hand, the points of the vertical projection $P_{K}^{\perp}$ of $P_{K}=P \cap K$ are reasonably well-spread within the projection $P^{\perp} \subseteq \mathbb{R}^{d-1}$ of the entire ground set $P$.

\footnotetext{
${ }^{9}$ The restricted variant of Theorem 2.1 for $h=1$ is known as the First Selection Theorem; see [42, Section 9] for a more comprehensive exposition. The reasons why we need the full generality of the Second Selection Theorem (and even more than that) will become clear already in Section 3
} 

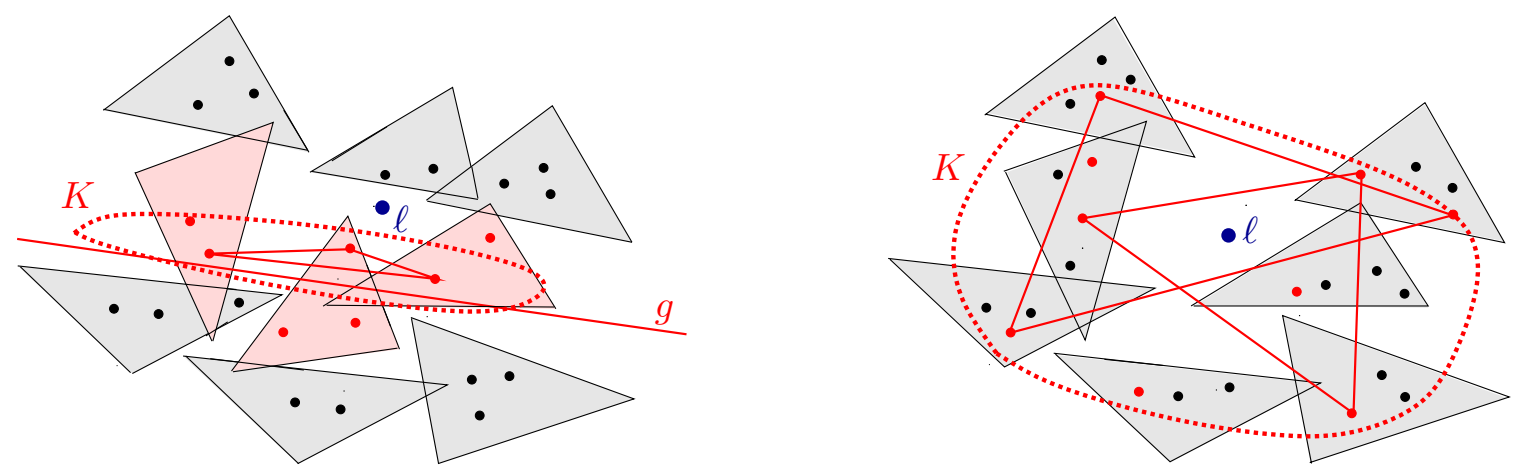

Figure 4: Using the vertical simplicial partition $\mathcal{P}(r)$ in $\mathbb{R}^{3}$ - view from above. Left: The set $K$ is narrow, so the points of $P_{K}$ project in the zone of the line $g \subseteq \mathbb{R}^{2}$ (red); the triangles of $\left(\begin{array}{c}P_{K} \\ 3\end{array}\right)$ are likely to be missed by our universal set of lines $\mathcal{L}$. Right: The set $K$ is spread, so many triangles in $\left(\begin{array}{c}P_{K} \\ 3\end{array}\right)$ are crossed by a line $\ell \in \mathcal{L}$.

To quantify the distribution of $P_{\bar{K}}^{\perp}$ within $P^{\perp}$, we fix a small parameter $r>0$, and use the Simplicial Partition Theorem 2.2 to obtain an $r$-partition $\mathcal{P}^{\perp}(r)=\left\{\left(P_{i}^{\perp}, \Delta_{i}\right) \mid 1 \leq i \leq r\right\}$ of $P^{\perp}$ in $\mathbb{R}^{d-1}$. We then lift $\mathcal{P}^{\perp}(r)$ back to the $d$-dimensional partition $\mathcal{P}(r)=\left\{\left(P_{i}, \Delta_{i}\right) \mid 1 \leq i \leq r\right\}$ of $P$ so that each subset $P_{i}$ is contained in the vertical prism above the respective simplex $\Delta_{i} \subset \mathbb{R}^{d-1}$, and satisfies $\left|P_{i}\right| \leq 2\lceil n / r\rceil$. In the sequel, we refer to the latter partition of $P$ as the vertical simplicial partition.

We say that our convex set $K$ is narrow if there is a $(d-2)$-plane $g$ in $\mathbb{R}^{d-1}$ whose zone with respect to $\mathcal{P}^{\perp}(r)$ encompasses a fixed fraction of the projected points of $P_{K}^{\perp}$; see Figure 4 (left). Otherwise, we say that $K$ is spread in $\mathcal{P}(r)$; see Figure 4 (right). Though the simplices in the narrow sets may prove hard for interception by few vertical lines, the bulk of their points are localized in only $O\left(r^{1-1 /(d-1)}\right)$ parts $P_{i}$ of the vertical partition $\mathcal{P}(r)$. In Section 3 we show that such sets can be easily pierced by a net whose cardinality is bounded by an essentially " $O\left(1 / \epsilon^{d-1}\right)$-type" recursive term $O\left(r \cdot f_{d}\left(\epsilon \cdot r^{1 /(d-1)}\right)\right) \cdot 10$

Specializing to the remaining convex sets $K$, which are more fairly spread in $\mathcal{P}(r)$, our Multiple Selection Theorem 3.3 yields the desired universal collection $\mathcal{L}$ of at most $r^{(d-1)^{2}}$ vertical lines, and with the following property: There exists a line $\ell \in \mathcal{L}$ that pierces a large fraction of the simplices of $\left(\begin{array}{c}P_{K} \\ d\end{array}\right)$ in each $\epsilon$-heavy convex set $K$.

Therefore, with the notable exception of the narrow sets, the Multiple Selection problem can be addressed by a combination of at most $r^{(d-1)^{2}} 1$-dimensional strong $\lambda$-nets $N_{\ell} \subset \ell$, for $\ell \in \mathcal{L}$ and $\lambda=\Omega\left(\epsilon^{d}\right)$. Most of our analysis (in Sections 4 and 50 is, therefore, devoted to bringing the effective density ratios $\lambda$ in these 1-dimensional problems significantly above $\epsilon^{d}$.

Hyperplanes are easy. It is well known that hypergraphs induced by hyperplane ranges have Vapnik-Chervonenkis dimension $d+1$ and, therefore, admit strong $\epsilon$-nets of cardinality $O\left(\frac{1}{\epsilon} \log \frac{1}{\epsilon}\right)$ 35. However, strong $\epsilon$-nets are no longer possible if the points of $P$ are slightly perturbed in a way that transforms many of the $\epsilon$-heavy hyperplanes into arbitrary thin, albeit strictly convex, sets. Instead, in Section 5 we construct a $d$-dimensional simplicial $t$-partition $\mathcal{Q}(s, t)$, for $\epsilon^{-d / 2} \leq$ $t=o\left(\epsilon^{-d}\right)$, and adopt a far broader (and, thereby, more useful) formulation. Informally, we will

\footnotetext{
${ }^{10}$ In the sequel, $r$ is chosen to be a very small, albeit fixed, degree of $1 / \epsilon$. Matoušek and Wagner 44 used a similar divide and conquer argument, albeit using a $d$-dimensional simplicial partition; the resulting recurrence $f_{d}(\epsilon)=r \cdot f_{d}\left(\epsilon \cdot r^{1 / d}\right)+r^{d^{2}}$ solved to $f_{d}(\epsilon)=O^{*}\left(1 / \epsilon^{d}\right)$
} 

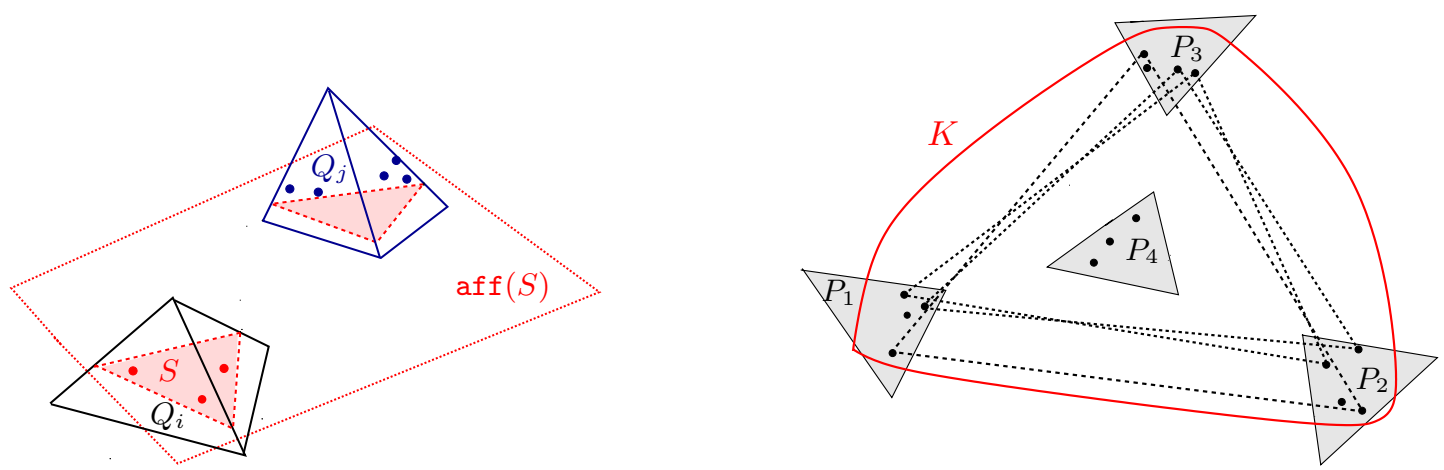

Figure 5: Left: Characterizing a tight set $K$ in $\mathbb{R}^{3}$ : For an average 3-set $S \in\left(\begin{array}{c}P_{K} \\ 3\end{array}\right)$, whose points come from the same part $Q_{i}$, the zone of aff $(S)$ encompasses a large fraction of the points in $P_{K}$. Right: The set $K$ is not vertically $\delta$-convex in $\mathbb{R}^{3}$ (view from above), hence, there is a characteristic 4 -tuple $\left(P_{1}, \ldots, P_{4}\right)$ in $\mathcal{P}(r)$ so that $P_{4}$ is surrounded by $P_{1}, P_{2}$, and $P_{3}$.

say that an $\epsilon$-heavy convex set $K$ is tight if for an average subset $S \in\left(\begin{array}{c}P_{K} \\ d\end{array}\right)$ of $d$ points that come from the same part $Q_{i} \in \mathcal{Q}(s, t)$, the zone of the hyperplane $H(S)=\operatorname{aff}(S)$ within $\mathcal{Q}(s, t)$ contains a large fraction of points of $P_{K}=P \cap K$; see Figure 5 (left).

A simple partitioning argument, that was previously used to bound the number of pointhyperplane incidences, yields a relatively small subset $\Pi_{\text {tight }} \subseteq\left(\begin{array}{l}P \\ d\end{array}\right)$, of cardinality $o\left(n^{d}\right)$, and with the following property: For any $\epsilon$-heavy tight convex set $K$, a large fraction of the $(d-1)$-simplices in $\left(\begin{array}{c}P_{K} \\ d\end{array}\right)$ belong to $\Pi_{\text {tight }}$. Therefore, specializing to the tight convex sets, the full hypergraph $\left(\begin{array}{c}P \\ d\end{array}\right)$ can be effectively replaced, for the purpose of our 1-dimensional reduction, by its smaller subset $\Pi_{\text {tight }} \subseteq\left(\begin{array}{c}P \\ d\end{array}\right)$.

Plugging this sparser set $\Pi_{\text {tight }}$, or rather its 1-dimensional $\ell$-intercepts $\{\operatorname{conv}(\tau) \cap \ell \mid \tau \in$ $\left.\Pi_{\text {tight }}\right\}$, into the 1-dimensional instance of each line $\ell \in \mathcal{L}$, will result in a much smaller net of cardinality $\tilde{O}\left(\left|\Pi_{\text {tight }}\right| / \epsilon^{d} n^{d}\right)=o\left(\epsilon^{-d}\right)$.

To dispatch the remaining convex sets, that cannot be easily "read-off" from (or at least sufficiently restricted by) their local $d$-subsets $S \in\left(\begin{array}{c}P_{K} \\ d\end{array}\right)$, in Section 5 we introduce several auxiliary nets, of cardinality $o\left(\epsilon^{-d}\right)$ each ${ }^{11}$ For example, the Strong Epsilon Net Theorem [35] can be used to get rid of the convex sets that contain a sufficiently heavy convex $(2 d)$-hedron. Furthermore, if a convex set $K$ is sufficiently thick, the points of $P_{K}$ can be effectively replaced by the vertices of their ambient simplices in $\mathcal{Q}(s, t)$, which again results in a better (i.e., larger) density parameter $\lambda$.

Characteristic $(d+1)$-tuples. Our main analysis in Section 5 requires that, for each convex set $K$ under consideration, the vertical partition $\mathcal{P}(r)$ contains $d+1$ subsets $P_{i_{1}}, \ldots, P_{i_{d+1}}$, with the following essential properties (where $0<\delta<1$ is a very small yet positive degree of $\epsilon$ ):

1. $\left|P_{i_{j}} \cap P_{K}\right|=\Omega(\delta \epsilon n / r)$, and

2. $P_{i_{d+1}}$ is surrounded by $P_{i_{1}}, \ldots, P_{i_{d}}$, in the following sense: For any choice of representatives $p_{j} \in P_{i_{j}}$ we have that $p_{d+1}^{\perp} \in\left(\operatorname{conv}\left(p_{1}, \ldots, p_{d}\right)\right)^{\perp}$. (In particular, the $(d+1)$-tuple $P_{i_{1}}^{\perp}, \ldots, P_{i_{d+1}}^{\perp}$ is homogeneous with respect to the order type; see, e.g., [30, 31].)

See Figure 5. In Section 5 we refer to $\left(P_{i_{1}}, \ldots, P_{i_{d+1}}\right)$ as the characteristic $(d+1)$-tuple of $K$.

\footnotetext{
${ }^{11}$ In what follows, such sets are called loose.
} 
Unfortunately, the desired characteristic $(d+1)$-tuple $\left(P_{i_{1}}, \ldots, P_{i_{d+1}}\right)$ may not exist for $K$ if its respective subset $P_{K}$ is in a vertically convex (or, rather, $\delta$-convex) position. Hence, we dedicate the entire Section 4 to piercing the sets $K$ that cut out vertically $\delta$-convex sub-sets $P_{K}$ within $P$. To that end, $\delta$ is set to be a very small, albeit fixed, degree of $\epsilon$. Informally, the simplices in the vicinity of the vertical silhouette $\partial\left(\operatorname{conv}\left(P_{K}\right)\right)^{\perp}$ of $\operatorname{conv}\left(P_{K}\right)$ may exhibit a very non-monotone behaviour while the entire set $K$ remains remarkably slim in $\mathbb{R}^{d}$ (and, therefore, difficult to pierce). To exploit the essentially $(d-2)$-dimensional structure of such subsets $P_{K}$, we invoke the more sensitive machinery of cuttings 23 in hyperplanes arrangements.

\subsection{Further essentials: Random sampling and arrangements of hyperplanes}

1. Random sampling. Let $X$ be a (finite) set of elements and $\mathcal{F} \subset 2^{X}$ be a set of hyperedges spanned by $X$. A strong $\epsilon$-net for the hypergraph $(X, \mathcal{F})$ is a subset $Y \subset X$ of elements so that $F \cap Y \neq \emptyset$ is satisfied for all hyperedges $F \in \mathcal{F}$ with $|F| \geq \epsilon n$.

Definition. Let $X$ be a set of $n$ elements, and $r>0$ be an integer. An $r$-sample of $X$ is a subset $Y \subset X$ of $r$ elements chosen at random from $X$, so that each such subset $Y \in\left(\begin{array}{c}X \\ r\end{array}\right)$ is selected with uniform probability $1 /\left(\begin{array}{l}n \\ r\end{array}\right)$.

The Epsilon-Net Theorem of Haussler and Welzl [35] states that any hypergraph $(X, \mathcal{F})$, as above, that is drawn from a so called range space of a bounded VC-dimension $D>0$, admits a strong $\epsilon$-net $Y$ of cardinality $r=O\left(\frac{D}{\epsilon} \log \frac{D}{\epsilon}\right)$. Moreover, such a net $Y$ can obtained, with probability at least $1 / 2$, by choosing an $r$-sample of $X$. In particular, this implies the following result.

Theorem 2.3. Let $P$ be a finite set of points in $\mathbb{R}^{d}$, then one can pierce all the $\epsilon$-heavy $d$ dimensional simplices with respect to $P$ using only $O\left(\frac{1}{\epsilon} \log \frac{1}{\epsilon}\right)$ points of $P$, where the constant of proportionality depends on the dimension $d$.

The following property of $r$-samples will prove useful in Sections 4 and 5 .

Lemma 2.4. There is a constant $C \geq 1$ so that the following property holds.

Let $A$ be a set of $m$ elements, and let $A=A_{1} \uplus A_{2} \uplus \ldots \uplus A_{r}$ be a partition of $A$ into $r \geq 1$ parts. Let $R$ be a random $r^{\prime}$-sample of $A$, so that $C r \leq r^{\prime} \leq m-\frac{m}{100 r}$. Then, with probability at least $1 / 2$, the parts $A_{i}$ that intersect $R$, encompass at least $m / 3$ elements of $A$.

Proof. Clearly, at most $m / 100$ elements of $A$ belong to the partition sets $A_{i}$ with $\left|A_{i}\right| \leq m /(100 r)$. Let $A^{\prime}:=\bigcup\left\{A_{i}|1 \leq i \leq r,| A_{i} \mid m /(100 r)\right\}$. Then we have $\left|A^{\prime}\right| \geq m-r \cdot m /(100 r) \geq 99 r / 100$. Fix any $x \in A^{\prime}$ with the ambient set $A_{i(x)}$, so that $1 \leq i(x) \leq r$. Since $\left|A_{i(x)}\right|>m /(100 r)$, the probability $\mathbf{p}_{x}$ that $A_{i(x)}$ is missed by the sample $R$ satisfies

$$
\mathbf{p}_{x} \leq\left(\begin{array}{c}
m-m /(100 r) \\
r^{\prime}
\end{array}\right) /\left(\begin{array}{l}
m \\
r^{\prime}
\end{array}\right) \leq\left(1-\frac{1}{100 r}\right)^{C r} .
$$

A suitable choice of $C>1$ guarantees that the right hand side of this bound is at most $1 / 100$ (or any other constant). Hence, the expected number of elements in the union $\bigcup_{x \in R} A_{i(x)}$ of the parts that are marked by $R$, is

$$
\sum_{x \in A^{\prime}} \mathbf{p}_{x} \geq 0.99 \cdot 99 m / 100
$$

Now the lemma follows by Markov's inequality. 
Lemma 2.5. For any dimension $d \geq 2$ there is a constant $C_{\text {samp }}>0$ with the following property: Let $A$ be a vertically $\delta$-convex $m$-point set in $\mathbb{R}^{d}$, and let $R \subseteq A$ be an $r$-sample of $A$, so that $r \leq m$ and $\delta \leq C_{\text {samp }} / r^{d+1}$. Then $R$ is vertically convex with probability at least $99 / 100$.

Proof. We can assume that $r \geq d+1$, or else the vertical $\delta$-convexity holds trivially for the empty set $\left(\begin{array}{c}R \\ d+1\end{array}\right)$. Let $B \in\left(\begin{array}{c}A \\ d+1\end{array}\right)$ be a $(d+1)$-subset that does not have a convex projection. Notice that $B$ arises in our $r$-sample $R \subset A$, as a subset in $\left(\begin{array}{c}R \\ d+1\end{array}\right)$, with probability $\mathbf{p}:=\frac{\left(\begin{array}{c}m-d-1 \\ r-d-1\end{array}\right)}{\left(\begin{array}{c}m \\ r\end{array}\right)}=\Theta\left(\frac{r^{d+1}}{m^{d+1}}\right)$.

Hence, the expected number of such $(d+1)$-subsets $B \in\left(\begin{array}{c}A \\ d+1\end{array}\right)$ is at most

$$
\delta\left(\begin{array}{c}
m \\
d+1
\end{array}\right) \mathbf{p}=O\left(\delta\left(\begin{array}{c}
m \\
d+1
\end{array}\right)\left(\frac{r}{m}\right)^{d+1}\right) .
$$

A suitable choice of $C_{\text {samp }}$ guarantees that the right-hand side of (1) is always smaller than 1/100. In particular, this implies that, with probability at least $99 / 100$, the all $(d+1)$-subsets $\left(\begin{array}{c}A \\ d+1\end{array}\right)$ are vertically convex.

2. Arrangements of hyperplanes in $\mathbb{R}^{d}$. For any $d$-point set (or a $(d-1)$-dimensional simplex) $S$ in a general position in $\mathbb{R}^{d}$, let $H(S)$ denote the hyperplane aff $(S)$ supported by $S$. Given a set of hyperedges $\Pi \subset\left(\begin{array}{l}P \\ d\end{array}\right)$, let $\mathcal{H}(\Pi):=\{H(S) \mid S \in \Pi\}$ be the set of all the hyperplanes spanned by the hyperedges of $\Pi$.

Our divide-and-conquer argument in Section 4 uses cells in an arrangement of hyperplanes that are sampled at random from among the hyperplanes $H(\tau)$ that support the simplices $\tau$ of our restriction hypergraph $(P, \Pi)$.

Definition. Any finite family $\mathcal{H}$ of $m$ hyperplanes in $\mathbb{R}^{d}$ induces the arrangement $\mathcal{A}(\mathcal{H})$ - the partition of $\mathbb{R}^{d}$ into open faces. The $d$-dimensional faces of $\mathcal{A}(\mathcal{H})$ are called cells, and they are maximal connected regions of $\mathbb{R}^{d} \backslash(\bigcup \mathcal{H})$. Each cell is a convex polyhedron whose boundary is comprised of $k$-dimensional faces of $\mathcal{A}(\mathcal{H})$, for $0 \leq k \leq d-1$; each of these faces is a $k$ dimensional polyhedron which lies within a $k$-dimensional plane - an intersection of some $d-k$ of the hyperplanes of $\mathcal{H}$. Specifically, the $(d-1)$-dimensional faces of $\mathcal{A}(\mathcal{H})$ are $(d-1)$-dimensional polyhedral portions of the hyperplanes of $\mathcal{H}$. The 0 -dimensional faces are called vertices, and each of them is an intersection of some $d$ of the hyperplanes in $\mathcal{H}$.

It is known that an arrangement $\mathcal{A}(\mathcal{H})$ of $n$ hyperplanes encompasses a total of $O\left(n^{d}\right)$ faces of all dimensions (and this bound is tight for $\mathcal{H}$ in a general position).

The complexity $\varpi(\Delta)$ of a polyhedral cell $\Delta$ of $\mathcal{A}(\mathcal{H})$ is the total number of faces of all dimensions between 0 and $d-1$ that lie on its boundary. See, e.g., [42, Section 6] for a more comprehensive background on hyperplane arrangements.

One can get rid of unbounded faces in $\mathcal{A}(\mathcal{H})$ by augmenting $\mathcal{H}$ with a pair of imaginary hyperplanes that are orthogonal to the $i$-th axis, $H_{i}^{+}:=\left\{X_{i}=\infty\right\}, H_{i}^{-}:=\left\{X_{i}=-\infty\right\}$, for each $1 \leq i \leq d$. With this adjustment, every original cell in $\mathcal{A}(\mathcal{H})$ now corresponds to exactly one polytopal cell in the augmented arrangement $\mathcal{A}(\mathcal{H})$. In what follows, we restrict our analysis to these latter cells.

Theorem 2.6. Let $\mathcal{H}$ be a family of $m$ hyperplanes in $\mathbb{R}^{d}$, and $0<r \leq m$ integer. Then, with probability at least $1 / 2$, an r-sample $\mathcal{R} \in\left(\begin{array}{c}\mathcal{H} \\ r\end{array}\right)$ of $\mathcal{H}$ crosses every open segment in $\mathbb{R}^{d}$ that is intersected by at least $C(\mathrm{~m} / \mathrm{r}) \log r$ hyperplanes of $\mathcal{H}$. Here $C>0$ is a sufficiently large constant that does not depend on $m$ or $r$. 
The proof of Theorem 2.6 can be found, e.g., in [21. It is established by applying the Epsilon Net Theorem to the range space in which every vertex set is a finite family $\mathcal{H}$ of hyperplanes in $\mathbb{R}^{d}$, and each hyperedge consists of all the hyperplanes in $\mathcal{H}$ that are crossed by some segment in $\mathbb{R}^{d}$.

3. The bottom-vertex triangulation. Every polytopal cell $\Delta$ of $\mathcal{A}(\mathcal{H})$ can be decomposed into a collection $\mathcal{D}_{\Delta}$ of interior-disjoint $d$-dimensional simplices that share the lowermost boundary vertex $v$ of $\Delta 12$ Each of these simplices is of the form $\tau=\operatorname{conv}(\{v\} \cup \kappa)$ where $\kappa$ is a $(d-1)$-dimensional simplex that is not adjacent to $v$, and arises in a recursive triangulation of some $(d-1)$-dimensional face on the boundary of $\Delta$. Let $\mathcal{D}(\mathcal{H})$ denote the resulting collection of interior-disjoint simplices $\bigcup_{\Delta \in \mathcal{A}(\mathcal{H})} \mathcal{D}_{\Delta}$ that comprise the bottom-vertex triangulations of all the $d$-cells of $\mathcal{A}(\mathcal{H})$.

An easy consequence of Theorem 2.6 is that, with probability at least $1 / 2$, every cell of $\mathcal{D}(\mathcal{R})$ is crossed by at most $(d+1) C(m / r) \log r$ hyperplanes of $\mathcal{H}$; in other words, it serves as an $\left(\frac{(d+1) C \log r}{r}\right)$-cutting of $\mathcal{H}$ [42]. (This is because every hyperplane of $\mathcal{H}$ that crosses a simplex $\tau$ of $\mathcal{D}(\mathcal{R})$ must also cross at least $d$ of the $\left(\begin{array}{c}d+1 \\ 2\end{array}\right)$ 1-dimensional faces, or edges, of $\tau$.)

Definition. Let $\mathcal{C}$ be a family of polytopal cells in $\mathbb{R}^{d}$ (e.g., faces in the above arrangement $\mathcal{A}(\mathcal{H})$, or the $d$-dimensional simplices of its refinement $\mathcal{D}(\mathcal{H})$ ). The zone of any set $X \subset \mathbb{R}^{d}$ in $\mathcal{C}$ is the subset of all the cells in $\mathcal{C}$ that intersect $X$.

4. The complexity of many cells. The following elementrary bound easily follows through induction on the ambient dimension $d$; see, e.g., [10, 52].

Theorem 2.7. Let $\mathcal{H}$ be a collection of $n$ hyperplanes in $\mathbb{R}^{d}$, and $K \subset \mathbb{R}^{d}$ a convex set. Then the boundary $\partial K$ of $K$ intersects $O\left(n^{d-1}\right)$ cells in the arrangement $\mathcal{A}(\mathcal{H})$.

Note that for any collection $\mathcal{H}$ of $n$ hyperplanes, the overall complexity of all the cells in their arrangement $\mathcal{A}(\mathcal{H})$ is $O\left(n^{d}\right)$. (This is because, after perturbing $\mathcal{H}$ into a general position, every $k$-dimensional face is adjacent to at most $2^{d-k}$ cells.) However, if the cardinality of $\mathcal{C}$ is much smaller than $n^{d}$, then the overall complexity $\varpi(\mathcal{C})=\sum_{\Delta \in \mathcal{C}} \varpi(\Delta)$ is $o\left(n^{d}\right)$. Let us quantify this phenomenon in the terms of $n$ and $m$.

Theorem 2.8 (Aronov, Matousek, Sharir 1994; Aronov, Sharir 2004). Let $\mathcal{H}$ be a collection of $n$ hyperplanes in $\mathbb{R}^{d}$. Then for any collection $\mathcal{C}$ of $m$ cells in $\mathcal{A}(\mathcal{H})$, their overall complexity $\varpi(\mathcal{C})$ is $O\left(m^{1 / 2} n^{d / 2} \log ^{\eta_{d}} n\right)$, where $\eta_{d}=(\lfloor d / 2\rfloor-1) / 2$.

\subsection{Proof of Theorem 1.1: the recursive framework and roadmap}

We refine the notation of Section 1 and lay down the formal framework in which our analysis is cast.

Definition. We say that $N \subset \mathbb{R}^{d}$ is a weak $\epsilon$-net for a family $\mathcal{K}$ of convex sets in $\mathbb{R}^{d}$ if it pierces every $\epsilon$-heavy set in $\mathcal{K}$. (In particular, every weak $\epsilon$-net with respect to $P$ is also a weak $\epsilon$-net for any subfamily $\mathcal{K}$ of convex sets in $\mathbb{R}^{d}$.)

For a finite point set $P$ in $\mathbb{R}^{d}$ and $\epsilon>0$, let $\mathcal{K}(P, \epsilon)$ denote the family of all the $\epsilon$-heavy convex sets with respect to $P$.

If the parameter $\epsilon$ is fixed, we can assume that each set in the family $\mathcal{K}$ is $\epsilon$-heavy (that is $\mathcal{K} \subseteq \mathcal{K}(P, \epsilon))$, so $N$ is simply a point transversal to $\mathcal{K}$.

\footnotetext{
${ }^{12}$ The vertex $v$ is unique given that no hyperplane in $\mathcal{H}$ is horizontal.
} 
Notice that the previous constructions [5, 22, 44] employed recurrence schemes in which every problem instance $(P, \epsilon)$ was defined over a finite point set $P$, and sought to pierce each $\epsilon$-heavy convex set $K \in \mathcal{K}(P, \epsilon)$ using the smallest possible number of points. This goal was achieved in a divide-and-conquer fashion, by tackling a number of simpler sub-instances $\left(P^{\prime}, \epsilon^{\prime}\right)$ with smaller point sets $P^{\prime} \subset P$ and larger parameters $\epsilon^{\prime}>\epsilon$.

However, the discussion in Section 2.2 implies, by the means of our 1-dimensional reduction, the existence of more favourable bounds if the simplices that are induced by the convex sets, are restricted to a sparser subset of $\left(\begin{array}{c}P \\ d\end{array}\right)$, of cardinality $o\left(n^{d}\right)$. Therefore, to amplify our $o\left(\epsilon^{d}\right)$ bounds on $f_{d}(\epsilon)$, we employ a somewhat more refined recursive framework: the convex sets $K$ are now restricted not only in the terms of the underlying point set $P$, but also in the terms of a certain collection $\Pi$ of $(d-1)$-dimensional simplices $\Pi$ which encompasses a large fraction of the $(d-1)$ simplices determined by $P \cap K$. Thus, our recurrence can advance not only by increasing the parameter $\epsilon$, but also by restricting the convex sets to "include" many $(d-1)$-simplices of the (typically, sparse) subset $\Pi \subset\left(\begin{array}{l}P \\ d\end{array}\right)$.

Definition. Let $\Pi \subset\left(\begin{array}{l}P \\ d\end{array}\right)$ be a subset of $d$-hyperedges spanned by the underlying $n$-point set $P$. Let $\sigma>0$. We say that a convex set $K$ is $(\epsilon, \sigma)$-restricted to the $d$-uniform hypergraph $(P, \Pi)$ if $P \cap K$ contains a subset $P_{K}$ of $\lceil\epsilon n\rceil$ points so that the induced subset of hyperedges $\Pi_{K}=\left(\begin{array}{c}P_{K} \\ d\end{array}\right) \cap \Pi$ contains at least $\sigma\left(\begin{array}{c}\epsilon n \\ d\end{array}\right)$ hyperedges. (In particular, each $(\epsilon, \sigma)$-restricted set $K$ must be $\epsilon$-heavy with respect to $P$.)

Notice that the choice of $P_{K}$ as above, may not be unique, for $K$ may enclose additional points of $P$. To simplify the exposition, in the sequel we select a unique witness set $P_{K}$ for every convex set $K$ that is $(\epsilon, \sigma)$-restricted to $(P, \Pi)$.

At each recursive step we construct a weak $\epsilon$-net $N$ that pierces a certain family $\mathcal{K}=\mathcal{K}(P, \Pi, \epsilon, \sigma)$ of convex sets which is determined by $\epsilon>0$, a finite point set $P \subset \mathbb{R}^{d}$, a set of hyperedges $\Pi \subseteq\left(\begin{array}{c}P \\ d\end{array}\right)$, and a threshold $0<\sigma \leq 1$. This family $\mathcal{K}$ consists of all the convex sets $K$ that are $(\epsilon, \sigma)$-restricted to $(P, \Pi)$.

In what follows, we refer to $(P, \Pi)$ (or simply to $\Pi$ ) as the restriction hypergraph, and to $\sigma$ as the restriction threshold of the recursive instance.

The topmost instance of the recurrence involves $\Pi=\left(\begin{array}{l}P \\ d\end{array}\right)$ and $\sigma=1$. Each subsequent instance $\mathcal{K}^{\prime}=\mathcal{K}\left(P^{\prime}, \Pi^{\prime}, \epsilon^{\prime}, \sigma^{\prime}\right)$ involves a larger $\epsilon^{\prime}$ and/or a much sparser restriction hypergraph $\left(P^{\prime}, \Pi^{\prime}\right)$. Each such increase in $\epsilon$ or decrease in the density $\rho:=|\Pi| /\left(\begin{array}{l}n \\ d\end{array}\right)$ is accompanied only by a comparatively mild decrease in the restriction threshold $\sigma$, which is bounded from below by a certain positive constant throughout the recurrence. 13

The above recurrence bottoms out when either $\epsilon$ surpasses a certain (suitably small) constant $0<\epsilon_{0}<1$, or the density $\rho$ of the restriction hypergraph falls below $\epsilon^{d}$. In the former case we can use the $O^{*}\left(\left(1 / \epsilon_{0}\right)^{d}\right)=O(1)$ bound of Chazelle et al. [22], and in the latter we resort to a much simpler sub-recurrence of Theorem 3.7 below.

Deriving a recurrence for $f(\epsilon)$. In the course of our analysis we stick with the following notation. We use $f(\epsilon, \rho, \sigma)$ to denote the smallest number $f$ so that for any finite point set $P$ in $\mathbb{R}^{d}$, and any subset $\Pi \subset\left(\begin{array}{l}P \\ d\end{array}\right)$ of density $\rho \leq|\Pi| /\left(\begin{array}{l}n \\ d\end{array}\right)$, there is a point transversal of size $f$ to $\mathcal{K}(P, \Pi, \epsilon, \sigma)$. We set $f(\epsilon, \rho, \sigma)=1$ whenever $\epsilon \geq 1$. Since the underlying dimension $d$ is fixed, for the sake of brevity we

\footnotetext{
${ }^{13}$ The preceding discussion in Section 2.2 which we formalize in Theorem 3.7 yields a net of cardinality $o\left(1 / \epsilon^{d}\right)$ once the density $\rho$ falls substantially below 1 (given that the restriction threshold $\sigma$ remains close to 1 ). Hence, our further recurrence over $\Pi$ is used to merely amplify this gain.
} 
use $f(\epsilon)$ to denote the quantity $f_{d}(\epsilon)=f(\epsilon, 1,1)$, and note that the trivial bound $f(\epsilon, \rho, \sigma) \leq f(\epsilon)$ always holds.

In the sequel, we bound the quantity $f(\epsilon, \rho, \sigma)$ by a recursive expression of the general form

$$
f(\epsilon, \rho / h, \sigma / 2)+\sum_{i=1}^{k} r_{i} \cdot f\left(\epsilon \cdot r_{i}^{a_{i}}\right)+O\left(1 / \epsilon^{d-1 / 2+\gamma^{\prime}}\right),
$$

where $k$ is a constant, $a_{i} \geq 2 /(2 d-1)-\gamma^{\prime}$ for all $1 \leq i \leq k$, the respective parameters $r_{i}$ and $h \lll r_{i}$ are arbitrary small (albeit fixed) degrees of $1 / \epsilon$, and $\gamma^{\prime}>0$ is an arbitrary small constant; in particular, this bound will hold for $f(\epsilon)=f(\epsilon, 1,1)$.

Since the recurrence bottoms out for $\rho=O\left(\epsilon^{d}\right)$, one can easily get rid of the first term in (2). The standard (and fairly general) substitution methodology (see, e.g., 44, 54] and [55, Section 7.3.2]) shows that such recurrences solve to $f(\epsilon)=O\left(\frac{1}{\epsilon^{d-1 / 2+\gamma}}\right)$, for any constant $\gamma>0$.

To obtain the desired recurrence for $f(\epsilon, \rho, \sigma)$, we fix an arbitrary $n$-point set $P$ in a general position, and a restriction hypergraph $\Pi \subseteq\left(\begin{array}{l}P \\ d\end{array}\right)$ so that $|\Pi| /\left(\begin{array}{l}n \\ d\end{array}\right) \leq \rho$. In the sequel, we construct a point transversal $N$ for the resulting family $\mathcal{K}(P, \Pi, \epsilon, \sigma)$ and show that the cardinality of $N$ is bounded by a recursive expression of the general form (2). Clearly, we can assume with no loss of generality that $n \geq 1 / \epsilon^{d-1 / 2}$ (or we can set $N:=P$ ).

As mentioned in Section 2.2, the vertically $\delta$-convex sets pose a unique challenge due to the exceptional properties of the simplices that are adjacent to the vertical silhouette conv $\left(P^{\perp}\right)$. To this end, we fix an integer parameter $r>0$ that proportional to a very small, albeit fixed, degree of $1 / \epsilon$ (that satisfies $r \lll 1 / \epsilon)$, and set

$$
\delta:=C_{\mathrm{vcon}} / r^{d(d+1)+1},
$$

where $C_{\mathrm{vcon}}>0$ is a suitable constant to be determined in the end of Section 4.

Let $\mathcal{K}_{\mathrm{vcon}}$ denote the sub-family comprised of all the convex sets $K \in \mathcal{K}(P, \Pi, \epsilon, \sigma)$ that are vertically $\delta$-convex, and denote $\mathcal{K}_{\text {surr }}$ denote the complementary sub-family $\mathcal{K}(P, \Pi, \epsilon, \sigma) \backslash \mathcal{K}_{\text {vcon }}$. In what follows, we derive a separate recurrence, of the general form (4), for each of these families $\mathcal{K}_{\text {vcon }}$ and $\mathcal{K}_{\text {surr }}$.

Proof of Theorem 1.1 - the roadmap. In Section 3 we establish the basic reduction of the weak $\epsilon$-net problem to a small number of 1-dimensional nets.

In Sections 4 and 5 we construct small-size nets $N_{\text {vcon }}$ and $N_{\text {surr }}$ for the above families $\mathcal{K}_{\text {vcon }} \subseteq$ $\mathcal{K}(P, \Pi, \epsilon, \sigma)$ and $\mathcal{K}_{\text {surr }} \subseteq \mathcal{K}(P, \Pi, \epsilon, \sigma)$, respectively. Notice that both arguments build on the results of Section 3 .

In Section 6 we combine the upper bounds on the cardinalities of the net $N_{\text {vcon }}$ and $N_{\text {surr }}$ to establish the desired recurrence for the quantity $f(\epsilon)$ and, thereby, complete the proof of Theorem 1.1. In addition, we conclude the paper with several closing remarks.

\section{Crossing convex sets by vertical lines}

\subsection{The vertical simplicial partition}

As noted in Section 2.2, we seek to pierce the convex sets $K \in \mathcal{K}(P, \epsilon)$ using relatively few vertical lines. However, this is possible only if the points of the vertical projection $(P \cap K)^{\perp}$ are reasonably well-distributed within the projected set $P^{\perp}$, whereas the other sets of $\mathcal{K}(P, \epsilon)$ may require a separate treatment due to their relatively "skinny" vertical projections. 
To quantify the above phenomenon, we vertically project the underlying point set $P$ onto the copy of $\mathbb{R}^{d-1}$ that is spanned by the first $d-1$ axis, and apply the Simplicial Partition Theorem 2.2 to the projected set $P^{\perp} \subset \mathbb{R}^{d}$, and with a very small partition parameter $r \ll 1 / \epsilon$ which will be determined in the sequel.

Definition. Let $P$ be an $n$-point set in a general position in $\mathbb{R}^{d}$. We say that $\left\{\left(P_{i}, \Delta_{i}\right) \mid 1 \leq i \leq r\right\}$ is a vertical simplicial $r$-partition (or, shortly, a vertical simplical partition, if the parameter $r$ is clear from the context) if $\left\{\left(P_{i}^{\perp}, \Delta_{i}\right) \mid 1 \leq i \leq r\right\}$ is a simplicial $r$-partition of the projected set $P^{\perp}$ in $\mathbb{R}^{d-1}$ that meets the criteria of Theorem 2.2. (In particular, each $\Delta_{i}$ is a $(d-1)$-dimensional simplex in $\mathbb{R}^{d-1}$, and each subset $P_{i}$ is contained in the vertical prism that is raised over $\Delta_{i}$.)

For each $1 \leq i \leq r$, and each point $p \in P_{i}$, we refer to $\Delta_{i}$ as the ambient simplex of $p$.

Definition. We say that a convex set $K \subseteq \mathbb{R}^{d}$ is $\epsilon^{\prime}$-narrow with respect to the vertical simplicial partition $\mathcal{P}=\left\{\left(P_{i}, \Delta_{i}\right) \mid 1 \leq i \leq r\right\}$ if there exists a $(d-2)$-plane $g$ within $\mathbb{R}^{d-1}$ whose zone within the respective $(d-1)$-dimensional partition $\mathcal{P}^{\perp}=\left\{\left(P_{i}^{\perp}, \Delta_{i}\right) \mid 1 \leq i \leq r\right\}$ encompasses at least $\epsilon^{\prime} n$ of the projected points of $(P \cap K)^{\perp}$. See Figure 4 (left).

If the convex set $K \subseteq \mathbb{R}^{d}$ is not $\epsilon^{\prime}$-narrow with respect to a vertical simplicial partition $\mathcal{P}$, we say that $K$ is $\epsilon^{\prime}$-spread in $\mathcal{P}$. (See Figure 4 (right).)

Remark. In particular, every convex set outside $\mathcal{K}(P, \epsilon)$ is $\epsilon$-spread. However, most applications of the theorem involve convex sets $K$ that are at least $\epsilon$-heavy with respect to the underlying point set.

\subsection{Piercing narrow convex sets}

The following lemma yields a favourable recurrence for all the $\tilde{\Omega}(\epsilon)$-narrow convex sets.

Lemma 3.1. Let $P$ be a finite point set in $\mathbb{R}^{d}$, and $\epsilon^{\prime}>0$. Let $r>0$ be an integer, and $\mathcal{P}(r)$ be a vertical simplicial $r$-partition of $P$ as described in Section 3.1. Then there exists a set $N\left(\epsilon^{\prime}, r\right) \subset \mathbb{R}^{d}$, of cardinality $O\left(r \cdot f\left(\epsilon^{\prime} \cdot r^{1 /(d-1)}\right)\right)$, that pierces every convex set $K$ that is $\epsilon^{\prime}$-narrow with respect to $\mathcal{P} 14$

Proof. For every $1 \leq i \leq r$ we recursively construct a net $N_{i}$ for the local problem $\mathcal{K}\left(P_{i}, \tilde{\epsilon}\right)$, where

$$
\tilde{\epsilon}:=C \cdot \epsilon^{\prime} r^{1 /(d-1)}=\Theta\left(\frac{\epsilon^{\prime} n /\left(r^{1-1 /(d-1)}\right)}{n / r}\right),
$$

and $C>0$ is a suitably small fixed constant that does not depend on $\epsilon^{\prime}$ or $r$ (but may depend on $d$. Notice that each $N_{i}$ is a $d$-dimensional net of cardinality $\left|N_{i}\right| \leq f(\tilde{\epsilon})$.

It suffices to show that $N\left(\epsilon^{\prime}, r\right):=\bigcup_{1 \leq i \leq r} N_{i}$ pierces all the $\epsilon^{\prime}$-narrow convex sets. Indeed, let $K$ be such a narrow convex set with respect to $\mathcal{P}$, and $g$ be the witness $(d-2)$-plane within $\mathbb{R}^{d-1}$. By definition, at least $\epsilon^{\prime} n$ points of $P \cap K$ belong to such sets $P_{i}$ whose ambient $(d-1)$-simplices $\Delta_{i} \subset \mathbb{R}^{d-1}$ are crossed by $g$. Since the number of the latter simplices is $O\left(r^{1-1 /(d-1)}\right)$ (via Theorem 2.2 , by the pigeonhole principle there must be such a set $P_{i}$ that contains $\Omega\left(\epsilon^{\prime} n /\left(r^{1-1 /(d-1)}\right)\right)$ of the points of $P \cap K$. A suitable choice of the constant $C>0$ guarantees that $K$ is pierced by the respective net $N_{i}$.

\footnotetext{
${ }^{14}$ For the sake of brevity, in the sequel we routinely omit the constant multiplicative factors within the recursive terms of the general form $a \cdot f(\epsilon \cdot b)$, provided that these factors are much larger than $1 / b$ and $1 / a$.
} 


\subsection{A refined Selection Theorem}

Our proof of the Multiple Selection Theorem 3.3 uses a refined (and more explicit) variant of the Second Selection Theorem 2.1, where some vertices of the geometric graph $(P, \Pi)$ may overlap in $\mathbb{R}^{d}$.

Definition. For any abstract $(d+1)$-uniform hypergraph $(V, E)$, where $E \subseteq\left(\begin{array}{c}V \\ d+1\end{array}\right)$, we identify its embeddings in $\mathbb{R}^{d}$ with functions $\chi: V \rightarrow \mathbb{R}^{d}$. Specifically, we define $\chi(V):=\{\chi(v) \mid v \in V\}$, $\chi(f)=\left\{\chi\left(v_{1}\right), \ldots, \chi\left(v_{d+1}\right)\right\}$ for any $f=\left\{v_{1}, \ldots, v_{d+1}\right\} \in E$, and $\chi(E):=\{\chi(f) \mid f \in E\}$.

We say that such an embedding $\chi: V \rightarrow \mathbb{R}^{d}$ of $(V, E)$ is $C$-conforming (for $C \geq 1$ ) if the set $\chi(V)$ is in general position, and we have that $\left|\chi^{-1}(x)\right| \leq C$ for any $x \in \mathbb{R}^{d}$. (In other words, at most $C$ vertices of $V$ are mapped by $\chi$ to any given point $x \in \mathbb{R}^{d}$.)

Theorem 3.2. For any dimension $d \geq 1$ there exist constants $b=2 d+2, C_{d}>0, D_{d}>0$, and $\beta_{d} \geq t^{(d+1)}$ with the following properties:

Let $(V, E)$ be a $(d+1)$-uniform hypergraph so that $|V|=n$ and $E \geq h\left(\begin{array}{c}n \\ d+1\end{array}\right)$, and $\chi: V \rightarrow \mathbb{R}^{d}$ be a $\left(C_{d} h^{\beta_{d}} n\right)$-conforming embedding of $(V, E)$ in $\mathbb{R}^{d}$. Then there is a point $x_{0} \in \mathbb{R}^{d}$, and a subset $E_{0} \subseteq E$ of $\Omega\left(h^{\beta_{d}}\left(\begin{array}{c}n \\ d+1\end{array}\right)\right)$ edges, so that $x_{0} \in \operatorname{conv}(\chi(f))$ for each $f \in E_{0}$. Furthermore, if $h \geq D_{d} n^{-1 / t^{d}}$, then there exist d pairwise disjoint $(d+1)$-sets $A_{1}, A_{2}, \ldots, A_{d} \in \chi(E)$, with subsets $B_{i} \in\left(\begin{array}{c}A_{i} \\ d\end{array}\right)$, so that $x_{0}=\bigcap_{i=1}^{d} \operatorname{aff}\left(B_{i}\right)$.

Though Theorem 3.2 is derived nearly verbatim via the original proof of the Second Selection Theorem 2.1 by Alon, Bárány, Füredi, and Kleitman [1, Section 6] (using roughly the same combination of the Erdős-Simonovits Theorem [27] and an improved variant [14] of the Colorful Tverberg Theorem [59]), we spell out the full details in Appendix A.

\subsection{Multiple Selection: Piercing many $(d-1)$-dimensional simplices with few vertical lines}

In this section we construct a small canonical set $\mathcal{L}$ of vertical lines so that for any $\tilde{O}(\epsilon)$-spread convex set $K \in \mathcal{K}(P, \Pi, \epsilon, \sigma)$, roughly $\Omega\left(\epsilon^{d} n^{d}\right)$ simplices of $\Pi_{K} \subseteq\left(\begin{array}{c}P_{K} \\ d\end{array}\right)$ can be crossed by a single line of $\mathcal{L}$. To that end, we extend the Second Selection Theorem 2.1 to the $d$-uniform simplicial hypergraphs in $\mathbb{R}^{d-1}$ that are induced by the vertical projections of unknown convex sets $K \in$ $\mathcal{K}(P, \epsilon)$.

In view of the subsequent applications, the main result of this section is formulated in the terms of vertical line transversals to $(d-1)$-dimensional simplices of $\left(\begin{array}{c}P_{K} \\ d\end{array}\right)$.

Definition. We say that a point $x$ in $\mathbb{R}^{d-1}$ is surrounded by $d$ sets $S_{1}, \ldots, S_{d} \subseteq \mathbb{R}^{d-1}$ if for every choice $x_{1} \in S_{1}, x_{2} \in S_{2}, \ldots, x_{d} \in S_{d}$, the point $x$ lies in $\operatorname{conv}\left(p_{1}, \ldots, p_{d}\right){ }^{15}$

We say that a line $\ell$ in $\mathbb{R}^{d}$ is surrounded by the $d$ sets $S_{1}, \ldots, S_{d} \subseteq \mathbb{R}^{d-1}$ if the vertical intercept of $\ell$ in $\mathbb{R}^{d-1}$ is surrounded by these sets.

Note that, if $\ell$ is surrounded by simplices $\Delta_{i_{1}}, \ldots, \Delta_{i_{d}} \subset \mathbb{R}^{d-1}$ in the vertical $r$-partition $\mathcal{P}(r)$ of Section 3.1, for $1 \leq i_{1} \neq i_{2} \neq \ldots \neq i_{d} \leq r$, then it pierces every simplex $\operatorname{conv}\left(p_{1}, \ldots, p_{d}\right)$ so that $p_{j} \in P_{i_{j}}$.

\footnotetext{
${ }^{15}$ In particular, the $d$-tuple $\left(S_{1}, \ldots, S_{d}\right)$ is homogeneous with respect to $x$ in the notation of Fox, Gromov, Lafforgue, Naor, and Pach [30.
} 


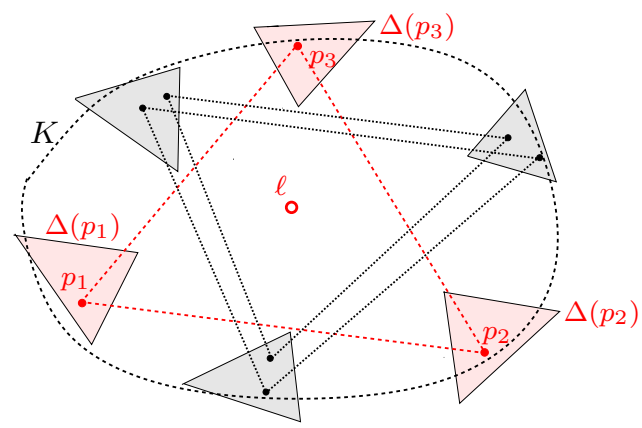

Figure 6: Case 1 of the Multiple Selection Theorem in dimension $d=3$ (view from above). The vertical line $\ell \in \mathcal{L}(r)$ pierces a subset $\Pi_{K}(\ell)$ of $\Omega\left(\sigma^{\beta_{2}}\left(\begin{array}{c}\lceil\epsilon n\rceil \\ 3\end{array}\right)\right)$ simplices $\left\{p_{1}, p_{2}, p_{3}\right\} \in \Pi_{K}$ such that $\ell$ is surrounded by the respective ambient simplices $\Delta\left(p_{1}\right), \Delta\left(p_{2}\right), \Delta\left(p_{3}\right) \subset \mathbb{R}^{2}$, of their vertices $p_{1}, p_{2}$, and $p_{3}$.

Theorem 3.3. Let $P$ be a ground n-point set in $\mathbb{R}^{d}$, and $0<r \leq n$ be an integer. Let $\mathcal{P}(r)=$ $\left\{\left(P_{i}, \Delta_{i}\right) \mid 1 \leq i \leq r\right\}$ be a vertical simplicial $r$-partition of $P$. For each $p \in P$, let $\Delta(p)$ denote the ambient simplex $\Delta_{i}$ of $p$ in $\mathcal{P}(r)$. Then there is a set $\mathcal{L}(r)$ of at most $r^{(d-1)^{2}}$ vertical lines with the following property:

Let $0<\epsilon, \sigma \leq 1$ be so that $1 / \sigma \lll r \lll 1 / \epsilon^{d-1} \leq n, K \in \mathcal{K}(P, \epsilon)$ be an $\epsilon$-heavy convex set, $P_{K} \subseteq P \cap K$ be a subset of exactly $\lceil\epsilon n\rceil$ points, and $\Pi_{K} \subseteq\left(\begin{array}{c}P_{K} \\ d\end{array}\right)$ be a subset of $(d-1)$-simplices that satisfies $\left|\Pi_{K}\right| \geq \sigma\left(\begin{array}{c}\lceil\epsilon n\rceil \\ d\end{array}\right)$. Then at least one of the following claims hold:

1. There is a line $\ell \in \mathcal{L}(r)$ that pierces a subset $\Pi_{K}(\ell) \subseteq \Pi_{K}$ of cardinality $\Omega\left(\sigma^{\beta_{d-1}}\left(\begin{array}{c}\lceil\epsilon n\rceil \\ d\end{array}\right)\right)$, where the constant $\beta_{d-1} \geq 1$ is as defined in the Second Selection Theorem 3.2.

Furthermore, for each simplex $\left\{p_{1}, \ldots, p_{d}\right\} \in \Pi_{K}(\ell)$, the line $\ell$ is surrounded by the $d$ ambient simplices $\Delta\left(p_{1}\right), \ldots, \Delta\left(p_{d}\right)$ in $\mathcal{P}(r)$. See Figure 6 .

2. $K$ is $\epsilon_{\text {nar }}$-narrow with respect to $\mathcal{P}(r)$ for some $\epsilon_{\text {nar }} \geq C_{n a r} \sigma^{\beta_{d-1}} \epsilon$, where $C_{\text {nar }}>0$ is a constant that does not depend on $\epsilon, \sigma$, or $r$.

Notice that a convex set $K \in \mathcal{K}(P, \epsilon)$, with $P_{K}$ and $\Pi_{K}$ as in the hypothesis of Theorem 3.3 , can arise as part of a recursive instance $\mathcal{K}(P, \Pi, \epsilon, \sigma)$; in that case we have that $\Pi_{K}:=\Pi \cap\left(\begin{array}{c}P_{K} \\ d\end{array}\right)$, which clearly satisfies $\left|\Pi_{K}\right| \geq \sigma\left(\begin{array}{c}\lceil\epsilon n\rceil \\ d\end{array}\right)$. Nevertheless, the theorem does not rely on the existence of such a global set $\Pi \subseteq\left(\begin{array}{c}P \\ d\end{array}\right)$, and permits an "independent" choice of the subset $\Pi_{K} \subseteq\left(\begin{array}{c}P_{K} \\ d\end{array}\right)$ for each $\epsilon$-heavy set $K \in \mathcal{K}(P, \epsilon)$. This generality will be exploited in Section 5.5 .

In addition, our proof of Theorem 3.3 uses the following elementary properties.

Lemma 3.4. Let $\Sigma=\left\{\Delta_{1}, \ldots, \Delta_{k}\right\}$ be a collection of $k \geq 1$ d-dimensional simplices in $\mathbb{R}^{d}$, whose vertices are in general position 16 Furthermore, suppose that $\Sigma$ admits a transversal by a hyperplane. Then there is such a hyperplane that passes through some d distinct vertices of the simplices of $\Sigma$, and intersects the closure of every simplex in $\Sigma$. See Figure 7 (left).

\footnotetext{
${ }^{16}$ In particular, no $d+1$ of the vertices of $\Delta_{i}$, for $1 \leq i \leq k$, lie in the same hyperplane.
} 

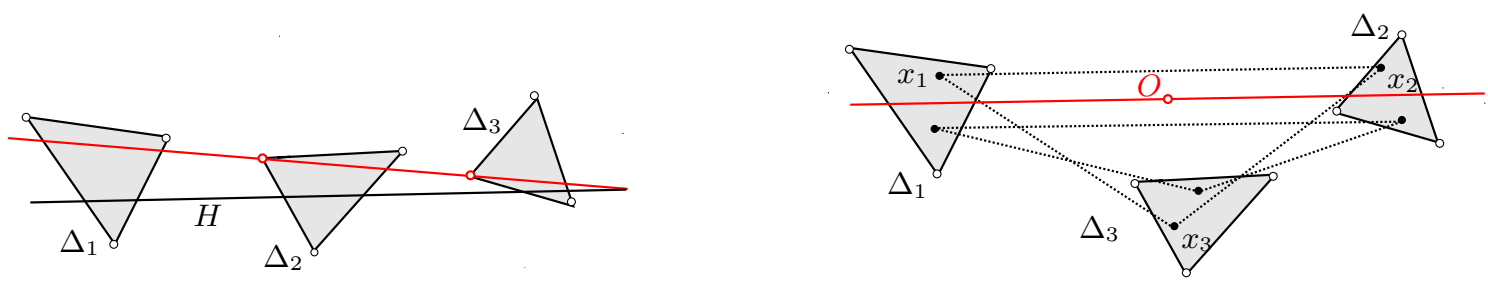

Figure 7: Left - Lemma 3.4 in dimension $d=2$ : moving a transversal hyperplane $H$ to $\Sigma=\left\{\Delta_{1}, \Delta_{2}, \Delta_{3}\right\}$ to a position in which it contains a pair of vertices while still intersecting the closure of each $\Delta_{i}$. Right: Proposition 3.6 for $d=3$ : The origin $O$ lies in the triangle $\triangle x_{1} x_{2} x_{3}$, for $x_{1} \in \Delta_{1}, x_{2} \in \Delta_{3}, x_{3} \in \Delta_{3}$, yet it is not surrounded by $\Delta_{1}, \Delta_{2}, \Delta_{3}$. The simplices $\Delta_{1}$ and $\Delta_{2}$ are crossed by a line through $O$.

Proof of Lemma 3.4. Let $H$ be any hyperplane transversal to $\Sigma$. We continuously translate $H$ in a fixed direction until the first time $H$ meets a vertex $v_{1}$ of a simplex $\Delta_{i_{1}} \in \Sigma$. We continuously rotate $H$ in a fixed direction around $v_{1}$, so the normal to $H$ is moving along a fixed great circle of $\mathbb{S}^{d-1}$, until the first time $H$ hits an additional vertex $v_{2}$ of a simplex $\Delta_{i_{2}} \in \Sigma$ (where $i_{1}$ is not necessarily different from $i_{2}$ ). We repeat this procedure $d-1$ times, each time adding a vertex $v_{j}$ of some simplex $\Delta_{i_{j}} \in \Sigma$, for $2 \leq j \leq d$, to the hyperplane $H$ which we rotate around the affine hull of the previously added vertices $v_{1}, \ldots, v_{j-1}$. (This is possible because the simplices of $\Sigma$ encompass a total of $k(d+1) \geq d+1$ vertices, whilst $H$ never contains more than $d$ of these vertices.) Once $H$ encounters a new vertex $v_{i_{j}}$, this vertex never leaves $H$, which reduces by 1 the available degrees of freedom that are available to the following rotation step. The process terminates when $H$ contains $d$ vertices, so its rotation is no longer possible.

Notice that, at every step, $H$ meets the closure of every simplex $\Delta_{j} \in \Sigma$, for the contact between $H$ and $\Delta_{j}$ cannot be lost before $H$ "gains" at least one of the $d+1$ vertices of $\Delta_{j}$.

Corollary 3.5. Let $\Sigma=\left\{\Delta_{1}, \ldots, \Delta_{d-1}\right\}$ be a collection of $d-1(d-1)$-dimensional simplices in $\mathbb{R}^{d-1}$ whose vertices are in general position with respect to themselves, and the origin $O \in \mathbb{R}^{d-1} \cdot 17$ Furthermore, suppose that $\Sigma$ admits a transversal by a $(d-2)$-plane that contains the origin $O$. Then there is such $a(d-2)$-plane that passes through $O$ and some $d-2$ vertices of the simplices of $\Sigma$, and intersects the closure of every simplex in $\Sigma$.

Proposition 3.6. Let $\Sigma=\left\{\Delta_{1}, \ldots, \Delta_{d}\right\}$ be a collection of $d(d-1)$-dimensional simplices in $\mathbb{R}^{d-1}$ whose vertices are in general position. Suppose that there exist points $x_{1} \in \Delta_{1}, \ldots, x_{d} \in \Delta_{d}$ so that conv $\left\{x_{1}, \ldots, x_{d}\right\}$ contains the origin $O \in \mathbb{R}^{d-1}$, but $O$ is not surrounded by $\Delta_{i}$, for $1 \leq i \leq d$. Then there is a $(d-2)$-plane that contains $O$ and crosses at least $d-1$ simplices of $\Sigma$. See Figure 7 (right).

Proof of Proposition 3.6. Since $O$ is not surrounded in $\mathbb{R}^{d-1}$ by $\Delta_{1}, \ldots, \Delta_{d}$, there exist points $y_{1} \in \Delta_{1}, \ldots, y_{d} \in \Delta_{d}$ so that $y \notin \operatorname{conv}\left(y_{1}, \ldots, y_{d}\right)$. For each $t \in[0,1]$, and each $1 \leq i \leq d$, we define the point $x_{i}(t):=(1-t) x_{i}+t y_{i}$ which clearly lies within $\Delta_{i}$. Let $\tau(t)$ denote the $(d-1)$-dimensional simplex $\operatorname{conv}\left(x_{1}(t), \ldots, x_{d}(t)\right)$ within $\mathbb{R}^{d-1}$. Notice that $\tau(0)=\operatorname{conv}\left\{x_{i} \mid 1 \leq i \leq d\right\}$ contains $O$, whereas $\tau(1)=\operatorname{conv}\left\{y_{i} \mid 1 \leq i \leq d\right\}$ no longer does. Thus, there must be $t^{*} \in(0,1)$ so that $y$ lies on the boundary of $\tau\left(t^{*}\right)$. By the general position, $O$ lies in the the relative interior of a $(d-2)$ simplex $\mu\left(t^{*}\right)$ of the boundary complex of $\tau\left(t^{*}\right)$. We can assume with no loss of generality that this

\footnotetext{
${ }^{17}$ In particular, no $d$ vertices of $\Delta_{i}$, for $1 \leq i \leq d-1$, lie in the same $(d-2)$-plane within $\mathbb{R}^{d-1}$, and no $d-1$ of their vertices lie in the same $(d-2)$-plane with the origin $O$.
} 

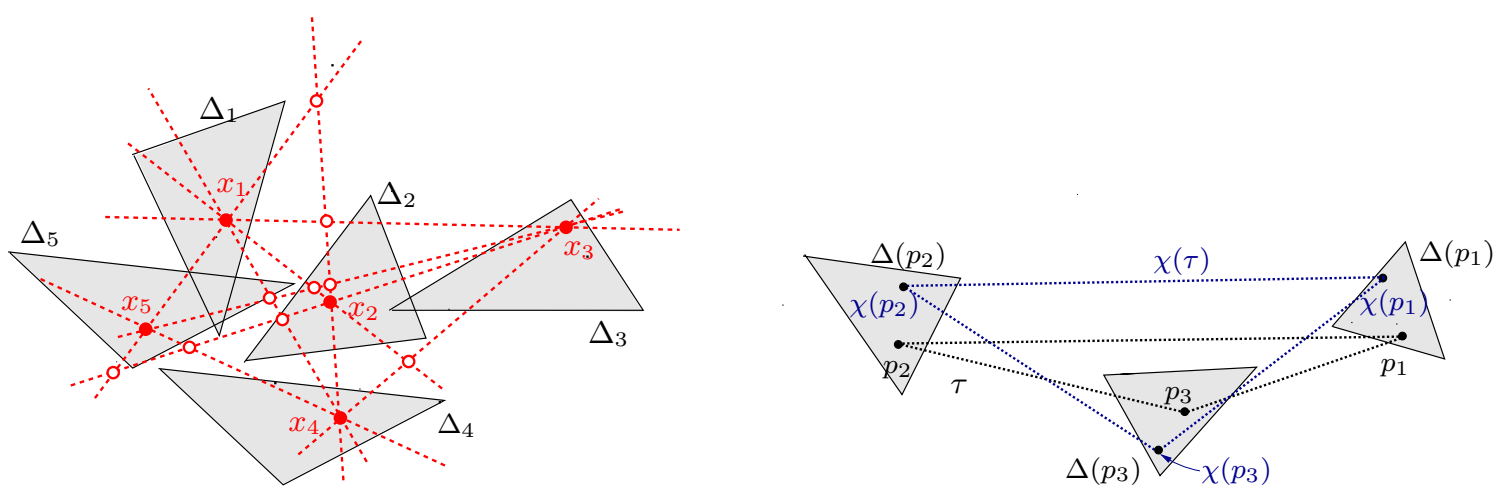

Figure 8: Proof of Theorem 3.3 in dimension $d=3$. Left: The set $X=\left\{x_{1}, \ldots, x_{r}\right\}$ with (part of) the induced set $Y$ in $\mathbb{R}^{2}$. Right: The triangle $\tau=\operatorname{conv}\left(p_{1}, p_{2}, p_{3}\right)$ in the vertical projection, and its representative $\chi(\tau)$ under the mapping $\chi: P_{K} \rightarrow \mathbb{R}^{2}$.

simplex is $\kappa^{*}=\operatorname{conv}\left(x_{1}\left(t^{*}\right), \ldots, x_{d-1}\left(t^{*}\right)\right)$, so its supporting $(d-2)$-plane aff $\left(\kappa^{*}\right)$ contains $y$ and also intersects $\Delta_{1}, \ldots, \Delta_{d-1}$ at the respective points $x_{1}\left(t^{*}\right), \ldots, x_{d-1}\left(t^{*}\right)$.

Proof of Theorem 3.3. For every $1 \leq i \leq r$ we pick a generic proxy point $x_{i} \in \Delta_{i}$, within $\mathbb{R}^{d-1}$. Denote $X:=\left\{x_{i} \mid 1 \leq i \leq r\right\}$. Let $Y$ be the set of the $(d-1)$-wise intersections of the $(d-2)$-planes that support (vertex disjoint) $(d-2)$-dimensional simplices in $\left(\begin{array}{c}X \\ d-1\end{array}\right)$; see Figure 8 (left). In other words,

$$
Y:=\left\{\bigcap_{i=1}^{d-1} \operatorname{aff}\left(B_{i}\right) \mid B_{1}, \ldots, B_{d-1} \in\left(\begin{array}{c}
X \\
d-1
\end{array}\right), \forall 1 \leq i \neq j \leq d-1: B_{i} \cap B_{j}=\emptyset\right\} .
$$

Let $\mathcal{L}(r)$ denote the set of all the vertical lines that result from lifting the points of $Y$. By definition, we have $|\mathcal{L}(r)| \leq r^{(d-1)^{2}}$, so it remains to show that $\mathcal{L}(r)$ satisfies the asserted properties.

Indeed, let $K$ be a convex set in $\mathcal{K}(P, \epsilon)$ that satisfies the hypothesis of the theorem. By the definition of $\mathcal{K}(P, \epsilon), K$ comes with a designated subset $P_{K} \subseteq P \cap K$ so that $\left|P_{K}\right|=\lceil\epsilon n\rceil$. Furthermore, $K$ is also endowed with a set $\Pi_{K} \subseteq\left(\begin{array}{c}P_{K} \\ d\end{array}\right)$ of cardinality $\left|\Pi_{K}\right| \geq \sigma\left(\begin{array}{c}P_{K} \\ d\end{array}\right)$.

We say that the set $K$ is degenerate if there exists $1 \leq i \leq r$ so that the part $P_{i}$ of $\mathcal{P}(r)$ encompasses more than $C_{d-1} \sigma^{\beta_{d-1}} n$ points of $P_{K}$; here the constants $C_{d-1}>0$ and $\beta_{d-1}>0$ are as determined in Theorem 3.2. Clearly, in this case $K$ is $\epsilon_{\text {nar-narrow in }} \mathcal{P}(r)$ provided that the constant $C_{n a r}>0$ in the second alternative of the theorem is at most $C_{d-1}$.

Assume then that $K$ is not degenerate. To invoke our Selection Theorem 3.2 for the $d$-uniform hypergraph $\left(P_{K}, \Pi_{K}\right)$, we define the embedding function $\chi: P_{K} \rightarrow X \subseteq \mathbb{R}^{d}$ so that $\chi(p)=x_{i}$ for each $p \in P_{i} \cap P_{K}$, and each $1 \leq i \leq r$. See Figure 8 (right). Clearly, $\chi$ is $\left(C_{d-1} \sigma^{\beta_{d-1}}\right)$ conforming. Hence, applying our Selection Theorem 3.2 for the hypergraph $\left(P_{K}, \Pi_{K}\right)$, and the embedding $\chi$, yields a point $y \in \mathbb{R}^{d-1}$ that pierces a subset $\Lambda(y)$ of $\Omega\left(\sigma^{\beta_{d-1}}\left(\begin{array}{c}\left|P_{K}\right| \\ d\end{array}\right)\right)$ simplices within $\left\{\operatorname{conv}(\chi(\tau)) \mid \tau \in \Pi_{K}\right\}$. Note that every element of $\Lambda(y)$ is a $(d-1)$-dimensional simplex with nonempty interior, of the form $\operatorname{conv}(\chi(\tau))$, for some $\tau=\operatorname{conv}\left(p_{1}, \ldots, p_{d}\right) \in \Pi_{K}$. (In particular $\chi(\tau)$ encompasses $d$ distinct points of $X$, so the $d$ ambient simplices $\Delta\left(p_{1}\right), \ldots, \Delta\left(p_{d}\right)$ are all distinct.)

Furthermore, since $1 / \sigma \lll 1 / \epsilon$ is bounded by an arbitrary small albeit fixed, degree of $1 / \epsilon$, and $1 / \epsilon^{d-1 / 2} \leq n$, we can assume that $\sigma \geq D_{d}(\lceil\epsilon n\rceil)^{-1 / t^{d}}$. As $\chi(P) \subseteq X$, there must exist $d-1$ pairwise disjoint $d$-sets $A_{i} \in\left(\begin{array}{c}X \\ d\end{array}\right)$ with subsets $B_{i} \in\left(\begin{array}{c}A_{i} \\ d-1\end{array}\right)$, so that our point $y$ is equal to $\bigcap_{i=1}^{d-1}$ aff $\left(B_{i}\right)$, and thereby belongs to $Y$. 

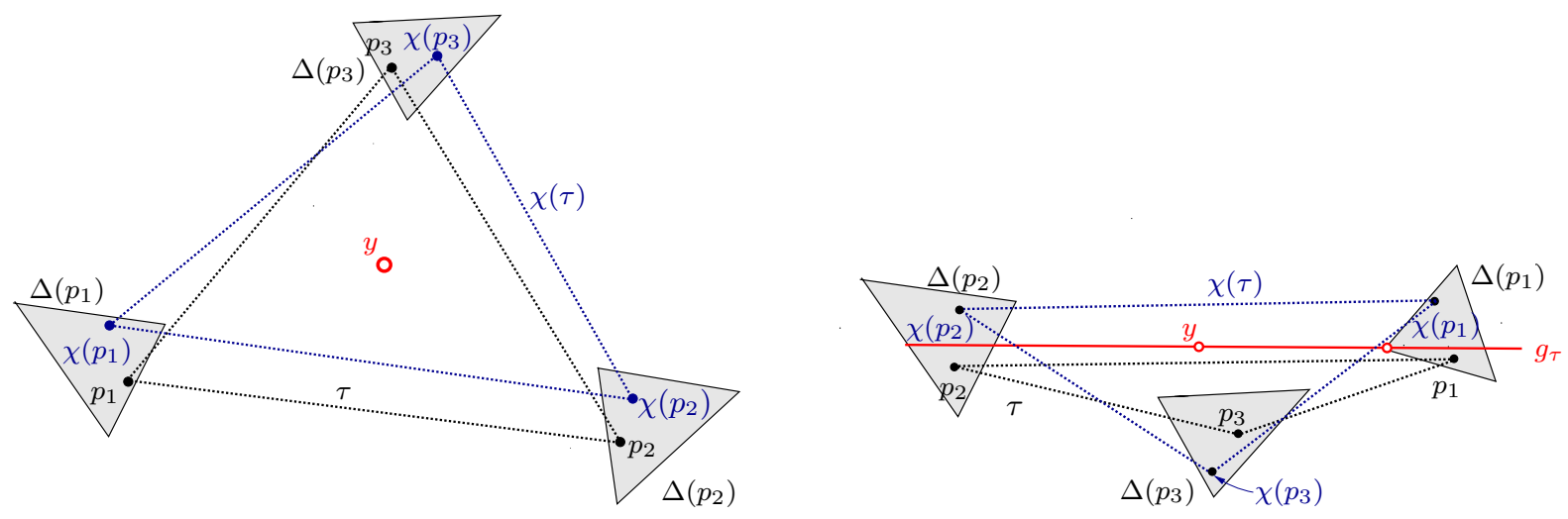

Figure 9: Proof of Theorem 3.3 (in dimension $d=3$ ). Left: Case $1-$ the $\operatorname{simplex} \tau=\operatorname{conv}\left(p_{1}, p_{2}, p_{3}\right)$ (shown in the vertical projection) belongs to $\Pi(y) \cap \Pi_{K}(\ell)$, so $y$ is surrounded by $\Delta\left(p_{1}\right), \Delta\left(p_{2}\right)$, and $\Delta\left(p_{3}\right)$; thus, $\tau$ is crossed by the vertical line $\ell$ over $y$. Right: Case $2-$ the $\operatorname{simplex} \tau=\operatorname{conv}\left(p_{1}, p_{2}, p_{3}\right)$ belongs to $\Pi(y) \backslash \Pi_{K}(\ell)$, so $y$ is not surrounded by $\Delta\left(p_{1}\right), \Delta\left(p_{2}\right)$, and $\Delta\left(p_{3}\right)$. The line $g_{\tau}$ passes through $y$ and a vertex of $\Delta\left(p_{1}\right)$, and crosses $\Delta\left(p_{2}\right)$.

Denote $\Pi(y):=\left\{\tau \in \Pi_{K} \mid \operatorname{conv}(\chi(\tau)) \in \Lambda(y)\right\}$. In other words, $\Pi(y) \subseteq \Pi_{K}$ is comprised of all the simplices $\tau \in \Pi_{K}$ whose proxies under $\chi$ belong to $\Lambda(y)$. Let $\ell=\ell(y)$ be the line in $\mathcal{L}(r)$ that is obtained by vertically lifting $y$, and let $\Pi_{K}(\ell) \subseteq \Pi(y)$ denote the subset of all the $(d-1)$-simplices $\tau=\left\{p_{1}, \ldots, p_{d}\right\}$ in $\Pi(y)$, so that $y$ is surrounded by their ambient simplices $\Delta\left(p_{1}\right), \ldots, \Delta\left(p_{d}\right) \subset \mathbb{R}^{d-1}$.

We distinguish between the following scenarios; see Figure 9.

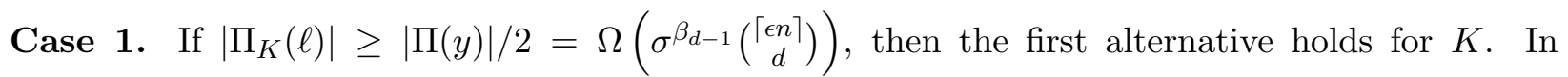
particular, every simplex $\tau=\operatorname{conv}\left(p_{1}, \ldots, p_{d}\right) \in \Pi_{K}(\ell)$ is crossed by the $\ell$ that we lifted from $y$, which is surrounded by $\Delta\left(p_{1}\right), \ldots, \Delta\left(p_{d}\right)$.

Case 2. If $\left|\Pi_{K}(\ell)\right|<|\Pi(y)| / 2$, we argue that $K$ falls into the second of the two proposed scenarios. To this end, we fix $\tau=\operatorname{conv}\left(p_{1}, \ldots, p_{d}\right) \in \Pi(y) \backslash \Pi_{K}(\ell)$. Since $y$ is not surrounded by the ambient simplices $\Delta\left(p_{1}\right), \ldots, \Delta\left(p_{d}\right)$ in $\mathbb{R}^{d-1}$ (yet lies in the simplex $\operatorname{conv}(\chi(\tau)$ ), whose vertices $\chi\left(p_{i}\right)$ lie in the respective ambient simplices $\left.\Delta\left(p_{i}\right)\right)$, Proposition 3.6 yields a $(d-2)$-plane through $y$ within $\mathbb{R}^{d-1}$, which intersects some $d-1$ of these simplices $\Delta\left(p_{i}\right)$, say $\Delta\left(p_{1}\right), \Delta\left(p_{2}\right), \ldots, \Delta\left(p_{d-1}\right)$. Furthermore, Corollary 3.5 yields such a $(d-2)$-plane $g_{\tau}$ that passes through some $d-2$ vertices of $\Delta\left(p_{1}\right), \Delta\left(p_{2}\right), \ldots, \Delta\left(p_{d-1}\right)$.

Notice that the supporting vertices of $g_{\tau}$ belong to at most $d-2$ simplices which, with no loss of generality, we assume to be amongst $\Delta\left(p_{1}\right), \ldots, \Delta\left(p_{d-2}\right)$. Also note that, for a fixed $y \in Y$, the $(d-2)$-plane $g_{\tau}$ can be guessed from $p_{1}, \ldots, p_{d-2}$ in at most $\left(\begin{array}{c}(d-2) d \\ d-2\end{array}\right)$ different ways (from among the vertices of the $d-2$ ambient simplices $\left.\Delta\left(p_{i}\right)\right)$. We therefore assign $\tau$ to this characteristic $(d-2)$-subset $S_{\tau}=\left\{p_{1}, \ldots, p_{d-2}\right\} \subset P_{K}$.

We repeat the above analysis for each simplex $\tau \in \Pi(y) \backslash \Pi_{K}(\ell)$. Since the overall number of distinct characteristic subsets $S_{\tau}$, over all $\tau \in \Pi(y) \backslash \Pi_{K}(\ell)$, is at most $\left(\begin{array}{c}\lceil\epsilon n\rceil \\ d-2\end{array}\right)$, by the pigeonhole principle there must be a $(d-2)$-subset $S \in\left(\begin{array}{c}P_{K} \\ d-2\end{array}\right)$ that is assigned $\Omega\left(\sigma^{\beta_{d-1}}\left(\begin{array}{c}\lceil\epsilon n\rceil \\ 2\end{array}\right)\right)$ of the $(d-1)$ simplices $\tau=\Pi(y) \backslash \Pi_{K}(\ell)$ (which then satisfy $S_{\tau}=S$ ). For each of these simplices $\tau$, one or both of the two vertices of $\left(\tau \backslash S_{\tau}\right)$ must belong to the partition sets $P_{i}$ whose ambient simplices $\Delta_{i}$ are crossed by $g_{\tau}$. Since a point of $P_{K}$ belongs to at most $\lceil\epsilon n\rceil$ distinct pairs $\tau \backslash S_{\tau}$, for $\tau \in \Pi(y) \backslash \Pi_{K}(\ell)$, 
the projections of at least $\Omega\left(\sigma^{\beta_{d-1}}\lceil\epsilon n\rceil\right)$ points in $P_{K}$ belong to the zone of $g_{\tau}$ in the projected simplicial $r$-partition $\mathcal{P}^{\perp}=\left\{\left(P_{1}^{\perp}, \Delta_{1}\right), \ldots,\left(P_{r}^{\perp}, \Delta_{r}\right)\right\}$. Therefore, with a suitably small choice of the constant $C_{n a r}>0$ in the definition $\epsilon_{\text {nar }}:=C_{n a r} \sigma^{\beta_{d-1}} \epsilon$, the set $K$ is again $\epsilon_{\text {nar }}$-narrow, and thereby falls into the second case of the theorem.

Combining Lemma 3.1 and Theorem 3.3 yields the following immediate bound on the transversal number of $\mathcal{K}(P, \Pi, \epsilon, \sigma)$.

Theorem 3.7. Let $r>0$ be an integer, $P$ be an $n$ point set in $\mathbb{R}^{d}, 0<\epsilon, \sigma \leq 1$, and $\Pi \subseteq\left(\begin{array}{l}P \\ d\end{array}\right)$. Then the set $\mathcal{K}(P, \Pi, \epsilon, \sigma)$ can be pierced by a net of size

$$
O\left(\frac{r^{(d-1)^{2}}|\Pi|}{\sigma^{\beta_{d-1}} \epsilon^{d} n^{d}}+r \cdot f\left(\sigma^{\beta_{d-1}} r^{1 /(d-1)} \cdot \epsilon\right)\right) .
$$

Notice that Theorem 3.7 readily yields a net of cardinality $o\left(1 / \epsilon^{d}\right)$ whenever $|\Pi|$ is substantially smaller than $\left(\begin{array}{l}n \\ d\end{array}\right)$, and $1 / \sigma \lll r$.

Proof of Theorem 3.7. We construct the vertical simplicial partition $\mathcal{P}=\left\{\left(P_{1}, \Delta_{1}\right), \ldots,\left(P_{r}, \Delta_{r}\right)\right\}$ as described in Section 3.1. We then invoke Lemma 3.1 to construct a net $N\left(\epsilon^{\prime}, r\right)$ of cardinality

$$
O\left(r \cdot f_{d}\left(\epsilon^{\prime} \cdot r^{1 /(d-1)}\right)\right)
$$

which pierces all the $\epsilon^{\prime}$-narrow sets $K \in \mathcal{K}(P, \Pi, \epsilon, \sigma)$ with respect to $\mathcal{P}(r)$; here $\epsilon^{\prime}=C \sigma^{\beta_{d-1}} \epsilon$ for some sufficiently small constant $C>0$, to be determined shortly, that does not depend on $\epsilon$ or $\sigma$.

We then invoke the Multiple Selection Theorem 3.3 for $\mathcal{P}$. Let $\mathcal{L}(r)$ be the resulting collection of at most $r^{(d-1)^{2}}$ vertical lines. For each $\ell \in \mathcal{L}(r)$ we construct a set $X_{\ell}:=\{\operatorname{conv}(\tau) \cap \ell \mid \tau \in \Pi\}$ of at most $|\Pi|$ intercept points of $\ell$ with the simplices of $\Pi$. We then construct a 1-dimensional strong $\lambda^{\prime}-$ net $N_{\ell}$ over the set $X_{\ell}$ with respect to intervals, for $\lambda^{\prime}=C^{\prime} \sigma^{\beta_{d-1}} \epsilon^{d} n^{d} /|\Pi|$; here $C^{\prime}>0$ is a suitably small constant that may depend on $d$ (but not on $\epsilon$ ). We then define $N^{\prime}:=\left(\bigcup_{\ell \in \mathcal{L}(r)} N_{\ell}\right) \cup N\left(\epsilon^{\prime}, r\right)$.

Since the cardinality of $N^{\prime}$ is obviously bounded by (5), it suffices to show that every convex set $K \in \mathcal{K}(P, \Pi, \epsilon, \sigma)$ is pierced by $N^{\prime}$. To this end, we select $C=C_{n a r}$, where $C_{n a r}>0$ is the constant in the second alternative of Theorem 3.3 .

Indeed, if $K$ falls into the first alternative of Theorem 3.3, there must be a line $\ell \in \mathcal{L}(r)$ that pierces $\Omega\left(\sigma^{\beta_{d-1}}\left(\begin{array}{c}\lceil\epsilon n\rceil \\ d\end{array}\right)\right)$ simplices of $\Pi_{K}:=\Pi \cap\left(\begin{array}{c}P_{K} \\ d\end{array}\right)$, each time at a point of $X_{\ell}$ within the segment $K \cap \ell$. Therefore, a suitable choice of the constant $C^{\prime}>0$ guarantees that $K$ is pierced by the respective net $\lambda^{\prime}$-net $N_{\ell} \subset N^{\prime}$.

On the other hand, if $K$ is $\epsilon^{\prime}$-narrow with respect to $\mathcal{P}(r)$, then $K$ is pierced by the net $N\left(\epsilon^{\prime}, r\right)$.

\section{Piercing the vertically $\delta$-convex sets}

Recall that every problem instance is of the form $\mathcal{K}=\mathcal{K}(P, \Pi, \epsilon, \sigma)$, and it consists of all the $\epsilon$-heavy convex sets with respect to $P$ that are $(\epsilon, \sigma)$-restricted to the underlying collection $\Pi$ of $m \geq \sigma\left(\begin{array}{l}n \\ d\end{array}\right)$ $(d-1)$-dimensional simplices. Throughout this section we keep the quantities $P, \Pi, \epsilon$, and $\sigma$, fixed.

In this section, we construct a small-size net $N_{\text {vcon }}$ in $\mathbb{R}^{d}$ that pierces the sub-family $\mathcal{K}_{\text {vcon }} \subset$ $\mathcal{K}(P, \Pi, \epsilon, \sigma)$ defined in the end of Section 2.4. Recall that we have $|\Pi| /\left(\begin{array}{l}n \\ d\end{array}\right) \leq \rho$, and the subfamily $\mathcal{K}_{\text {vcon }}$ encompasses all the sets $K \in \mathcal{K}(P, \Pi, \epsilon, \sigma)$ that are vertically $\delta$-convex, for $\delta=$ 
$C_{\mathrm{vcon}} / r^{d(d+1)+1}$; here the integer $r \lll 1 / \epsilon$ is proportional to an arbitrary small, albeit fixed, degree of $1 / \epsilon$.

Our goal is to obtain a recurrence for the maximum number of points that would be necessary to pierce every vertically $\delta$-convex set in $\mathcal{K}_{\mathrm{vcon}}$. Our bound will be expressed in the recursive terms of the form $f\left(\epsilon^{\prime}\right)$ and $f\left(\epsilon^{\prime}, \rho^{\prime}, \sigma^{\prime}\right)$ for $\epsilon^{\prime}=\Omega(\epsilon), \rho^{\prime} \leq \rho$, and $\sigma^{\prime}=\Theta(\sigma)$.

In the rest of this section, we denote $m:=|\Pi|$, and let $\mathcal{H}$ denote the set $\mathcal{H}(\Pi)=\{H(\tau) \mid \tau \in \Pi\}$ of all the hyperplanes (also $m$ in number) that are supported by the simplices of $\Pi$.

Our net $N_{\mathrm{vcon}}$ for the family $\mathcal{K}_{\mathrm{vcon}}$ is defined in incremental fashion, and its construction proceeds through several basic steps. Initially, we set $N_{\mathrm{vcon}}:=\emptyset$. At each subsequent step we add one or several, relatively elementary nets to $N_{\mathrm{vcon}}$, and remove from $\mathcal{K}_{\mathrm{vcon}}$ every convex set $K$ that is already pierced by $N_{\mathrm{vcon}}$. The construction terminates when no set in $\mathcal{K}_{\mathrm{vcon}}$ is left unpierced by $N_{\text {vcon }}$.

\section{$4.1 \quad$ A cutting for $\mathcal{A}(\mathcal{H})$}

Let $\mathcal{R}$ be a random $r$-sample of $\mathcal{H}$. Though the hyperplanes of $\mathcal{R}$ are not necessarily in general position (as those of $\mathcal{H}$ are certainly not), any single one of them contains at most $d$ points of $P$. Thus, they encompass a total of at most $r d$ points of $P$.

As reviewed in Section 5.3, this set $\mathcal{R}$ determines the arrangement $\mathcal{A}(\mathcal{R})$ of $\mathcal{R}$. The $d$ dimensional faces of $\mathcal{A}(\mathcal{R})$ constitute the decomposition of $\mathbb{R}^{d} \backslash(\bigcup R)$ into $O\left(r^{d}\right)$ open connected cells of overall complexity $O\left(r^{d}\right)$ [42, Section 6]. Let $P^{\prime} \subseteq P$ denote the subset of all the points that lie in the interiors of the cells of $\mathcal{A}(\mathcal{R})$. Since $n \geq 1 / \epsilon^{d-1 / 2} \ggg r$ (see Section 2.4), for each $K \in \mathcal{K}_{\mathrm{vcon}}$ the vast majority of the points of $P_{K}$ belong to $P^{\prime}$.

Applying the bottom-vertex triangulation of Section 2.3 to the cells of $\mathcal{A}(\mathcal{R})$ yields a simplicial decomposition $\mathcal{D}(\mathcal{R})$, that is, a collection of $O\left(r^{d}\right) d$-dimensional pairwise disjoint open simplices. The general position of $P$ implies that every point of $P^{\prime}$ lies in the interior of a unique simplex of $\mathcal{D}(\mathcal{R})$.

Definition. We fix a parameter $z$ that satisfies $1 \leq z \leq(2|\mathcal{D}(\mathcal{R})|)$. In Section 4.5, the value of $z$ will be set to $\Theta\left(r^{d-1 / 2}\right)$ so as to optimize the eventual recurrence for the vertically $\delta$-convex sets. Let us denote $P_{\varphi}:=P \cap \varphi$ for each $\varphi \in \mathcal{D}(\mathcal{R})$. We say that a simplex $\varphi \in \mathcal{D}(\mathcal{R})$ is heavy if $\left|P_{\varphi}\right| \geq\lceil n / z\rceil$. By definition, there exist at most $z$ heavy simplices in $\mathcal{D}(\mathcal{R})$.

We further subdivide every heavy simplex $\varphi$ of $\mathcal{D}(\mathcal{R})$ into $\left\lceil\left|P_{\varphi}\right| \frac{z}{n}\right\rceil$ open polyhedral cells $\varphi^{\prime}$ so that $\left|\varphi^{\prime} \cap P\right| \leq\lfloor n / z\rfloor$. To this end, we introduce $\left\lfloor\left|P_{\varphi}\right| \frac{z}{n}\right\rfloor$ parallel vertical walls. Let $\mathcal{D}_{z}(\mathcal{R})$ denote this more refined decomposition of $\mathbb{R}^{d}$. Furthermore, for every heavy simplex of $\mathcal{D}(\mathcal{R})$ we refer to all its off-shoots in $\mathcal{D}_{z}(\mathcal{R})$ as heavy cells. If a cell of $\mathcal{D}_{z}(\mathcal{R})$ is not heavy, we say that it is light. ${ }^{18}$ Notice that the refined decomposition $\mathcal{D}_{z}(\mathcal{R})$ still encompasses $O\left(r^{d}\right)$ cells, including at most $2 z$ heavy cells.

Note that every cell in $\mathcal{D}_{z}(\mathcal{R})$ is a convex polytope with at most $d+2$ facets, and at most $\left(\begin{array}{c}d+1 \\ 2\end{array}\right)+2\left(\begin{array}{c}d \\ 2\end{array}\right)=O\left(d^{2}\right)$ edges. Therefore, by the Strong Epsilon-Net Theorem 2.3. (the interior of) every cell $\varphi \in \mathcal{D}_{z}(\mathcal{R})$ is crossed by at most $C_{0}\left(\frac{m}{r} \log r\right)$ hyperplanes of $\mathcal{H}$, where $C_{0}>0$ is a constant that may depend on $d$.

Let $\mathcal{D}_{\text {heavy }}$ (resp., $\mathcal{D}_{\text {light }}$ ) denote the subset of heavy (resp., light) cells in $\mathcal{D}_{z}(\mathcal{R})$. Clearly, we have a total of at most

$$
I=C_{0}\left(\frac{m z}{r} \log r\right)
$$

\footnotetext{
${ }^{18}$ Note that, in our notation, the only light cells in $\mathcal{D}_{z}(\mathcal{R})$ are the simplices that have been inherited en toto from the bottom-vertex triangulation $\mathcal{D}(\mathcal{R})$.
} 
incidences between the elements of $\mathcal{D}_{\text {heavy }}$ and the hyperplanes of $\mathcal{H}$ that intersect them. Thus, an average hyperplane of $\mathcal{H}$ crosses at most

$$
\frac{I}{m}=O\left(\frac{z}{r} \log r\right)
$$

cells of $\mathcal{D}_{\text {heavy }}$ For any $h>0$ let $\mathcal{H}_{\geq h}$ be the subset of all the hyperplanes in $\mathcal{H}$ that cross at least $h I / m$ elements $\mathcal{D}_{\text {heavy }}$. Then Markov's Inequality readily yields the following estimate.

Proposition 4.1. Let $h>0$, then we have $\left|\mathcal{H}_{\geq h}\right|=O(m / h)$.

Let $\Pi_{\geq h} \subset \Pi$ denote the subset of all the simplices in $\Pi$ that support the hyperplanes of $\mathcal{H}_{\geq h}$.

In what follows, we set $h$ to be an arbitrary small, albeit fixed, degree of $1 / \epsilon$, that satisfies $h \lll r \lll 1 / \epsilon$, and construct an auxiliary net $N_{\geq h}$ of cardinality $f(\epsilon, \rho / h, \sigma / 2)$ that pierces all the convex sets in the family

$$
\mathcal{K}_{\geq h}=\mathcal{K}\left(P, \Pi_{\geq h}, \sigma / 2\right),
$$

which encompasses all the convex sets $K \in \mathcal{K}(P, \epsilon)$ that are $(\epsilon, \sigma / 2)$-restricted to the hypergraph $\left(P, \Pi_{\geq h}\right)$.

We include the points of $N_{\geq h}$ in $N_{\text {vcon }}$, and remove all the sets of $\mathcal{K}_{\geq h}$ from $\mathcal{K}_{\text {vcon }}$. In what follows, we focus on the remaining family, which we continue to denote by $\mathcal{K}_{\mathrm{vcon}}$. For each of the

remaining sets $K \in \mathcal{K}_{\mathrm{vcon}}$ we have that $\left|\left(\begin{array}{c}P_{K} \\ d\end{array}\right) \cap \Pi_{\geq h}\right|<(\sigma / 2)\left(\begin{array}{c}\lceil\epsilon n\rceil \\ d\end{array}\right)$. Hence, at least $(\sigma / 2)\left(\begin{array}{c}\lceil\epsilon n\rceil \\ d\end{array}\right)$ of the simplices of $\Pi_{K}$ belong to the complementary set

$$
\Pi^{\prime}:=\Pi \backslash \Pi_{\geq h},
$$

with the property that every hyperplane $\mathcal{H}\left(\Pi^{\prime}\right)$ crosses fewer than $h I / m$ simplices of $\mathcal{D}_{\text {heavy }}$. In other words, every remaining set in $\mathcal{K}_{\text {vcon }}$ is $(\epsilon, \sigma / 2)$-restricted to the hypergraph $\left(P, \Pi^{\prime}\right)$.

\subsection{Tracing the zones of convex sets via the hyperplanes of $\mathcal{H}\left(\Pi_{K}\right)$}

In this section we show that, with a possible exception of a certain sub-family of easily piercable convex sets, every remaining convex set $K \in \mathcal{K}$ can be assigned a hyperplane $H \in \mathcal{H}\left(P_{K}\right)$ whose zone in $\mathcal{D}_{z}(\mathcal{R})$ encompasses a large fraction of $P_{K}$.

The vertical partition $\mathcal{P}(s)$. As a preparation, we fix a natural parameter $s$ that satisfies $1 \lll r \lll s \lll 1 / \epsilon$, to be determined in the sequel, and construct an auxiliary vertical simplicial $s$-partition $\mathcal{P}(s)$ of the set $P$. (The value of $s$ will be proportional to an arbitrary small, albeit fixed, degree of $1 / \epsilon$.)

Pruning the narrow sets with respect to $\mathcal{P}(s)$. Denote

$$
\epsilon^{\prime}:=\frac{C^{\prime} \sigma^{\beta_{d-1}} \epsilon}{r}
$$

where $C^{\prime}>0$ is a sufficiently small constant to be determined in the sequel. In particular, we can assume that $C^{\prime}<C_{n a r} / 2^{\beta_{d-1}}$ (where $C_{n a r}$ is the constant in the second alternative of Theorem 3.3 .

Lemma 3.1 yields a net $N\left(\epsilon^{\prime}, s\right)$ of cardinality

$$
\left|N\left(\epsilon^{\prime}, s\right)\right|=O\left(s \cdot f\left(\epsilon^{\prime} \cdot s^{1 /(d-1)}\right)\right)=O\left(s \cdot f\left(\epsilon \cdot \frac{s^{1 /(d-1)} \sigma^{\beta_{d-1}}}{r}\right)\right)
$$




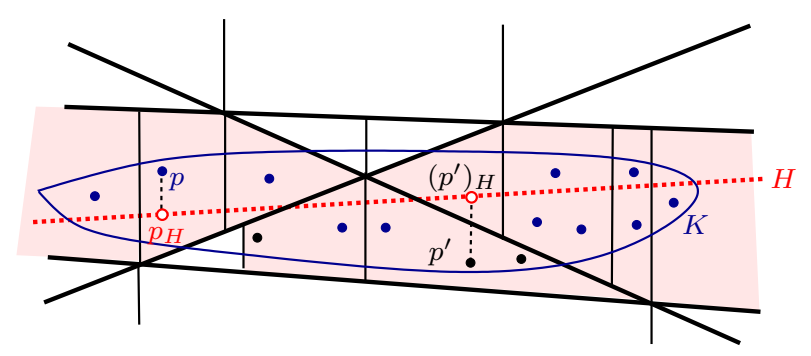

Figure 10: Left: The witness hyperplane $H=H_{K}$, its zone in the decomposition $\mathcal{D}_{z}(\mathcal{R})$ (red), and the witness set $A=A_{K}$ (blue). The point $p \in P_{K}$ can be added to $A$, as its $H$-projection $p_{H}$ belongs to the same cell of $\mathcal{A}(\mathcal{R})$. In contrast, the point $p^{\prime} \in P_{K} \backslash A$ is separated from its $H$-projection $\left(p^{\prime}\right)_{H}$ by a hyperplane of $\mathcal{R}$.

which pierces every convex set that is $\epsilon^{\prime}$-narrow with respect to $\mathcal{P}(s)$. We add $N\left(\epsilon^{\prime}, s\right)$ to $N_{\text {vcon }}$, and remove from $\mathcal{K}_{\mathrm{vcon}}$ every convex set that is pierced by $N\left(\epsilon^{\prime}, s\right)$. As a result, every set in the remaining family $\mathcal{K}_{\mathrm{vcon}}$ is $\epsilon^{\prime}$-spread with respect to $\mathcal{P}(s)$.

Definition. Let $x \in \mathbb{R}^{d}$ be a point, and $H$ be a non-vertical hyperplane in $\mathbb{R}^{d}$. Then $p_{H}$ denotes the vertical projection of $p$ onto $H$.

Let $C_{t r}>0$ be a fixed constant, to be determined in the sequel (namely, in Theorem 4.3). We say that a convex set $K \in \mathcal{K}_{\mathrm{vcon}}$ is traced in $\mathcal{D}_{z}(\mathcal{R})$ if there exists a hyperplane $H \in \mathcal{H}\left(\Pi^{\prime}\right)$, and a subset $A \subseteq P_{K}$ of cardinality

$$
|A| \geq C_{t r} \sigma^{\beta_{d-1}} \epsilon n
$$

so that the following conditions are satisfied:

1. $A$ is contained in the zone of $H$ in $\mathcal{D}_{z}(\mathcal{R})$.

2. For each point $p \in A$, its projection $p_{H}$ lies in the same cell of the arrangement $\mathcal{A}(\mathcal{R})$ as $p$; see Figure 10

To every set $K \in \mathcal{K}$ that is traced in $\mathcal{D}_{z}(\mathcal{R})$, we assign a single witness hyperplane $H_{K} \in \mathcal{H}\left(\Pi^{\prime}\right)$ and a single witness subset $A_{K} \subset P_{K}$ which satisfy the above two properties.

Pruning the untraced sets. Since every remaining set $K \in \mathcal{K}_{\mathrm{vcon}}$ is $\epsilon^{\prime}$-spread in $\mathcal{P}(s)$ (and $\epsilon^{\prime}$ is much smaller than $\left.C_{n a r}(\sigma / 2)^{\beta_{d-1}} \epsilon\right)$, our Multiple Selection Theorem 3.3 yields a set $\mathcal{L}(s)$ of at most $s^{(d-1)^{2}}$ vertical lines with the following property: For every convex set in $K \in \mathcal{K}_{\text {vcon }}$, there is a line $\ell \in \mathcal{L}(s)$ that pierces $\Omega\left(\sigma^{\beta_{d-1}} \epsilon^{d} n^{d}\right)$ of the simplices in the set $\Pi_{K}^{\prime}:=\Pi^{\prime} \cap\left(\begin{array}{c}P_{K} \\ d\end{array}\right)$ (whose cardinality is at least $\left.\frac{\sigma}{2}\left(\begin{array}{c}\left|P_{K}\right| \\ d\end{array}\right)\right)$.

For each vertical line $\ell \in \mathcal{L}(s)$ let us denote $X_{\ell}:=\{\ell \cap H \mid H \in \mathcal{R}\}$, and set

$$
X:=\bigcup_{\ell \in \mathcal{L}(s)} X_{\ell}
$$

We add to $X$ every vertex of $\mathcal{D}_{z}(\mathcal{R})$. Altogether, this yields a set of cardinality

$$
|X| \leq s^{(d-1)^{2}} r+O\left(r^{d}\right)=O\left(s^{(d-1)^{2}} r\right) .
$$


We then define a new set of simplices

$$
\Pi_{X}:=\left(\begin{array}{c}
P \\
d-1
\end{array}\right) * X=\left\{\operatorname{conv}(\kappa \cup x) \mid \kappa \in\left(\begin{array}{c}
P \\
d-1
\end{array}\right), x \in X\right\}
$$

with the induced set $\mathcal{H}_{X}:=\mathcal{H}\left(\Pi_{X}\right)$ of supporting hyperplanes. Notice that

$$
\left|\mathcal{H}_{X}\right|=\left|\Pi_{X}\right|=O\left(n^{d-1}|X|\right)=O\left(n^{d-1} s^{(d-1)^{2}} r\right) .
$$

For every $\ell \in \mathcal{L}(s)$ we construct the point set

$$
Y_{\ell}=:\left\{\ell \cap H \mid H \in \mathcal{H}_{X}\right\}
$$

which encompasses all the $\ell$-intercepts of the hyperplanes of $\mathcal{H}_{X}$, so $\left|Y_{\ell}\right|=O\left(n^{d-1} s^{(d-1)^{2}} r\right)$.

Finally, for every $\ell \in \mathcal{L}(s)$ we construct a net $N_{\ell}$ which encompasses every $\left\lceil C_{t r}^{\prime} \sigma^{\beta_{d-1}} \epsilon^{d-1} n^{d-1}\right\rceil$ th point of $Y_{\ell}$, where $C_{t r}^{\prime}>0$ is a constant to be fine-tuned in Proposition 4.3 , and set

$$
N_{t r}:=\bigcup_{\ell \in \mathcal{L}(s)} N_{\ell}
$$

This immediately yields the following bound of the cardinality of $N_{t r}$.

Proposition 4.2. The above net $N_{\text {tr }}$ has cardinality

$$
O\left(\frac{s^{2(d-1)^{2}} r}{\sigma^{\beta_{d-1}} \epsilon^{d-1}}\right)
$$

Choosing $C^{\prime}, C_{t r}$, and $C_{t r}^{\prime}$. We are at last ready to select the constants $C^{\prime}>0$ in $(6), C_{t r}>0$ in (8), and $C_{t r}^{\prime}>0$ in the definition of the net $N_{t r}$. Their choice is prescribed by the following proposition.

Theorem 4.3. There exists a choice of the constants $C^{\prime}>0, C_{t r}>0$, and $C_{t r}^{\prime}>0$, so that the following property holds: The net $N_{\text {tr }}$ pierces every $\epsilon^{\prime}$-spread convex set in $\mathcal{K}_{\mathrm{vcon}}$ that is not traced in $\mathcal{D}_{z}(\mathcal{R})$.

Proof. Let $K \in \mathcal{K}_{\text {vcon }}$ be any $\epsilon^{\prime}$-spread convex set that is not traced in $\mathcal{D}_{z}(\mathcal{R})$. We show that $K$ is pierced by the net $N_{t r}$ given a suitable choice of $C^{\prime}, C_{t r}$ and $C_{t r}^{\prime}$ (which will not depend on the choice of $K)$.

Indeed, since $K$ is $\epsilon^{\prime}$-spread (and $\epsilon^{\prime}$ in $(6)$ is much smaller than $C_{n a r}(\sigma / 2)^{\beta_{d-1}} \epsilon$ ), our Multiple Selection Theorem 3.3 yields a line $\ell_{0} \in \mathcal{L}(s)$ that pierces a subset $\Pi_{K}^{\prime}\left(\ell_{0}\right)$ of $\Omega\left(\sigma^{\beta_{d-1}}\left(\begin{array}{c}\lceil\epsilon n\rceil \\ d\end{array}\right)\right)$ simplices of $\Pi_{K}^{\prime}, 19$

Definition. Let $\kappa$ be a $(d-2)$-simplex in $\left(\begin{array}{c}P_{K} \\ d-1\end{array}\right)$. We say that a simplex $\tau \in \Pi_{K}^{\prime}\left(\ell_{0}\right)$ is adjacent to $\kappa$ if $\kappa$ encompasses $d-1$ vertices of $\tau$.

We say that $p \in P_{K}$ is a neighbour of $\kappa$ if the $(d-1)$-dimensional simplex $\operatorname{conv}(\kappa \cup\{p\})$ belongs to $\Pi_{K}^{\prime}\left(\ell_{0}\right)$.

Let $\Gamma(\kappa) \subseteq P_{K}$ denote the subset of all the neighbours of $\kappa \in\left(\begin{array}{c}P_{K} \\ d-1\end{array}\right)$. (Notice that the points of $\Gamma(\kappa)$ correspond to the simplices of $\Pi_{K}^{\prime}\left(\ell_{0}\right)$ that are adjacent to $\kappa$.)

\footnotetext{
${ }^{19}$ As the hyperplanes of $\mathcal{R}$ encompass a total of at most $r d$ points of $P$, we can assume that all the vertices of the simplices of $\Pi_{K}^{\prime}\left(\ell_{0}\right)$ belong to the set $P^{\prime}=P \backslash(\bigcup \mathcal{R})$ and, therefore, lie in the interiors of the cells of $\mathcal{D}_{z}(\mathcal{R})$.
} 


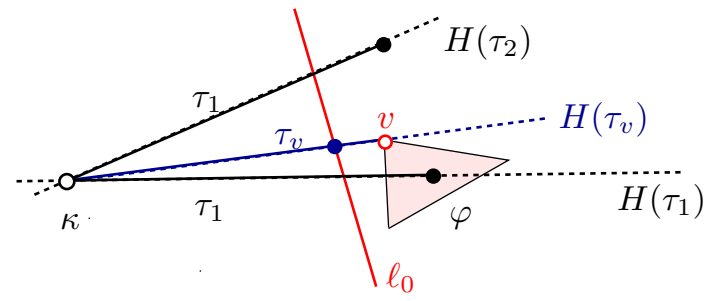

Figure 11: Proof of Theorem 4.3 - Case (i). The $(d-2)$-simplex $\kappa$ is not very good (view in a direction parallel to $\kappa)$. There exist $(d-1)$-simplices $\tau_{1}, \tau_{2} \in \Pi_{K}^{\prime}\left(\ell_{0}\right)$ so that $H\left(\tau_{1}\right)$ crosses a simplex $\varphi \in \mathcal{D}_{z}(\mathcal{R})$ that is missed by $H\left(\tau_{2}\right)$. There is a vertex $v$ of $\varphi$, and a simplex $\tau_{v}=\operatorname{conv}(\kappa \cup\{v\}) \in \Pi_{X}$, such that the hyperplane $H\left(\tau_{v}\right) \in \mathcal{H}_{X}$ meets $\ell_{0}$ at a point of $Y_{\ell_{0}}$ between $\tau_{1} \cap \ell_{0}$ and $\tau_{2} \cap \ell_{0}$.

We say that $\kappa \in\left(\begin{array}{c}P_{K} \\ d-1\end{array}\right)$ is $\operatorname{good}$ if $|\Gamma(\kappa)| \geq C_{\text {good }} \cdot \sigma^{\beta_{d-1}} \epsilon n$; here $C_{\text {good }}>0$ is yet another constant to be fine-tuned to suit this proof. By the pigeonhole principle, there exists a choice $C_{\text {good }}$ (independent of $K)$ so that at least $\Omega\left(\sigma^{\beta_{d-1}}\left(\begin{array}{l}\lceil\epsilon n\rceil \\ d-1\end{array}\right)\right)$ of the $(d-2)$-simplices in $\left(\begin{array}{c}P_{K} \\ d-1\end{array}\right)$ are good.

We say that a good $(d-2)$-simplex $\kappa$ is very good if for every two simplices $\tau_{1}, \tau_{2} \in \Pi_{K}^{\prime}\left(\ell_{0}\right)$ that are adjacent to $\kappa$, their supporting hyperplanes $H_{1}:=H\left(\tau_{1}\right)$ and $H_{2}:=H\left(\tau_{2}\right)$ have identical zones in $\mathcal{D}_{z}(\mathcal{R})$.

Case (i). Suppose that at least half of the good $(d-2)$-simplices $\kappa \in\left(\begin{array}{c}P_{K} \\ d-1\end{array}\right)$ are not very good, and fix any such, not very good, simplex $\kappa$. Then there is a pair of simplices $\tau_{1}, \tau_{2} \in \Pi_{K}^{\prime}\left(\ell_{0}\right)$ that are adjacent to $\kappa$, and whose supporting planes $H\left(\tau_{1}\right)$ and $H\left(\tau_{2}\right)$ have non-identical zones within $\mathcal{D}_{z}(\mathcal{R})$. Assume with no loss of generality that there is a cell $\varphi \in \mathcal{D}_{z}(\mathcal{R})$ that is intersected by $H\left(\tau_{1}\right)$ but not $H\left(\tau_{2}\right)$.

We continuously rotate a hyperplane $H$ around the $(d-2)$-flat aff $(\kappa)$, from $H\left(\tau_{1}\right)$ to $H\left(\tau_{2}\right)$, so that $H$ never ceases intersecting the vertical line $\ell_{0}$. (As is easy to check, there is exactly one direction that permits such a continuous rotation around $\kappa$.) By continuity, there must be a point in time when the intersection $H \cap \varphi$ is about to vanish from $H$. By the general position, this can only happen when $H$ coincides with a vertex $v$ of $\varphi$ and, therefore, supports the simplex $\tau_{v}:=\operatorname{conv}(\kappa \cup\{v\})$. Since $v$ has been included in $X, \tau_{v}$ belongs to $\Pi_{X}$. Hence, the set $Y_{\ell_{0}}$ must include the point $H \cap \ell_{0}$, which clearly lies in the vertical interval between the points $\tau_{1} \cap \ell_{0}$ and $\tau_{2} \cap \ell_{0}$, and well within $K \cap \ell_{0}$. See Figure 11 .

Repeating the above argument for every good $(d-2)$-simplex $\kappa \in\left(\begin{array}{c}P_{K} \\ d-1\end{array}\right)$ that is not very good, yields $\Omega\left(\sigma^{\beta_{d-1}} \epsilon^{d-1} n^{d-1}\right)$ vertices of $Y_{\ell_{0}}$ that lie within the interval $K \cap \ell_{0}$. However, in such a case, and provided the constant $C_{t r}^{\prime}>0$ is small enough, the interval $K \cap \ell_{0} \subseteq K$ must contain a point of the net $N_{\ell_{0}} \subset N_{t r}$.

Case (ii). Hence, we can assume in what follows, that more than half of the good $(d-2)$-simplices $\kappa$ in $\left(\begin{array}{c}P_{K} \\ d-1\end{array}\right)$ are very good. Notice that for each of these $\Omega\left(\sigma^{\beta_{d-1}} \epsilon^{d-1} n^{d-1}\right)$ very good $(d-2)$ simplices $\kappa \in\left(\begin{array}{c}P_{K} \\ d-1\end{array}\right)$, all of their $\Omega\left(\sigma^{\beta_{d-1}} \epsilon n\right)$ neighbours $p \in \Gamma(\kappa)$ must belong to the zone of every hyperplane $H(\tau)$ that supports an adjacent simplex $\tau \in \Pi_{K}^{\prime}\left(\ell_{0}\right)$ (so that $\kappa \subset \tau$ ). Summing over all the very good $(d-2)$-simplices $\kappa \in\left(\begin{array}{c}P_{K} \\ d-1\end{array}\right)$ yields a total of $\Omega\left(\sigma^{2 \beta_{d-1}} \epsilon^{d} n^{d}\right)$ candidate hyperplanes $H \in \mathcal{H}\left(\Pi_{K}^{\prime}\right)$ that, given a suitably small choice of $C_{t r}>0$, satisfy the first criterion of tracing $K$ (with $A=\Gamma(\kappa)$ ).

Definition. We say that a very good $(d-2)$-simplex $\kappa \in\left(\begin{array}{c}P_{K} \\ d-1\end{array}\right)$ is excellent if there is a hyperplane $H \in \mathcal{H}\left(\Pi_{K}^{\prime}\right)$ that supports an adjacent simplex of $\kappa$, and satisfies the following property: For at least half of the points $p \in \Gamma(\kappa)$, both $p$ and its projection $(p)_{H}$ lie in the same cell of $\mathcal{A}(\mathcal{R})$. 


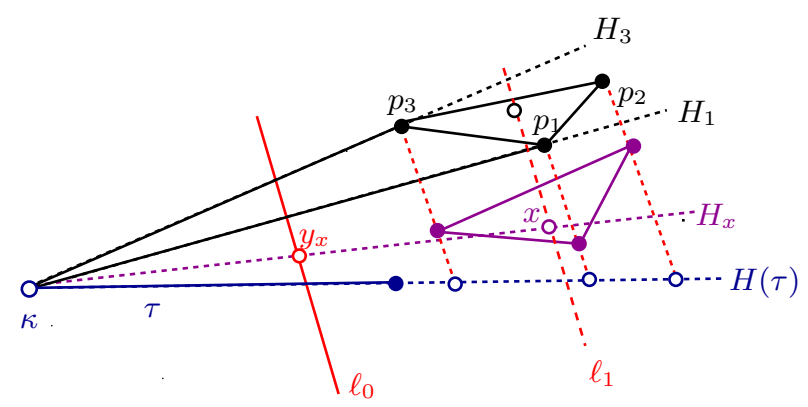

Figure 12: Proof of Claim 4.4 in dimension 3 - Case (ii) (view in the direction of $\kappa$ ). The simplex $\tau=\operatorname{conv}\left(p_{1}, p_{2}, p_{3}\right)$ in $\left(\begin{array}{c}P_{K}^{\prime \prime} \\ 3\end{array}\right)$ is crossed by the line $\ell_{1} \in \mathcal{L}(s)$. For each point $p_{i}$, its projections $\left(p_{i}\right)_{G}$ (purple) and $\left(p_{i}\right)_{H(\tau)}$ are depicted. The point $x=\ell_{1} \cap G$ lies in conv $\left(\left(p_{1}\right)_{G},\left(p_{2}\right)_{G},\left(p_{3}\right)_{G}\right)$, so the plane $H_{x}=\operatorname{aff}(\kappa \cup\{x\})$ meets $\ell_{0}$ between $H_{1}$ and $H_{3}$, and within $K \cap \ell_{0}$.

If there is at least one excellent $(d-2)$-simplex $\mu \in\left(\begin{array}{c}P_{K} \\ d-1\end{array}\right)$, then the set $K$ is duly traced given a sufficiently small choice of the constant $C_{t r}>0$. Therefore, we can assume in what follows, that no $(d-2)$-simplex in $\left(\begin{array}{c}P_{K} \\ d-1\end{array}\right)$ is excellent. We fix a very good simplex $\kappa \in\left(\begin{array}{c}P_{K} \\ d-1\end{array}\right)$, and consider all the $|\Gamma(\kappa)|$ hyperplanes $H(\tau)$ that support the adjacent $(d-1)$-simplices $\tau$ of $\kappa$ in $\Pi_{K}^{\prime}\left(\ell_{0}\right)$. Fix such a hyperplane $H=H(\tau)$ with the lowest possible intercept $H(\tau) \cap \ell_{0}$. Clearly, the point $H \cap \ell_{0}$ belongs to the interval $K \cap \ell_{0}$ (for $\ell_{0}$ crosses the supporting simplex $\tau$ of $H$ ).

Let $B_{K} \subset \Gamma(\kappa)$ be the subset of the neighbouring points $p$ of $\kappa$ so that the vertical segment $p p_{H}$ crosses one or more hyperplanes of $\mathcal{R}$. Notice that for every point $p \in \Gamma(\kappa) \backslash B_{K}$, both $p$ and $p_{H}$ lie in the same cell of $\mathcal{A}(\mathcal{R})$. Hence, we have that $\left|B_{K}\right| \geq|\Gamma(\kappa)| / 2$ (or, else, $\kappa$ would have been excellent, so $K$ would have been traced in $\mathcal{D}_{z}(\mathcal{R})$ ).

Assume with no loss of generality, that for at least $\left|B_{K}\right| / 2$ of the points $p \in B_{K}$ lie above $H$. We remove from $B_{K}$ all the points that lie below $H$, which leaves us with a set $B_{K}$ of cardinality $\left|B_{K}\right| \geq|\Gamma(\kappa) / 4|$. By the Pigeonhole Principle, there is a sample hyperplane $G \in \mathcal{R}$ that crosses at least $\left|B_{K}\right| / r$ of the segments $\left\{p p_{H} \mid p \in B_{K}\right\}$.

Let $P_{K}^{\prime \prime} \subseteq B_{K}$ denote the subset of all the points in $B_{K}$ whose respective segments $p p_{H}$ cross $G$. By definition, we have that $\left|P_{K}^{\prime \prime}\right| \geq \epsilon^{\prime \prime}$, for some $\epsilon^{\prime \prime}=\Omega\left(\sigma^{\beta_{d-1}} \epsilon n / r\right)$. Applying the Multiple Selection Theorem 3.3 again (this time for the partition $\mathcal{P}(s)$, the family $\mathcal{K}\left(P, \epsilon^{\prime \prime}\right)$, and with $\sigma=1$ ), assuming that $K$ is $\epsilon^{\prime}$-spread in $\mathcal{P}(s)$, and choosing a sufficiently small $C^{\prime}>0$ in (6), yields a line $\ell_{1} \in \mathcal{L}(s)$ that pierces $\Omega\left(\left(\begin{array}{c}\left\lceil\epsilon^{\prime \prime} n\right\rceil \\ d\end{array}\right)\right)$ simplices of $\left(\begin{array}{c}P_{K}^{\prime \prime} \\ d\end{array}\right)$.

Denote $x:=\ell_{1} \cap G$. Note that $x$ belongs to the previously defined set $X$, so the simplex $\tau_{x}:=\operatorname{conv}(\kappa \cup\{x\})$ belongs to $\Pi_{X}$. Let $H_{x}:=H\left(\tau_{x}\right)$ be the supporting plane of $\tau_{x}$, so the intersection point $y_{x}=H_{x} \cap \ell_{0}$ belongs to the set $Y_{\ell_{0}}$.

Claim 4.4. The above point $y_{x}=H_{x} \cap \ell_{0}$ must lie in the interval $K \cap \ell_{0}$.

Proof of Claim 4.4. Fix any $(d-1)$-simplex $\tau=\operatorname{conv}\left\{p_{1}, \ldots, p_{d}\right\} \in\left(\begin{array}{c}P_{K}^{\prime \prime} \\ d\end{array}\right)$ that is crossed by $\ell_{1}$. As $P_{K}^{\prime \prime} \subset \Gamma(\kappa)$, each $p_{i}$ determines a hyperplane $H_{i} \in \mathcal{H}\left(\Pi_{K}^{\prime}\right)$ which supports a simplex $\tau_{i}=$ $\operatorname{conv}\left(\kappa \cup\left\{p_{i}\right\}\right)$ of $\Pi_{K}^{\prime}(\ell) \subset \Pi_{K}^{\prime}$. Assume with no loss of generality that the points $p_{i}$ are numbered in the vertically increasing order of the respective intersections $H_{i} \cap \ell_{0}$, for $1 \leq i \leq d$, and note that each of these intersections lies above $H \cap \ell_{0}$ and within the interval $K \cap \ell_{0}$. See Figure 12 .

We continuously rotate the hyperplane $H$ around $\kappa$ so that the intersection point $H \cap \ell_{0}$ is moving towards $H_{d} \cap \ell_{0}$, within the interval $K \cap \ell_{0}$. Notice that in the beginning of this rotation, 
all the projections $\left(p_{i}\right)_{G}$, for $1 \leq i \leq d$, lie entirely above $H$ (for they lie in the vertical segments whose lowermost endpoints belong to $H$ ). However, the points $\left(p_{i}\right)_{G}$ lie below $H$ by the time $H$ reaches $p_{d}$ (and, thereby, coincides with $H_{d}$ ). Since $\ell_{1}$ pierces $\tau$, the intersection point $x=\ell_{1} \cap G$ lies in conv $\left(\left(p_{1}\right)_{G}, \ldots,\left(p_{d}\right)_{G}\right)$. Hence, the hyperplane $H$ must pass through $x$ during its continuous rotation. When that happens, $H$ coincides with $H_{x}$, which places the intersection point $y_{x}=H_{x} \cap \ell_{0}$ in the interval $K \cap \ell_{0}$ and completes the proof of this claim.

Back to the proof of Theorem 4.3. To recap, there exist $\Omega\left(\sigma^{\beta_{d-1}} \epsilon n\right.$ ) very good (yet not excellent) $(d-2)$-simplices $\kappa$, and each of them contributes, via the above claim, at least one point of $Y_{\ell_{0}}$ to the interval $K \cap \ell_{0}$. It is easy to see that no pair of very good $(d-2)$-simplices contribute the same point to $Y_{\ell_{0}}$ provided that the set $P$ is in general position. Thus, given a suitably small choice of the constant $C_{t r}^{\prime}>0$ in the definition of $N_{t r}$, the interval $K \cap \ell_{0}$ must contain at least one point of the net $N_{\ell_{0}} \subseteq N_{t r}$, which concludes the proof of Theorem 4.3.

We add $N_{t r}$ to $N_{\mathrm{vcon}}$ and remove from $\mathcal{K}_{\mathrm{vcon}}$ every set that is pierced by $N_{t r}$. As a result, every remaining set $K \in \mathcal{K}_{\text {conv }}$ is traced in $\mathcal{D}_{z}(\mathcal{R})$ (in addition to being $\epsilon^{\prime}$-spread); in particular, $P_{K}$ contains a large witness set $A_{K}$ that is entirely contained in the zone of the witness hyperplane $H_{K} \in \Pi_{K}^{\prime}$ within $\mathcal{D}_{z}(\mathcal{R})$. Furthermore, by our prior assumption, the zone of $H_{K}$ in $\mathcal{D}_{\text {heavy }}$ is comprised of at most $h I / m$ cells.

Definition. To further subdivide the remaining family $\mathcal{K}_{\text {vcon }}$, let us denote $\mathcal{F}:=\left\{K \in \mathcal{K}_{\text {vcon }} \mid\right.$ $\left.\left|A_{K} \cap\left(\bigcup \mathcal{D}_{\text {heavy }}\right)\right| \geq\left|A_{K}\right| / 2\right\}$, and $\mathcal{G}:=\mathcal{K}_{\text {vcon }} \backslash \mathcal{F}$. Namely, the former family $\mathcal{F}$ encompasses all the sets $K \in \mathcal{K}_{\mathrm{vcon}}$ so that at least half of the points in their witness sub-sets $A_{K}$ come from the cells of $\mathcal{D}_{\text {heavy }}$. Accordingly, the complementrary family $\mathcal{G}$ is comprised of the remaining convex sets $K \in \mathcal{K}_{\mathrm{vcon}}$, so that at least half of their points in $A_{K}$ come from $\bigcup \mathcal{D}_{\text {light }}$.

Piercing the families $\mathcal{F}$ and $\mathcal{G}$ - fast forward. In the following Sections 4.3 and 4.4 , we construct respective nets $N_{\mathcal{F}}$ and $N_{\mathcal{G}}$ for the above families $\mathcal{F}$ and $\mathcal{G}$. In either case, our analysis employs the basic divide-and-conquer strategy with respect to the underlying set $P$ which was previously used to establish Lemma 3.1. As a result, we bound the cardinality of our nets via a sum of the more basic terms $f\left(\epsilon^{\prime}\right)$, where $\epsilon^{\prime}$ is much larger than $\epsilon$.

Specifically, to pierce the sets $K \in \mathcal{F}$ we use that the points of the respective witness sets $A_{K}$ are localized in at most $h I / m=\tilde{O}(z / r)=o\left(r^{d}\right)$ cells of $\mathcal{D}_{\text {heavy }}$, which immediately leads to a favourable recurrence. However, the argument does not extend to the sets $K \in \mathcal{G}$, as their witness sets $A_{K}$ can be spread out over a potentially larger number of cells in $\mathcal{D}_{\text {light. }}$ Instead, we use the vertical $\delta$-convexity of such sets $K$ (along with the properties of $A_{K}$ and $H_{K}$ ) in order to find a $(d-1)$-dimensional convex set $U_{K} \subseteq H_{K}$ so that a large fraction of the points of $A_{K}$ lie in the zone of the relative boundary $\partial U_{K}$ of $U_{K}$ in $\mathcal{A}(\mathcal{R})$. Furthermore, Theorem 2.7 implies the zone $\partial U_{K}$ is comprised of $O\left(r^{d-2}\right)$ cells of $\mathcal{A}(\mathcal{R})$. Unfortunately, some of these cells may have large complexity and, therefore, give rise to multiple light cells in the bottom-vertex triangulation $\mathcal{D}(\mathcal{R}){ }^{20}$ To control the overall complexity of these cells and (thereby, the number of the relevant cells of $\mathcal{D}_{\text {light }}$ that are met by $A_{K}$ ), we invoke the upper bound of Theorem 2.8 on the complexity of many cells in the hyperplane arrangement $\mathcal{A}(\mathcal{R})$.

\section{$4.3 \quad$ Piercing $\mathcal{F}$}

In this section we construct a net $N_{\mathcal{F}}$ for the sub-family $\mathcal{F} \subseteq \mathcal{K}_{\text {vcon. }}$. Recall that every set $K$ in $\mathcal{K}_{\text {vcon }}$ (and, in particular, in $\mathcal{F} \subseteq \mathcal{K}_{\text {vcon }}$ ) is traced in $\mathcal{D}_{z}(\mathcal{R})$ and, therefore, comes with a witness

\footnotetext{
${ }^{20}$ As is well known [10, the relative boundary of a $(d-1)$-dimensional convex set in $\mathbb{R}^{d}$ can determine a zone, within $\mathcal{A}(\mathcal{R})$, whose overall complexity is as large as $\Omega\left(r^{d-1}\right)$.
} 
hyperplane $H_{K} \in \mathcal{H}\left(\Pi_{K}^{\prime}\right)$, and a set $A_{K} \subseteq P_{K}$ of cardinality $\left|A_{K}\right| \geq C_{t r} \sigma^{\beta_{d-1}} \epsilon n$.

Denote $F_{K}:=A_{K} \cap\left(\bigcup \mathcal{D}_{\text {heavy }}\right)$. By the definition of $\mathcal{F}$, we have that

$$
\left|F_{K}\right| \geq \frac{\left|A_{K}\right|}{2} \geq \frac{C_{t r} \sigma^{\beta_{d-1}} \epsilon n}{2} .
$$

Also recall that there are at most $2 z$ heavy cells $\varphi$ in $\mathcal{D}_{\text {heavy }} \subseteq \mathcal{D}_{z}(\mathcal{R})$, and each of them encompasses a set $P_{\varphi}:=P \cap \varphi$ of at most $n / z$ points of $P$. For each of these heavy simplices $\varphi$ we construct a local net $N_{\varphi}$ for the recursive problem $\mathcal{K}\left(P_{\varphi}, \tilde{\epsilon}\right)$ where

$$
\tilde{\epsilon}:=\frac{\left(C_{t r} \sigma^{\beta_{d-1}} \epsilon n\right) m /(2 h I)}{n / z}=O\left(\frac{\sigma^{\beta_{d-1}}}{h \log r} \cdot \epsilon \cdot r\right)
$$

and define

$$
N_{\mathcal{F}}:=\bigcup_{\varphi \in \mathcal{D}_{\text {heavy }}} N_{\varphi}
$$

As a result, we obtain a net $N_{\mathcal{F}}$ of cardinality

$$
\left|N_{\mathcal{F}}\right| \leq 2 z \cdot f\left(\frac{\sigma^{\beta_{d-1} r}}{h \log r} \cdot \epsilon\right)
$$

Using the standard relations $\max \{1 / \sigma, h\} \lll z=o\left(r^{d}\right)$ and $r \lll s \lll 1 / \epsilon$, we obtain 21

$$
\left|N_{\mathcal{F}}\right| \leq 2 z \cdot f\left(\epsilon \cdot r^{1-\gamma^{\prime}}\right),
$$

where $\gamma^{\prime}>0$ is an arbitrary small, albeit fixed, constant.

Lemma 4.5. The net $N_{\mathcal{F}}$ pierces every convex set in $\mathcal{F}$.

Proof. It is enough to observe that for every $K \in \mathcal{F}$, at least $C_{t r} \sigma^{\beta_{d-1}} \epsilon n / 2$ of the points of $F_{K} \subseteq A_{K}$ are distributed over a subset $\mathcal{D}_{K} \subseteq \mathcal{D}_{\text {heavy }}$ of at most $h I / m$ heavy cells of $\mathcal{D}_{\text {heavy }}$ that are crossed by $H_{K}$. Hence, by the pigeonhole principle, there must be a heavy cell $\varphi \in \mathcal{D}_{K}$ whose set $P_{\varphi}$ includes at least $C_{t r} \sigma^{\beta_{d-1}} m \epsilon n /(2 h I)$ of the points of $F_{K}$. Since $\left|P_{\varphi}\right| \leq n / z$, the set $K$ must be $\tilde{\epsilon}$-heavy with respect to $P_{\varphi}$ and, therefore, pierced by $N_{\varphi} \subseteq N_{\mathcal{F}}$.

\subsection{Piercing $\mathcal{G}$}

For every $K \in \mathcal{G}$ denote

$$
G_{K}:=A_{K} \cap\left(\bigcup \mathcal{D}_{\text {light }}\right)
$$

Note that for every $K \in \mathcal{G}$ the subset $G_{K} \subseteq A_{K}$ is contained in the zone of the witness hyperplane $H_{K}$ within $\mathcal{D}_{\text {light }}$. By the definition of $\mathcal{G}$, we have that $\left|G_{K}\right| \geq C_{t r} \sigma^{\beta_{d-1}} \epsilon n / 2$ for every $K \in \mathcal{G}$. Also recall that every cell of $\mathcal{D}_{\text {light }}$ is a simplex that have been inherited by $\mathcal{D}_{z}(\mathcal{R})$ from the bottom-vertex triangulation $\mathcal{D}(\mathcal{R})$ of the arrangement $\mathcal{A}(\mathcal{R})$.

Definition. Denote $l:=\left\lceil\log r^{d} / z\right\rceil+1$. For each $1 \leq i \leq l$ denote $z_{i}:=2^{i-1} z$, and set

\footnotetext{
${ }^{21}$ Informally, this recursive term has "o(1/ $\left.\epsilon^{d}\right)$-influence" on the final bound as long as $z$ is substantially smaller than $r^{d}$. For the sake of brevity, we routinely omit the constant multiplicative factors within the recursive terms of the general form $a \cdot f(\epsilon \cdot b)$, provided that these factors are much larger than $1 / b$ and $1 / a$.
} 


$$
\mathcal{D}_{i}:=\left\{\varphi \in \mathcal{D}_{\text {light }}\left|n /\left(2 z_{i}\right)<\right| P_{\tau} \mid \leq n / z_{i}\right\}
$$

Note that we have $\left|\mathcal{D}_{i}\right| \leq 2 z_{i}=2^{i} z$ for every $1 \leq i \leq l$, and $\mathcal{D}_{\text {light }}=\biguplus_{i=1}^{l} \mathcal{D}_{i}$.

For every $1 \leq i \leq l$ let us denote $P_{i}:=P \cap\left(\bigcup \mathcal{D}_{i}\right)$, and let

$$
\mathcal{G}_{i}:=\left\{K \in \mathcal{G}|| G_{K} \cap P_{i}|\geq| G_{K} \mid / l\right\} .
$$

We obviously have $\mathcal{G}=\bigcup_{i=1}^{l} \mathcal{G}_{i}$, so it suffices a construct a separate net $N_{i}$ for each subfamily $\mathcal{G}_{i}$, for $1 \leq i \leq l$.

Piercing the families $\mathcal{G}_{i}$ - plan of operation. In contrast to the points of $F_{K}$ in Section 4.3 which were distributed over roughly $z / r$ cells of $\mathcal{D}_{\text {heavy }}$, the points of $G_{K} \cap P_{i}$ are potentially distributed over a potentially, far larger subset of cells within the collection $\mathcal{D}_{i} \subset \mathcal{D}_{\text {light }}$ whose overall cardinality is $O\left(z_{i}\right)=O\left(2^{i} \cdot z\right)$.

To localize a large fraction of $G_{K} \cap P_{i}$ in a much smaller number of cells, we at last use the vertical $\delta$-convexity of the sets $K \in \mathcal{G}_{i}$, along with the fact that $K$ is traced in $\mathcal{D}_{z}(\mathcal{R})$. Proposition 4.8 below yields a $(d-1)$-dimensional convex set $U_{K} \subset H_{K}$ so that $\Omega\left(\left|G_{K}\right| / l\right)$ of the points in $G_{K} \cap P_{i}$ come from the cells of $\mathcal{A}(\mathcal{R})$ that are crossed by the relative boundary $\partial U_{K}$ of $U_{K}$. By Theorem 2.7, the number of the latter cells of $\mathcal{A}(\mathcal{R})$ is at most $O\left(r^{d-2}\right)$.

However, some of these cells of $\mathcal{A}(\mathcal{R})$ may have fairly large complexities, in which case their bottom-vertex triangulations give rise to a far larger number of cells of $\mathcal{D}_{i}{ }^{22}$ Therefore, we first set aside the "high-complexity" cells $\Delta \in \mathcal{A}(\mathcal{R})$ (with their respective subsets $P_{i} \cap \Delta$ ), which yield at least $w_{i}$ simplicial cells of $\mathcal{D}(\mathcal{R})$. The threshold $w_{i}$ is determined in the sequel so as to guarantee, via Theorem 2.8, that the overall complexity of the "discarded" cells of $\mathcal{A}(\mathcal{R})$ is much smaller than $z_{i}$. Hence, the number of the points of $P_{i}$ that are set aside in this manner is substantially smaller than $n$.

Definition. Let $w>0$. We say that a cell $\Delta$ in the arrangement $\mathcal{A}(\mathcal{R})$ is $w$-complex if it contains at least $w$ simplices of the bottom-vertex triangulation $\mathcal{D}(\mathcal{R})$; otherwise, we say that the cell $\Delta$ is w-simple.

Proposition 4.6. The number of $w$-complex cells in $\mathcal{A}(\mathcal{R})$ is

$$
O\left(\frac{r^{d}(\log r)^{2 \eta_{d}}}{w^{2}}\right) .
$$

Proof. Let $m$ be the number of $w$-complex cells then the overall complexity of these cells is at least $m w$. Theorem 2.8 now yields

$$
m w=O\left(m^{1 / 2} r^{d / 2}(\log r)^{\eta_{d}}\right)
$$

or

$$
m=O\left(\frac{r^{d}(\log r)^{2 \eta_{d}}}{w^{2}}\right)
$$

\footnotetext{
${ }^{22}$ As a matter of fact, it is well known [10] that the relative boundary of a $(d-1)$-dimensional convex set $U \subset \mathbb{R}^{d-1}$ in $\mathcal{A}(\mathcal{R})$ may intersect cells of overall complexity $O\left(r^{d-1}\right)$. Hence, in absence of restrictions on the respective complexities of the cells of $\mathcal{A}(\mathcal{R})$ our argument may, at best, yield a naive " $O\left(1 / \epsilon^{d}\right)$-type" recursive term of the form $r^{d} \cdot f\left(\epsilon \cdot r^{d-1}\right)$.
} 
We fix a parameter $g>0$ so that $g=\Theta\left(l^{2}\right)$, and set

$$
\vartheta:=g / \sigma^{\beta_{d-1}}
$$

and

$$
w_{i}:=\frac{\vartheta r^{d}(\log r)^{2 \eta_{d}}}{z_{i}}=\frac{g r^{d}(\log r)^{2 \eta_{d}}}{\sigma^{\beta_{d-1}} z_{i}}
$$

Let $\mathcal{D}_{i}^{\prime}$ denote the subset of all the simplicial cells in $\mathcal{D}_{i}$ that arise from triangulating the $w_{i}$-complex cells of $\mathcal{A}(\mathcal{R})$.

Remark. Note that the assignments $z$ (and, therefore, also $z_{i}=z \cdot 2^{i-1}$ ) that are sufficiently close to $r^{d}$, yield fairly small complexity thresholds $w_{i}$. On the other hand, as the cut-off value $z$ remains substantially separated from $r^{d}$ (and below it), the recursive term on the right-hand side of (10) contributes only an "o(1/ $\left.\epsilon^{d}\right)$-type" term to the overall bound. Thus, our eventual choice of $z$ in Section 4.5 will seek to balance these two phenomena.

Proposition 4.7. We have that $\left|\mathcal{D}_{i}^{\prime}\right|=O\left(z_{i} / \vartheta\right)$.

Proof. By the preceding Proposition 4.6, there exist

$$
m_{i}=O\left(\frac{r^{d}}{w_{i}^{2}}(\log r)^{2 \eta_{d}}\right)
$$

$w_{i}$-complex cells in $\mathcal{A}(\mathcal{R})$. By Theorem 2.8 , the overall complexity of these cells is

$$
O\left(\frac{r^{d / 2}}{w_{i}} r^{d / 2}(\log r)^{2 \eta_{d}}\right)=O\left(\frac{r^{d / 2} \cdot z_{i}}{\vartheta r^{d}(\log r)^{2 \eta_{d}}} \cdot r^{d / 2}(\log r)^{2 \eta_{d}}\right)=O\left(z_{i} / \vartheta\right) .
$$

Clearly, the same asymptotic bound holds for the overall number of simplicial cells (including those of $\mathcal{D}_{i}^{\prime}$ ) that arise in the bottom-vertex triangulations of these cells of $\mathcal{A}(\mathcal{R})$.

Let $P_{i}^{\prime}:=P \cap\left(\bigcup \mathcal{D}_{i}^{\prime}\right)$. Since the $w_{i}$-complex cells of $\mathcal{A}(\mathcal{R})$ encompass at total of $O\left(z_{i} / \vartheta\right)$ light simplices of $\mathcal{D}_{i}$, and each of these simplices contains at most $n / z_{i}$ points of $P$, we have that $\left|P_{i}^{\prime}\right|=O(n / \vartheta)=O\left(\sigma^{\beta_{d-1}} n / g\right)$.

For each $1 \leq i \leq l$ let us denote

$$
\mathcal{G}_{i}^{\prime}:=\left\{K \in \mathcal{G}_{i}|| G_{K} \cap P_{i}^{\prime}|\geq| G_{K} \mid /(2 l)\right\} .
$$

Piercing the sets of $\mathcal{G}_{i}^{\prime}$. Note that, for every $K \in \mathcal{G}_{i}^{\prime}$, we have that $\left|G_{K} \cap P_{i}^{\prime}\right| \geq\left|G_{K}\right| /(2 l) \geq$ $\left|A_{K}\right| /(4 l) \geq C_{t r} \sigma^{\beta_{d-1}} \epsilon n /(4 l)$. Thus, every such set $K \in \mathcal{G}_{i}^{\prime}$ is $\Omega(g \epsilon / l)$-heavy with respect to the point set $P_{i}^{\prime}$. Therefore, each family $\mathcal{G}_{i}^{\prime}$ can be pierced by an auxiliary net $N_{i}^{\prime}$ of cardinality $f(g \epsilon / l)$ which we construct for the recursive problem $\mathcal{K}\left(P_{i}^{\prime}, D^{\prime} g \epsilon / l\right)$, where $D^{\prime}>0$ is a suitably small constant.

Notice that the overall cardinality of this nets satisfies

$$
\left|\bigcup N_{i}^{\prime}\right| \leq l \cdot f(g \epsilon / l)=l \cdot f(\epsilon \cdot l) .
$$

We add $\bigcup_{i=1}^{l} N_{i}^{\prime}$ to $N_{\mathcal{G}}$ and $N_{\text {vcon }}$ and, from now on, focus on piercing the remaining family $\mathcal{G}_{i} \backslash \mathcal{G}_{i}^{\prime}$ for every $1 \leq i \leq l$. For the sake of brevity, remove the sets of $\mathcal{G}_{i}^{\prime}$ from $\mathcal{G}_{i}$ (as they are already pierced by $\left.\bigcup_{i=1}^{l} N_{i}^{\prime}\right)$. 
Piercing the remaining sets of $\mathcal{G}_{i}$. For every remaining convex set $K \in \mathcal{G}_{i}$ let $\mathcal{D}_{K, i}$ denote the zone of $H_{K}$ in $\mathcal{D}_{i} \backslash \mathcal{D}_{i}^{\prime}$ (that is, the subset of all the simplices in $\mathcal{D}_{i} \backslash \mathcal{D}_{i}^{\prime}$ that are crossed by the witness hyperplane $H_{K}$ of $\left.K\right)$. Let us denote $G_{K, i}:=G_{K} \cap\left(\bigcup \mathcal{D}_{K, i}\right)$ for every $K \in \mathcal{G}_{i}$. By the definition of $\mathcal{G}_{i}$ (and the prior removal of $\mathcal{G}_{i}^{\prime}$ ), we have that for every $K \in \mathcal{G}_{i}$

$$
\left|G_{K, i}\right| \geq\left|G_{K}\right| /(2 l) \geq\left|A_{K}\right| /(4 l) \geq C_{t r} \sigma^{\beta_{d-1}} \epsilon n /(4 l) .
$$

Recall that for each vertically $\delta$-convex set $K \in \mathcal{K}_{\text {vcon }}$, the set $\left(\begin{array}{c}P_{K} \\ d+1\end{array}\right)$ contains at least $(1-\delta)\left(\begin{array}{l}\lceil\epsilon n\rceil \\ d+1\end{array}\right)$ vertically convex $(d+1)$-subsets, where $\delta:=C_{\mathrm{vcon}} / r^{d(d+1)+1}$.

Proposition 4.8. There exists a choice of $C_{\mathrm{vcon}}>0$ in (3) so the the following property holds:

For every $1 \leq i \leq l$, and every remaining set $K \in \mathcal{G}_{i}$ there is a $(d-1)$-dimensional convex set $U_{K}$ within $H_{K}$, and a subset $\tilde{G}_{K, i} \subseteq G_{K, i}$ of cardinality at least $\left|G_{K, i}\right| / 3$ that entirely lies in (the union of the open cells of) the zone of $\partial U_{K}$ within $\mathcal{A}(\mathcal{R})$.

Proof. Fix $K \in \mathcal{G}_{i}$. For every simplicial cell $\varphi \in \mathcal{D}_{K, i}$ let us denote $G_{K, \varphi}:=G_{K, i} \cap \varphi$. We apply Lemma 2.4 to the resulting partition

$$
G_{K, i}=\biguplus_{\varphi \in \mathcal{D}_{K, i}} G_{K, \varphi}
$$

of the point set $G_{K, i}$ into $k=\left|\mathcal{D}_{K, i}\right|=O\left(r^{d}\right)$ subsets. Then a random $k^{\prime}$-sample $S \subseteq G_{K, i}$, for $k^{\prime}=\lceil C k\rceil$ (and with the constant $C>0$ as prescribed in the lemma), meets the following condition with probability at least $1 / 2$ :

(C1) The parts $G_{K, \varphi}$ that are intersected by $S$, encompass a subset $\tilde{G}_{K, i} \subseteq G_{K, i}$ of at least $\left|G_{K, i}\right| / 3$ points.

On the other hand, since $K$ is vertically $\delta$-convex (as $\left.\mathcal{G}_{i} \subseteq \mathcal{G} \subseteq \mathcal{K}_{\mathrm{vcon}}\right)$, there exist at most $\delta\left(\begin{array}{l}\lceil\epsilon n\rceil \\ d+1\end{array}\right)$ $(d+1)$-subsets in $\left(\begin{array}{c}G_{K, i} \\ d+1\end{array}\right)$ that are not vertically convex. Therefore, and since $\left|G_{K, i}\right|=\Omega\left(\sigma^{\beta_{d-1}} \epsilon n / l\right)$, $G_{K, i}$ must be vertically $\tilde{\delta}$-convex, for

$$
\tilde{\delta}=O\left(\delta \cdot \frac{l^{d+1}}{\sigma^{(d+1) \beta_{d-1}}}\right) .
$$

Since $\sigma, l \lll r$, we have that $\tilde{\delta}=o\left(1 / r^{d(d+1)}\right)=O\left(1 / k^{d+1}\right)$, Lemma 2.5 implies (given a suitable constant of proportionality $C_{\mathrm{vcon}}>0$ in the definition (3) of $\delta$ ) that the sample $S$ is vertically convex with probability at least 0.4 while $(\mathrm{C} 1)$ is still satisfied. See Figure 13 .

Assume then that our sample $S$ is vertically convex and satisfies (C1). Since $K$ is traced in $\mathcal{D}_{z}(\mathcal{R})$, and $S \subseteq G_{K, i} \subseteq A_{K}$, the vertical projection $(p)_{H_{K}}$ of every point $p \in S$ onto $H_{K}$ lies in the same cell of $\mathcal{A}(\mathcal{R})$ as $p$. Let $(S)_{H_{K}}:=\left\{(p)_{H_{K}} \mid p \in S\right\}$. Note that we have that $(S)_{H_{K}} \subset H_{K}$, so the convex hull $U_{K}=\operatorname{conv}\left((S)_{H_{K}}\right)$ is a $(d-1)$-dimensional convex set, within the hyperplane $H_{K}$. Furthermore, by the vertical convexity of $S$, for every cell $\Delta$ in $\mathcal{A}(\mathcal{R})$ that contains a point $p \in S$, the projected point $(p)_{H_{K}} \in \Delta$ that lies on the relative boundary $\partial U_{K}$ of $U_{K}$, and within the same cell $\Delta$. Therefore, condition (C1) yields at least $\left|G_{K, i}\right| / 3$ points of $\tilde{G}_{K, i}$ which lie in the light simplices $\varphi \in \mathcal{D}_{K, i}$ whose ambient cells $\Delta \supset \varphi$ in the arrangement $\mathcal{A}(\mathcal{R})$ meet $\partial U_{K}$.

Theorem 2.7 implies the following property of the the sub-sets $\tilde{G}_{K, i}$ that were obtained through Proposition 4.8, for $K \in \mathcal{G}_{i}$. 


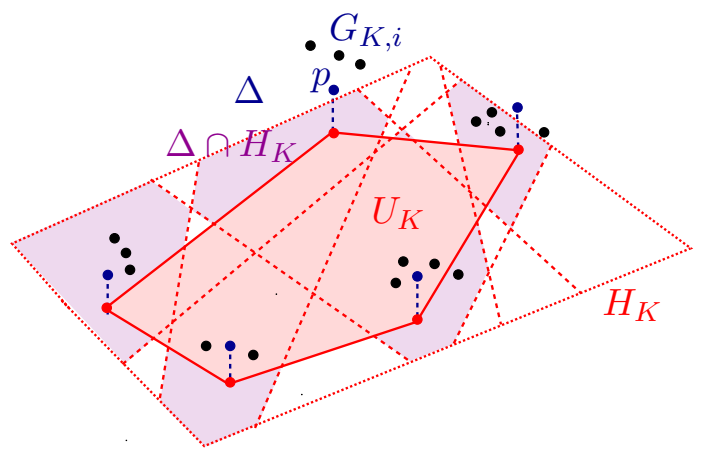

Figure 13: Proof of Proposition 4.8. We sample a subset $S$ of $\lceil C k\rceil$ points (blue) in $G_{K, i}$. The set $S$ is in a vertically convex position. The projected points of $(S)_{H_{K}}$ are drawn red, and determine the $(d-1)$ dimensional convex polytope $U_{K}:=\operatorname{conv}\left((S)_{H_{K}}\right)$ within the hyperplane $H_{K}$. The cross-sections $\Delta \cap H_{K}$ of the ambient cells $\Delta \in \mathcal{A}(\mathcal{R})$ of the points $p \in S$ are drawn purple.

Corollary 4.9. Let $K$ be a remaining set of $\mathcal{G}_{i}$ with the subset $\tilde{G}_{K, i}$ as in Proposition 4.8. Then there exists a subset $\tilde{\mathcal{D}}_{K, i} \subseteq \mathcal{D}_{K, i}$ of $O\left(w_{i} r^{d-2}\right)$ light simplicial cells in $\mathcal{D}_{K, i}$ whose union covers $\tilde{G}_{K, i}$.

Proof. Fix a convex set $K \in \mathcal{G}_{i}$. Proposition 4.8 yields a convex set $U_{K} \subset H_{K}$ whose zone in $\mathcal{A}(\mathcal{R})$ encompasses $\mathcal{G}_{K, i}$. Thus, $\tilde{\mathcal{D}}_{K, i}$ is defined as the set of all the light simplicial cells in $\mathcal{D}_{K, i}$ that arise from triangulating the $w_{i}$-simple cells $\Delta$ in the zone of the relative boundary $\partial U_{K}$ of $U_{K}$ within $\mathcal{A}(\mathcal{R})$. It, therefore, remains to check that the cardinality $\tilde{\mathcal{D}}_{K, i}$ meets the asserted upper bound.

Indeed, note that every cell $\Delta$ of $\mathcal{A}(\mathcal{R})$ in the zone of $\partial U_{K}$ determines a unique cell $\Delta^{\prime}=\Delta \cap H_{K}$ in the $(d-1)$-dimensional arrangement of the collection $\mathcal{R}^{\prime}=\left\{H \cap H_{K} \mid H \in \mathcal{R}\right\}$ of $(d-2)$-planes within $H_{K}=\mathbb{R}^{d-1}$; this cell $\Delta^{\prime}$ too belongs to the zone of $\partial U_{K}$, now within a $(d-1)$-dimensional arrangement $\mathcal{A}\left(\mathcal{R}^{\prime}\right)$ (again, see Figure 13). By Theorem 2.7, the zone of $\partial U_{K}$ within $\mathcal{A}\left(\mathcal{R}^{\prime}\right)$ is comprised of $O\left(r^{d-2}\right)$ cells $\Delta^{\prime}$, which also bounds the number of $d$-dimensional cells in the zone of $U_{K}$ within $\mathcal{A}(\mathcal{R})$. Furthermore, any $w_{i}$-simple cell in this zone contributes at most $w_{i}$ light simplicial cells to the set $\tilde{\mathcal{D}}_{K, i}$, for a total of $O\left(w_{i} r^{d-2}\right)$ such light simplicial cells.

The nets $N_{i}$ for $\mathcal{G}_{i}$. We are now ready to describe the nets $N_{i}$ for the remaining families $\mathcal{G}_{i}$, for every $1 \leq i \leq l$. For every simplicial cell $\varphi \in \mathcal{D}_{i} \backslash \mathcal{D}_{i}^{\prime}$ we construct, and add to $N_{i}$, a net $N_{\varphi}$ for the recursive problem $\mathcal{K}\left(P_{\varphi}, \tilde{\epsilon}_{i}\right)$, where

$$
\tilde{\epsilon}_{i}:=\tilde{C} \cdot \frac{\sigma^{\beta_{d-1}} \epsilon n /\left(l w_{i} r^{d-2}\right)}{n / z_{i}}=\Theta\left(\frac{\sigma^{\beta_{d-1}} z_{i} \epsilon}{w_{i} r^{d-2} l}\right)=\Theta\left(\frac{\sigma^{2 \beta_{d-1}} z_{i}^{2} \epsilon}{g(\log r)^{2 \eta_{d}} r^{2 d-2} l}\right),
$$

where $\tilde{C}>0$ is a suitably small constant to be determined shortly.

Note that the last equality uses (11). Due to our choice of parameters $l=O(\log 1 / \epsilon), g=$ $\Theta\left(l^{2}\right)=O\left(\log ^{2}(1 / \epsilon)\right)$ and $1 / \sigma \lll r \lll z_{i}$, the right part of 113$)$ is bounded from below by $\epsilon z_{i}^{2} / r^{2 d-2+\gamma^{\prime}}$ for any arbitrary small, albeit fixed, constant $\gamma^{\prime}>0$. Hence, the overall cardinality of this net

$$
N_{i}:=\bigcup_{\varphi \in \mathcal{D}_{i} \backslash \mathcal{D}_{i}^{\prime}} N_{\varphi}
$$

is at most

$$
\left|N_{i}\right|=O\left(z_{i} \cdot f\left(\epsilon \cdot \frac{z_{i}^{2}}{r^{2 d-2}} \cdot \frac{\sigma^{2 \beta_{d-1}}}{g(\log r)^{2 \eta_{d}}}\right)\right)=O\left(z_{i} \cdot f\left(\epsilon \cdot \frac{z_{i}^{2}}{r^{2 d-2+\gamma^{\prime}}}\right)\right)
$$


where $\gamma^{\prime}>0$ is an arbitrary small constant.

Proposition 4.10. There exists a choice of the constant $\tilde{C}>0$ in 13 so that every remaining set $K \in \mathcal{G}_{i}$ is intersected by the net $N_{i}$, for all $1 \leq i \leq l$.

Proof. Fix a set $K \in \mathcal{G}_{i}$. Proposition 4.8 yields a subset $\tilde{G}_{K, i} \subseteq P_{K}$ of $\Omega\left(\sigma^{\beta_{d-1}} \epsilon n / l\right)$ points which, according to Corollary 4.9 , are distributed over $O\left(w_{i} r^{d-2}\right)$ light simplices of $\tilde{\mathcal{D}}_{K, i}$. By the pigeonhole principle, there must be a simplicial cell $\varphi \in \tilde{D}_{K, i}$ that satisfies

$$
\left|\tilde{G}_{K, i} \cap \varphi\right|=\Omega\left(\frac{\left|\tilde{G}_{K, i}\right|}{w_{i} r^{d-2}}\right)=\Omega\left(\frac{\sigma^{\beta_{d-1}} \epsilon n}{l w_{i} r^{d-2}}\right) .
$$

Since every light simplicial cell $\varphi \in \mathcal{D}_{K, i} \subset \mathcal{D}_{i}$ satisfies $\left|P_{\varphi}\right| \leq n / z_{i}$, there is a constant $\tilde{C}>0$ (which does not depend on $K$ ) so that $K$ is $\tilde{\epsilon}_{i}$-heavy with respect to $P_{\varphi}$ and, thereby, is hit by the local net $N_{\varphi} \subseteq N_{i}$.

We, therefore, choose $\tilde{C}>0$ in 13 so as to meet the criterion of Proposition 4.10. Hence, our net $N_{\mathcal{G}}$ for $\mathcal{G}$ is duly defined as the union of the previously defined nets, namely,

$$
N_{\mathcal{G}}:=\left(\bigcup_{1 \leq i \leq l} N_{i}^{\prime}\right) \cup\left(\bigcup_{1 \leq i \leq l} N_{i}\right) .
$$

Combining Proposition 4.10 with the bounds $(12)$ and (13), we obtain the following result.

Theorem 4.11. The net $N_{\mathcal{G}}$ is a transversal for $\mathcal{G}$. Furthermore, we have that

$$
\left|N_{\mathcal{G}}\right| \leq l \cdot f(\epsilon \cdot l)+\sum_{i=1}^{l} z_{i} f\left(\epsilon z_{i}^{2} / r^{2 d-2+\gamma^{\prime}}\right),
$$

where $\gamma^{\prime}>0$ is an arbitrary small constant.

\subsection{Piercing the vertically convex sets: Wrap-up}

We now put together the nets of the previous Sections 4.1, 4.2, 4.3, and 4.4, we obtain a net

$$
N_{\text {vcon }}:=N_{\geq h} \cup N\left(\epsilon^{\prime}, s\right) \cup N_{t r} \cup N_{\mathcal{F}} \cup N_{\mathcal{G}} .
$$

Namely, in Section 4.1 we obtained the net $N_{\geq h}$ whose cardinality satisfies

$$
N_{\geq h} \leq f(\epsilon, \lambda / h, \sigma / 2),
$$

and that pierces every convex set in the sub-family $\mathcal{K}_{\geq h}=\mathcal{K}\left(P, \Pi_{\geq h}, \epsilon, \sigma / 2\right)$.

In Section 4.2 we first constructed the net $N\left(\epsilon^{\prime}, s\right)$, whose cardinality (according to (7)) is

$$
\left|N\left(\epsilon^{\prime}, s\right)\right|=O\left(s \cdot f\left(\epsilon \cdot s^{1 /(d-1)} \cdot \frac{\cdot C^{\prime} \sigma^{\beta_{d-1}}}{r}\right)\right),
$$

and that pierces every convex set that in $\epsilon^{\prime}$-narrow in the vertical simplicial partition $\mathcal{P}(s)$. We then constructed a net $N_{t r}$ whose cardinality (according to Proposition 4.2 ) is

$$
\left|N_{t r}\right| \leq O\left(\frac{s^{2(d-1)^{2}} r}{\sigma^{\beta_{d-1}} \epsilon^{d-1}}\right)
$$


Furthermore, according to Theorem 4.3 , the net $N_{t r}$ pierces every remaining, $\epsilon^{\prime}$-spread convex set $K \in \mathcal{K}_{\text {vcon }}$ that is not traced in the decomposition $\mathcal{D}_{z}(\mathcal{R})$ (and that is not pierced by the previous nets $N_{\geq h}$ and $\left.N\left(\epsilon^{\prime}, s\right)\right)$.

By the end of Section 4.2 , every remaining set $K \in \mathcal{K}_{\mathrm{vcon}}$ was traced $\mathcal{D}_{z}(\mathcal{R})$. In particular, it was equipped with a relatively large witness sub-set $A_{K} \subseteq P_{K}$ whose points lied in the zone of the witness hyperplane $H_{K}$, within the decomposition $\mathcal{D}_{z}(\mathcal{R})$. These sets were further sub-divided into the families $\mathcal{F}$ and $\mathcal{G}$ based on the respective distributions of the points of $A_{K}$ amongst the cells of $\mathcal{D}_{z}(\mathcal{R})$.

Finally, in Sections 4.3 and 4.4 we established, respectively, Lemma 4.5 and Theorem 4.11 , which yield nets $N_{\mathcal{F}}$ and $N_{\mathcal{G}}$ for the respective families $\mathcal{F}$ and $\mathcal{G}$. Combining (10) and (15), we obtain that the overall cardinality of $N_{\mathcal{F}} \cup N_{\mathcal{G}}$ satisfies

$$
\left|N_{\mathcal{F}} \cup N_{\mathcal{G}}\right| \leq 2 z \cdot f\left(\epsilon \cdot r^{1-\gamma^{\prime}}\right)+l \cdot f(\epsilon \cdot l)+\sum_{i=1}^{l} z_{i} \cdot f\left(\epsilon \cdot \frac{z_{i}^{2}}{r^{2 d-2+\gamma^{\prime}}}\right),
$$

where $\gamma^{\prime}>0$ is an arbitrary small, albeit fixed, constant. Moreover, using the relations $1 / \sigma \lll$ $r \ll s \lll 1 / \epsilon$ yields that the overall cardinality of the above nets $N\left(\epsilon^{\prime}, s\right)$ and $N_{t r}$ satisfies

$$
\left|N\left(\epsilon^{\prime}, s\right) \cup N_{t r}\right| \leq s \cdot f\left(\epsilon \cdot s^{1 /(d-1)-\gamma^{\prime}}\right)+O\left(\frac{1}{\epsilon^{d-1+\gamma^{\prime}}}\right),
$$

where $\gamma^{\prime}>0$ is again an arbitrary small constant.

Combining (17) and (18) with the upper bound $f(\epsilon, \lambda / h, \sigma / 2)$ on $\left|N_{\geq h}\right|$, we obtain

$$
\begin{aligned}
& \left|N_{\mathrm{vcon}}\right| \leq f(\epsilon, \lambda / t, \sigma / 2)+s \cdot f\left(\epsilon \cdot s^{1 /(d-1)-\gamma^{\prime}}\right)+O\left(\frac{1}{\epsilon^{d-1+\gamma^{\prime}}}\right)+ \\
& \quad+2 z \cdot f\left(\epsilon \cdot r^{1-\gamma^{\prime}}\right)+l \cdot f(\epsilon \cdot l)+\sum_{i=1}^{l} z_{i} \cdot f\left(\epsilon \cdot \frac{z_{i}^{2}}{r^{2 d-2+\gamma^{\prime}}}\right)
\end{aligned}
$$

where $\gamma^{\prime}>0$ is an arbitrary small constant.

Choosing $z=\Theta\left(r^{d-1 / 2}\right)$ yields the following result.

Theorem 4.12. The net $N_{\mathrm{vcon}}$ in (16) pierces every set in the family $\mathcal{K}_{\mathrm{vcon}}$, which encompasses all the vertically $\delta$-convex sets in $\mathcal{K}(\bar{P}, \Pi, \epsilon, \sigma)$. Furthermore, we have that

$$
\begin{gathered}
\left|N_{\mathrm{vcon}}\right| \leq f(\epsilon, \rho / h, \sigma / 2)+s \cdot f\left(\epsilon \cdot s^{1 /(d-1)-\gamma^{\prime}}\right)+ \\
+O\left(l \cdot f(\epsilon \cdot l)+\sum_{i=0}^{l} 2^{i} \cdot r^{d-1 / 2} \cdot f\left(\epsilon \cdot 2^{2 i} \cdot r^{1-\gamma^{\prime}}\right)\right)+O\left(\frac{1}{\epsilon^{d-1+\gamma^{\prime}}}\right),
\end{gathered}
$$

where $h, r$ and $s$ are arbitrary small, albeit fixed, degrees of $1 / \epsilon$ that satisfy $h \lll r \lll s \lll 1 / \epsilon$, $\Theta(\log 1 / \epsilon)=l \leq\left\lceil\frac{1}{2} \log r\right\rceil+1$, and $\gamma^{\prime}>0$ is an arbitrary small constant.

Remark. Notice that, except for the first term $f(\epsilon, \rho / h, \sigma / 2)$, all the terms in 20 are either outright $o\left(1 / \epsilon^{d}\right)$, or of the general form $q^{a} \cdot f\left(\epsilon \cdot q^{b}\right)$, where $a$ and $b$ are positive constants that satisfy $a / b<d$. In other words, except for its first term (which involves a recurrence in the density $\rho)$, the bound is distinctly of the "o $\left(1 / \epsilon^{d}\right)$-type". 


\section{$5 \quad$ Piercing the sets of $\mathcal{K}_{\text {surr }}$}

In this section we construct a net $N_{\text {surr }}$ for the sub-family $\mathcal{K}_{\text {surr }}:=\mathcal{K}(P, \Pi, \epsilon, \sigma) \backslash \mathcal{K}_{\text {vcon }}$. For each

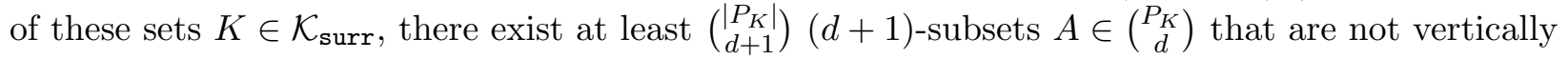
convex.

Similar to the previous case of $\mathcal{K}_{\mathrm{vcon}}$, we initialize $N_{\text {surr }}:=\emptyset$, and proceed through a sequence of more elementary steps ${ }^{23}$ At each step we construct, and immediately add to $N_{\text {surr }}$, a net which pierces a certain sub-family of the remaining sets of $\mathcal{K}_{\text {surr }}$ (that were not previously pierced by $\left.N_{\text {surr }}\right)$. Once a set $K \in \mathcal{K}_{\text {surr }}$ is pierced by $N_{\text {surr }}$, it is immediately removed from further consideration in the following stages, whereas its surviving counterparts in $\mathcal{K}_{\text {surr }}$ meet a more refined set of criteria, which facilitate the ultimate disposal of such sets using the paradigm sketched in Section 2.2.

\subsection{Characteristic $(d+1)$-tuples}

We invoke the vertical simplicial $s$-partition $\mathcal{P}(s)=\left\{\left(P_{1}, \Delta_{1}\right), \ldots,\left(P_{s}, \Delta_{s}\right)\right\}$ of $P$ as described in Section 3.1 (and identical to the partition that was used in Section 4.2). The partition parameter $s$ is proportional to an arbitrary small, albeit fixed degree of $1 / \epsilon$ that satisfies $s \ggg 1 / \delta$. Similar to Section 3.4, for every $p \in P$ we use $\Delta(p)$ to denote the ambient simplex $\Delta_{i}$ of $p$ in the present vertical partition $\mathcal{P}(s)$ (so that each point $p \in P_{i}$ lies in the vertical prism over the simplex $\Delta_{i} \subset \mathbb{R}^{d-1}$ ).

Regular $(d+1)$-tuples. Notice that every $K \in \mathcal{K}_{\text {surr }}$ determines at least $\left(\begin{array}{c}\left|P_{K}\right| \\ d\end{array}\right)(d+1)$-sets $\left\{p_{1}, \ldots, p_{d+1}\right\} \in\left(\begin{array}{c}P_{K} \\ d+1\end{array}\right)$ so that $p_{d+1}^{\perp} \in \operatorname{conv}\left(p_{1}^{\perp}, \ldots, p_{d}^{\perp}\right)$. Recent studies of the regularity properties of geometrically defined hypergraphs [30, 31] yield a so called homogeneous $(d+1)$-tuple $P_{1}^{\prime}, \ldots, P_{d+1}^{\prime} \subset P_{K}$, so that each set $P_{i}^{\prime}$ has cardinality $\Omega\left(\delta^{\Omega(1)}\left|P_{K}\right|\right)$, and the following Turántype condition is satisfied: For each $\left(p_{1}, p_{2}, \ldots, p_{d+1}\right) \in P_{1}^{\prime} \times P_{2}^{\prime} \times \ldots \times P_{d+1}^{\prime}$ we have that $p_{d+1}^{\perp} \in \operatorname{conv}\left(p_{1}^{\perp}, \ldots, p_{d}^{\perp}\right)$. In particular, the order type of $\left(p_{1}^{\perp}, \ldots, p_{d+1}^{\perp}\right)$ is invariant in the choice of the representatives $p_{i} \in P_{i}^{\prime}$, for $1 \leq i \leq d+1$.

Below we argue that, if the projected points of $P_{K}^{\perp}$ are reasonably well-spread with respect to $\mathcal{P}(s)$, the desired regular $(d+1)$-tuple within $P_{K}$ is, in fact, cut out by the sets of $\mathcal{P}(s)$. As in Section 4 , the narrow sets can be dispatched using Lemma 3.1 .

The net $N\left(\epsilon_{\delta}, s\right)$. Denote

$$
\epsilon_{\delta}:=\frac{\delta \epsilon}{32 d^{2}(d+1)\left(\begin{array}{c}
d-1) d \\
d-1
\end{array}\right)} .
$$

Then Lemma 3.1 yields an auxiliary net $N\left(\epsilon_{\delta}, s\right)$ of cardinality

$$
\left|N\left(\epsilon_{\delta}, s\right)\right|=O\left(s \cdot f\left(\epsilon_{\delta} \cdot s^{1 /(d-1)}\right)\right)=O\left(s \cdot f\left(\epsilon \cdot \delta s^{1 /(d-1)}\right)\right)
$$

that pierces every $\epsilon_{\delta}$-narrow set in $\mathcal{K}_{\text {surr }}$. We add $N\left(\epsilon_{\delta}, s\right)$ to $N_{\text {surr }}$ and remove from $\mathcal{K}_{\text {surr }}$ every set that is $\epsilon_{\delta}$-narrow in $\mathcal{P}(s)$ (for its is already pierced by $N_{\text {surr }}$ ).

Definition. Let $\Delta_{j_{1}}, \ldots, \Delta_{j_{d+1}}$ be $d+1$ ambient simplices of some $d+1$ subsets $P_{j_{i}}$ within the vertical partition $\mathcal{P}(s)$ (so that $P_{j_{i}}^{\perp} \subset \Delta_{j_{i}}$ ), for $1 \leq i \leq d+1$. We say that $\Delta_{j_{d+1}}$ is surrounded by $\Delta_{j_{1}}, \ldots, \Delta_{j_{d}}$ if for every choice $x_{i} \in \Delta_{j_{i}}$, for $1 \leq i \leq d+1$, we have that $x_{d+1} \in \operatorname{conv}\left(x_{1}, \ldots, x_{d}\right)$.

\footnotetext{
${ }^{23}$ Note, though, that the set $\Pi$ will play no role in the construction of $N_{\text {surr }}$, so our recursive bound for the cardinality of $N_{\text {surr }}$ will not involve any terms that depend on the density $\rho=|\Pi| /\left(\begin{array}{l}n \\ d\end{array}\right)$.
} 

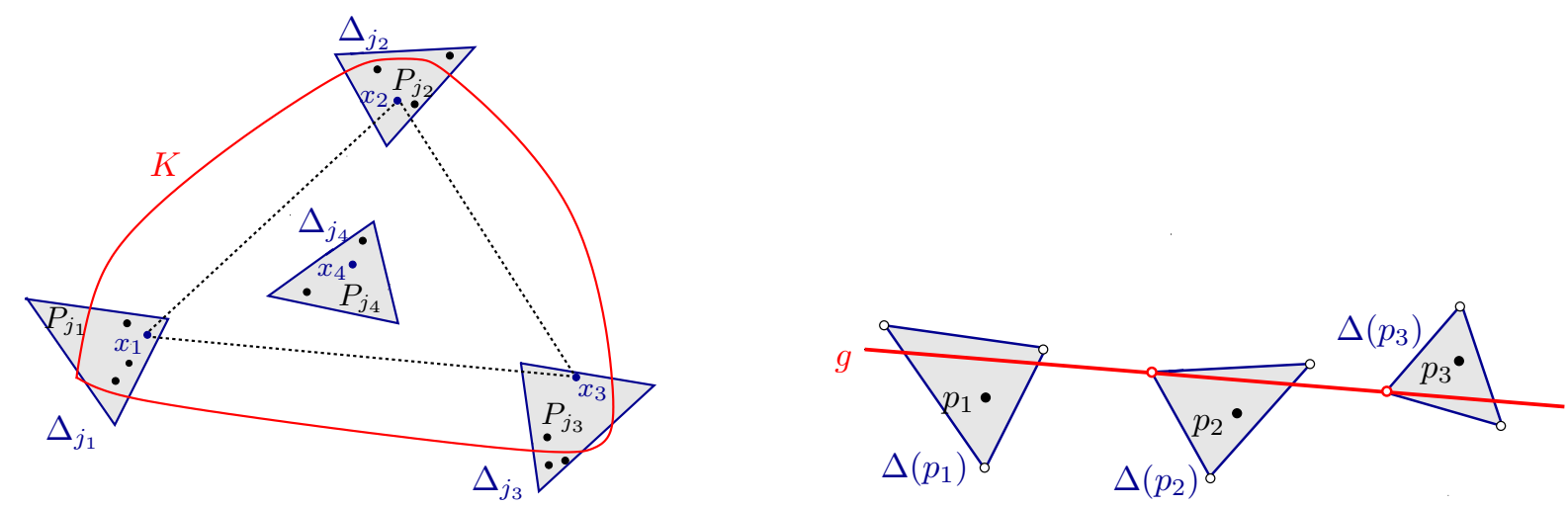

Figure 14: Left - The characteristic $(d+1)$-tuple (view in dimension $d=3$, in the 2-dimensional projection). The simplex $\Delta_{j_{4}}$ is surrounded by the simplices $\Delta_{j_{1}}, \Delta_{j_{2}}$, and $\Delta_{j_{3}}$ : for each selection $x_{i} \in \Delta_{j_{i}}$, where $1 \leq i \leq$ 4, we have that $x_{4} \in \operatorname{conv}\left(x_{1}, x_{2}, x_{3}\right)$. Right - Proof of Claim 5.2. A degenerate 3-set $A=\left\{p_{1}, p_{2}, p_{3}\right\} \in \Upsilon_{K}$ in dimension $d=3$ (view in the vertical projection). The line $g$ crosses all 3 ambient simplices $\Delta\left(p_{i}\right)$, and passes through the vertices of $\Delta\left(p_{2}\right)$ and $\Delta\left(p_{3}\right)$.

Remark. Note that, according to this definition, the point $x_{i}$ need not necessarily come from the projection of $P_{j_{i}}$. Also note that, in order for $\Delta_{j_{d+1}}$ to be surrounded, the $d+1$ simplices $\Delta_{j_{i}}$ (and, thereby, also their respective sets $\left.P_{j_{i}}\right)$ need to be distinct and pairwise disjoint.

Let $\mathcal{T}=\left(P_{j_{1}}, \ldots, P_{j_{d+1}}\right)$ be an ordered $(d+1)$-tuple of the sub-sets $P_{j_{i}} \in \mathcal{P}(s)$, for $1 \leq i \leq d+1$. We say that $\mathcal{T}$ is characteristic for the convex set $K \in \mathcal{K}_{\text {surr }}$ if the following conditions are met (see Figure 14(left)):

1. The ambient simplex $\Delta_{j_{d+1}}$ of $P_{j_{d+1}}$ is surrounded by the respective ambient simplices $\Delta_{j_{i}}$ of the remaining $d$ sets $P_{j_{i}}$, for $1 \leq i \leq d$.

2. We have that

$$
\left|P_{K} \cap P_{j_{i}}\right| \geq C_{\text {surr }} \cdot \delta\left|P_{K}\right| / s
$$

for all $1 \leq i \leq d+1$. Here $C_{\text {surr }}>0$ is a constant to be determined in the sequel.

Theorem 5.1. The following property holds for any small enough constant assignment $C_{\text {surr }}>0$ in (22):

For every $\epsilon_{\delta}$-spread convex set $K \in \mathcal{K}_{\text {surr }}$ there exists at least one characteristic $(d+1)$-tuple $\mathcal{T}=\left(P_{j_{1}}, \ldots, P_{j_{d+1}}\right)$ as above.

Proof. We establish the theorem with $C_{\text {surr }}=1 /(4 d+4)$. Let $k \geq d$. We say that a collection of $k$ simplices $\left\{\Delta_{i_{1}}, \ldots, \Delta_{i_{k}}\right\}$ in $\mathbb{R}^{d-1}$, for $1 \leq j_{1} \neq j_{2} \neq \ldots \neq j_{k} \leq s$, is called regular if no $d$ of its members can be crossed by a single $(d-2)$-plane in $\mathbb{R}^{d-1}$. (The regularity of $\left\{\Delta_{i_{1}}, \ldots, \Delta_{i_{k}}\right\}$ is equivalent to the following property: every $k$ representatives $x_{1} \in \Delta_{i_{1}}, \ldots, x_{k} \in \Delta_{i_{k}}$ are in a general position within $\mathbb{R}^{d-1}$, and their order type is invariant in the choice of $x_{j} \in \Delta_{i_{j}}$; see, e.g., [30.)

Accordingly, we say that a $k$-set of points $\left\{p_{1}, \ldots, p_{k}\right\} \in\left(\begin{array}{c}P_{K} \\ k\end{array}\right)$ is regular if their ambient simplices $\Delta\left(p_{i}\right)$ in the vertical partition $\mathcal{P}(s)$ are distinct, and form a regular collection $\left\{\Delta\left(p_{1}\right), \ldots, \Delta\left(p_{k}\right)\right\}$. Otherwise, we say that the $k$-set $\left\{p_{1}, \ldots, p_{k}\right\}$ is degenerate. Our proof of Theorem 5.1 relies on the following property. 
Claim 5.2. Let $K \in \mathcal{K}_{\text {surr }}$. Then there exist at most $\left(\delta /(4(d+1))\left(\begin{array}{c}\left|P_{K}\right| \\ d\end{array}\right)\right.$ degenerate $d$-subsets in $\left(\begin{array}{c}P_{K} \\ d\end{array}\right)$. Hence, there exist at most $(\delta / 4)\left(\begin{array}{c}\left|P_{K}\right| \\ d+1\end{array}\right)$ degenerate $(d+1)$-subsets in $\left(\begin{array}{c}P_{K} \\ d+1\end{array}\right)$.

Proof Claim 5.2. Since any vertically degenerate $d$-set contributes to at most $\left|P_{K}\right|-d$ vertically degenerate $(d+1)$-sets, it is immediate to check that the second property follows from the first one. Assume for a contradiction that there exist at least $\frac{\delta}{4(d+1)}\left(\begin{array}{c}P_{K} \\ d\end{array}\right)$ degenerate subsets in $\left(\begin{array}{c}P_{K} \\ d\end{array}\right)$.

Let $\Upsilon_{K} \subseteq\left(\begin{array}{c}P_{K} \\ d\end{array}\right)$ denote the subset of all the degenerate $d$-sets in $\left(\begin{array}{c}P_{K} \\ d\end{array}\right)$. Fix any $A \in \Upsilon_{K}$. Then there must be a $(d-2)$-plane $g$ within $\mathbb{R}^{d-1}$ that crosses every simplex in the respective multicollection $\Sigma:=\left\{\Delta\left(p_{1}\right), \ldots, \Delta\left(p_{d}\right)\right\}$. In particular, if $\Sigma$ is a multiset so that $\Delta\left(p_{j}\right)=\Delta\left(p_{j^{\prime}}\right)$ for some $1 \leq j \neq j^{\prime} \leq d$, then the $(d-2)$-plane $g$ through $A^{\perp} \backslash\left\{p_{i}^{\perp}\right\}$ meets every simplex in $\Sigma$.

By Lemma 3.4, there must be a $(d-1)$-subset $B \in\left(\begin{array}{c}A \\ d-1\end{array}\right)$, and a $(d-2)$-plane transversal $g$ to $\Sigma$ that passes through some $d-1$ vertices of the simplices from $\{\Delta(p) \mid p \in B\}$; see Figure 14 (right). We thus label $A$ with such a pair $(B, g)$. We apply the above labelling to all the (at least) $\frac{\delta}{4(d+1)}\left(\begin{array}{c}\lceil\epsilon n\rceil \\ d\end{array}\right)$ such $d$-sets $A \in \Upsilon_{K}$. Since any $(d-1)$-set $B \in\left(\begin{array}{c}P_{K} \\ d-1\end{array}\right)$ participates in at most $\left(\begin{array}{c}(d-1) d \\ d-1\end{array}\right)$ of the labels $(B, g)$, by the pigeonhole principle there must be a label $(B, g)$ that is shared by at least $\epsilon_{\delta} n$ of the $d$-sets $A \in \Upsilon_{K}$. The desired contradiction follows because each of these $d$-sets $A$ contributes a distinct point $p_{A} \in A \backslash B$ whose ambient simplex $\Delta\left(p_{A}\right)$ is crossed by $H$, for a total of at least $\epsilon_{\delta} n$ of such points. Hence, $K$ must be $\epsilon_{\delta}$-narrow in $\mathcal{P}(s)$ and, thereby, already removed from $\mathcal{K}_{\text {surr }}$.

Back to the proof of Theorem 5.1. By definition, for every $K \in \mathcal{K}_{\text {surr }}$, the family $\left(\begin{array}{c}P_{K} \\ d+1\end{array}\right)$ contains a sub-collection $\Psi_{K}$ of at least $\delta\left(\begin{array}{c}\left|P_{K}\right| \\ d\end{array}\right)(d+1)$-subsets $A$ that are not vertically convex. By the previous Claim 5.2 at most $(\delta / 4)\left(\begin{array}{c}\left|P_{K}\right| \\ d+1\end{array}\right)$ of these $(d+1)$-subsets in $\Psi_{K}$ are degenerate.

Denote

$$
\mathcal{P}^{\prime}(s):=\left\{P_{i}|1 \leq i \leq s,| P_{i} \cap P_{K}\left|\leq \frac{\delta}{4 s(d+1)}\right| P_{K} \mid\right\}
$$

Since the sets of $\mathcal{P}^{\prime}(s)$ encompass at total of at most $\delta\left|P_{K}\right| /(4 d+4)$ points of $P_{K}$, at most $(\delta / 4)\left(\begin{array}{l}\left|P_{K}\right| \\ d+1\end{array}\right)$ of the $(d+1)$-sets $A \in \Psi_{K}$ can intersect $P_{K} \cap\left(\bigcup \mathcal{P}^{\prime}(s)\right)$.

In particular, there remains at least one regular $(d+1)$-set $A=\left\{p_{1}, \ldots, d+1\right\}$, in $\Psi_{K}$, so that $A \subset \bigcup\left(\mathcal{P}(s) \backslash \mathcal{P}^{\prime}(s)\right){ }^{24}$ Assume with no loss of generality that $p_{d+1}^{\perp} \in \operatorname{conv}\left(p_{1}^{\perp}, \ldots, p_{d}^{\perp}\right)$. By the regularity of $A$, we have that $\Delta\left(p_{d+1}\right)$ is surrounded by the simplices $\Delta\left(p_{i}\right)$, for $1 \leq i \leq d$. Furthermore, as $A$ is contained in $\bigcup \mathcal{P}(s) \backslash \mathcal{P}^{\prime}(s)$ (and it is not degenerate), the respective $d+1$ subsets $P_{j_{i}} \ni p_{i}$ of $\mathcal{P}(s)$ are distinct and satisfy $\left|P_{j_{i}}\right| \geq \delta\left|P_{K}\right| /((4 d+4) s)$ for all $1 \leq i \leq d+1$. Hence, $\left(P_{j_{1}}, \ldots, P_{j_{d+1}}\right)$ is a characteristic $(d+1)$-tuple, which completes the proof of Theorem 5.1 .

For every convex set we fix a unique characteristic $(d+1)$-tuple $\mathcal{T}(K)=\left(P_{j_{1}}, \ldots, P_{j_{d+1}}\right)$ as provided by Theorem 5.1 .

\subsection{The main simplicial partition in $\mathbb{R}^{d}$}

In this section we describe the main $d$-dimensional partition $\mathcal{Q}(s, t)$ of $P$ which will facilitate the final incidence-based argument of Sections 5.3, 5.4, and 5.5 (along the general lines sketched in Section 2.2. In process, we get rid of the convex sets $K \in \mathcal{K}_{\text {surr }}$ whose points are not sufficiently well-distributed amongst the parts of $\mathcal{Q}(s, t)$, or these points are not in a "sufficiently convex" position in $\mathbb{R}^{d}$ (so the Strong Epsilon-Net Theorem 2.3 can be invoked). To that end, we include in $N_{\text {surr }}$ several more elementary nets.

\footnotetext{
${ }^{24}$ With some abuse of notation, $\mathcal{P}(s)$ is treated as the collection $\left\{P_{1}, \ldots, P_{s}\right\}$ of its parts.
} 
The subsequent analysis builds on, and refines, the vertical partition $\mathcal{P}(s)$ that was introduced in the previous Section 5.1. We also use that every remaining convex set $K \in \mathcal{K}_{\text {surr }}$ is assigned a unique characteristic $(d+1)$-tuple $\mathcal{T}(K)=\left(P_{i_{1}}, \ldots, P_{i_{d+1}}\right)$ whose properties are detailed in the same Section 5.1 in the terms of the underlying vertical partition $\mathcal{P}(s)$. For the sake of brevity, in what follows we typically assume that $i_{j}=j$ for all $1 \leq j \leq d+1$, so $\mathcal{T}(K)=\left(P_{1}, \ldots, P_{d+1}\right)$.

The partition $\mathcal{Q}(s, t)$. We fix a parameter $t$ which is proportional to $1 / \epsilon^{c}$, for some positive (and substantial) constant $d / 2<c<d-1 / 2$ to be determined in Section 5.6. In particular, we have that $\frac{1}{s \epsilon^{d-1 / 2}} \gg t \ggg s \ggg 1 / \delta$, and assume that $n \geq 1 / \epsilon^{d-1 / 2}$. We construct the $d$-dimensional simplicial $t$-partition $\mathcal{Q}_{i}(t)=\left\{\left(Q_{i, j}, \varphi_{i, j}\right) \mid 1 \leq j \leq t\right\}$ of Theorem 2.2 for each set $P_{i}$ of the vertical $s$-partition $\mathcal{P}(s)$, and use $\mathcal{Q}(s, t)$ to denote the resulting partition $\biguplus_{i=1}^{s} \mathcal{Q}_{i}(t)$ of the entire point set $P$. (By the definition of $\mathcal{Q}(s, t)$, and the choice of $t$, none of the parts $Q_{i, j} \in \mathcal{Q}(s, t)$ is empty.)

In what follows, it will be instrumental to assume that the points of $P_{K}$, for $K \in \mathcal{K}_{\text {surr }}$, are reasonably well distributed amongst the parts $Q_{i, j}$ of $\mathcal{Q}(s, t)$. To that end, for the rest of this section we fix three parameters

$$
\epsilon^{*}:=C^{*} \frac{\delta \epsilon}{s t^{1-2 /(2 d-1)}}, \hat{\epsilon}:=\hat{C} \frac{\delta \epsilon}{s t^{1-1 / d}} \text { and } \tilde{\epsilon}_{n a r}:=\tilde{C}_{n a r} \epsilon \delta
$$

where $C^{*}>0, \hat{C}>0$, and $\tilde{C}_{n a r}>0$ are constants to be determined in the sequel.

Definition. We say that a convex set $K \in \mathcal{K}_{\text {surr }}$ is $\epsilon^{*}$-crowded in a part $Q_{i, j} \subseteq P_{i}$ of $\mathcal{Q}(s, t)$, for some $1 \leq i \leq s$ and $1 \leq j \leq t$, if it satisfies $\left|Q_{i, j} \cap P_{K}\right| \geq \epsilon^{*} n$. We say that $K \in \mathcal{K}_{\text {surr }}$ is $\epsilon^{*}$-crowded (in the partition $\mathcal{Q}(s, t)$ of the entire set $P$ ) if it is $\epsilon^{*}$-crowded in at least one part $Q_{i, j}$ of $\mathcal{Q}(s, t)$.

To discard the easy instances $K \in \mathcal{K}_{\text {surr }}$ (e.g., where the points of $P_{K}$ are $\epsilon^{*}$-crowded in any part $Q_{i, j}$ of $\mathcal{Q}(s, t)$, or they are not in a sufficiently convex position), we use elementary recurrence in $\epsilon$ to construct three auxiliary nets $N_{\text {crowd }}\left(\epsilon^{*}\right), N^{\triangle}(\hat{\epsilon})$, and $N^{\cup}\left(\tilde{\epsilon}_{\text {nar }}, u\right)$. To that end, we fix an additional integer parameter $u$ so that $s \lll u \lll t=o\left(1 / \epsilon^{d}\right)$. Similar to $s$ (and unlike $t$ ), the new parameter $u$ will be proportional to an arbitrary small albeit fixed, degree of $1 / \epsilon$.

The net $N_{\text {crowd }}\left(\epsilon^{*}\right)$. As the cardinality of each set $Q_{i, j} \in \mathcal{Q}(s, t)$ is $\Theta(n /(s t))$, to pierce the $\epsilon^{*}$-crowded set $K \in \mathcal{K}$ we can fix a suitable value

$$
\epsilon^{\prime}=\Theta\left(\frac{\epsilon^{*} n}{n /(s t)}\right)=\Theta\left(t^{2 /(2 d-1)} \delta \epsilon\right)
$$

and construct an auxiliary $\epsilon^{\prime}$-net $N_{i, j}$ for the family $\mathcal{K}\left(Q_{i, j}, \epsilon^{\prime}\right)$ for all $1 \leq i \leq s$ and $1 \leq j \leq s$.

Notice that the overall cardinality of the resulting net

$$
N_{\text {crowd }}\left(\epsilon^{*}\right):=\bigcup\left\{N_{i, j} \mid 1 \leq i \leq s, 1 \leq j \leq t\right\}
$$

is given by

$$
\left|N_{\text {crowd }}\left(\epsilon^{*}\right)\right|=O\left(s \cdot t \cdot f\left(\epsilon \delta \cdot t^{2 /(2 d-1)}\right)\right) .
$$

The strong net $N^{\triangle}(\hat{\epsilon})$. We invoke Theorem 2.3 to construct a strong $\hat{\epsilon}$-net $N^{\triangle}(\hat{\epsilon})$ that pierces every convex $(2 d)$-hedron that is $\hat{\epsilon}$-heavy with respect to $P$. Notice that 


$$
\left|N^{\triangle}(\hat{\epsilon})\right|=O\left(\frac{1}{\hat{\epsilon}} \log \frac{1}{\hat{\epsilon}}\right)=O\left(\frac{s t^{1-1 / d}}{\delta \epsilon} \log \frac{s t}{\delta \epsilon}\right) .
$$

The secondary vertical partitions $\mathcal{P}_{i}(u)$, and the net $N^{\cup}\left(\tilde{\epsilon}_{n a r}, u\right)$. In the subsequent steps, we routinely apply the Multiple Selection Theorem 3.3 in order to cross many of the simplices that are determined by the subsets $P_{i} \cap P_{K}$, for $P_{i} \in \mathcal{P}(s)$. Recall, though, that Theorem 3.3 too is formulated in the terms of a certain vertical simplicial partition of the underlying point set (in this case, $P_{i}$ ), and effectively requires that our set $K$ is sufficiently spread there.

Therefore, for each $1 \leq i \leq s$ we construct a secondary vertical simplicial $u$-partition $\mathcal{P}_{i}(u)$ of each set $P_{i} \in \mathcal{P}(s)$ (as described in Section 3.1). We then invoke Lemma 3.1 so as to pierce every convex set in $\mathcal{K}_{\text {surr }}$ that is $\tilde{\epsilon}_{\text {nar }}$-narrow with respect to the secondary partition $\mathcal{P}_{i}(u)$ of each part $P_{i}$ of $\mathcal{P}(s)$. This yields a net $N_{i}\left(\tilde{\epsilon}_{n a r}, u\right)$ of cardinality $O\left(u \cdot f\left(\tilde{\epsilon}_{n a r} \cdot u^{1 /(d-1)}\right)\right)=O\left(u \cdot f\left(\delta \epsilon u^{1 /(d-1)}\right)\right)$ for each $1 \leq i \leq s$.

We then define $N^{\cup}\left(\tilde{\epsilon}_{n a r}, u\right):=\bigcup_{i=1}^{s} N_{i}\left(\tilde{\epsilon}_{n a r}, u\right)$, so that

$$
\left|N^{\cup}\left(\tilde{\epsilon}_{n a r}, u\right)\right|=O\left(s u \cdot f\left(\delta \epsilon u^{1 /(d-1)}\right)\right) .
$$

We immediately add $N_{\text {crowd }}\left(\epsilon^{*}\right) \cup N^{\nabla}(\hat{\epsilon}) \cup N^{\cup}\left(\tilde{\epsilon}_{\text {nar }}, u\right)$ to our net $N_{\text {surr }}$, and remove from $\mathcal{K}_{\text {surr }}$ every convex set that is already pierced by these nets. Hence, in what follows, we can assume that no remaining set $K \in \mathcal{K}_{\text {surr }}$ is $\epsilon^{*}$-crowded in any part of our partition $\mathcal{Q}(s, t)$, any convex $(2 d)$-hedron within $K \in \mathcal{K}_{\text {surr }}$ contains at most $\hat{\epsilon} n$ points of $P$, and each convex set $K \in \mathcal{K}_{\text {surr }}$ is $\tilde{\epsilon}_{n a r}$-spread in the secondary vertical $u$-partition $\mathcal{P}_{i}(u)$ of any set $P_{i} \in \mathcal{P}(s)$, for $1 \leq i \leq s$.

The net $N_{\text {sparse }}$. Let $K \in \mathcal{K}_{\text {surr }}$ be a convex set with the characteristic $(d+1)$-tuple $\mathcal{T}(\mathcal{K})$. (To simplify our notation, we assumed with no loss of generality that $\mathcal{T}(K)=\left(P_{1}, \ldots, P_{d+1}\right)$.) Then the ambient simplex $\Delta_{d+1}$ of the last set $P_{d+1}$ is surrounded by the ambient simplices $\Delta_{i} \supset P_{i}^{\perp}$ of the remaining $d$ sets $P_{i}$, for $1 \leq i \leq d$. By definition, we have that $\left|P_{i} \cap P_{K}\right| \geq C_{\text {surr }} \cdot \delta\left|P_{K}\right| / s$.

To facilitate the subsequent steps, it will instrumental to assume that $P_{K} \cap P_{d+1}$ contains a large subset $Q_{K}$ whose points belong to the called full parts $Q_{d+1, j}$ in the decomposition $\mathcal{Q}_{d+1}(t)$ of $Q_{d+1}$; each full part $Q_{d+1, j} \in \mathcal{Q}_{d+1}(t)$ will encompass $\Omega\left(\delta\left|P_{K}\right| /\left(s t^{1-1 / d}\right)\right)=\Omega(\hat{\epsilon} n)$ points of $P_{K} \cap P_{d+1}$.

To dispatch the remaining convex sets $K \in \mathcal{K}_{\text {surr }}$, whose respective subsets $P_{K} \cap P_{d+1}$ are somewhat more sparsely distributed amongst $\Omega\left(t^{1-1 / d}\right)$ parts of $\mathcal{Q}_{d+1}(t)$, we will introduce an additional net $N_{\text {sparse }}$. To this end, for each $1 \leq i \leq s$ we apply our Multiple Selection Theorem 3.3 with respect to the underlying set $P_{i}$, and its secondary $(d-1)$-dimensional partition $\mathcal{P}_{i}(u)$; this yields a set $\mathcal{L}_{i}(u)$ of at most $u^{(d-1)^{2}}$ vertical lines which depends only on the secondarly partition $\mathcal{P}_{i}(u)$ of $P_{i}$. For each point $p \in P_{i}$, we clip the ambient simplex $\varphi(p)$ of $p$ in the simplicial partition $\mathcal{Q}_{i}(t)$ of $P_{i}$, to the vertical prism above the ambient simplex of $p$ in $\mathcal{P}_{i}(u)$, and refer to the resulting polytope as the clipped simplex $\tilde{\varphi}(p)$.

For each $1 \leq i \leq s$, we denote $\Phi_{i}:=\left\{\tilde{\varphi}(p) \mid p \in P_{i}\right\}$. (Note that all the points $p \in P_{i}$ that have the same ambient simplices in $\mathcal{P}_{i}(u)$ and $\mathcal{Q}_{i}(t)$ are assigned to the same clipped simplex $\tilde{\varphi}(p) \in \Phi_{i}$.) Clearly, we have that $\left|\Phi_{i}\right| \leq u t$, and each of its clipped simplices is a convex polyhedron with at most $2 d+1$ facets. Let $V_{i}$ denote the set of all the vertices of the clipped simplices of $\Phi_{i}$.

For each line $\ell \in \mathcal{L}_{i}(u)$ let $X_{\ell}$ denote the set of all the $\ell$-intercepts $\ell \cap \operatorname{conv}(\tau)$ of the $\operatorname{simplices}$ $\tau \in\left(\begin{array}{c}V_{i} \\ d\end{array}\right)$; see Figure 15 . We add every $\left\lceil C_{\text {sparse }} t^{d-1}\right\rceil$-th point of $X_{\ell}$ to the local net $N_{\ell}$, and denote $N_{\text {sparse }}:=\bigcup_{i=1}^{S} \bigcup_{\ell \in \mathcal{L}_{i}(u)} N_{\ell}$. 


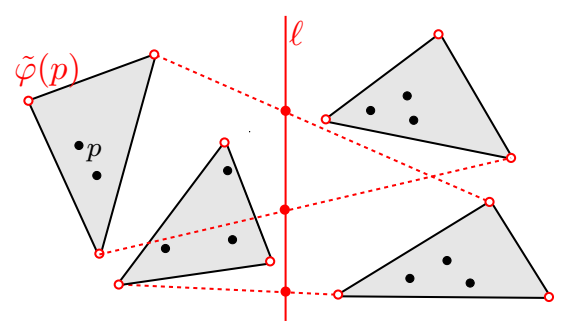

Figure 15: The net $N_{\text {sparse }}$ - a schematic description (in dimension $d=2$ ). For each $1 \leq i \leq s$, the collection $\Phi_{i}$ is comprised of all the (clipped) simplices $\tilde{\varphi}(p)$, for $p \in P_{i}$, and $V_{i}$ consists of all the vertices of $\Phi_{i}$. For each $\ell \in \mathcal{L}_{i}(u)$, the net $N_{\ell}$ is constructed over the set $X_{\ell} \subseteq \ell$ of all the $\ell$-intercepts of the simplices of $\left(\begin{array}{c}V_{i} \\ d\end{array}\right)$.

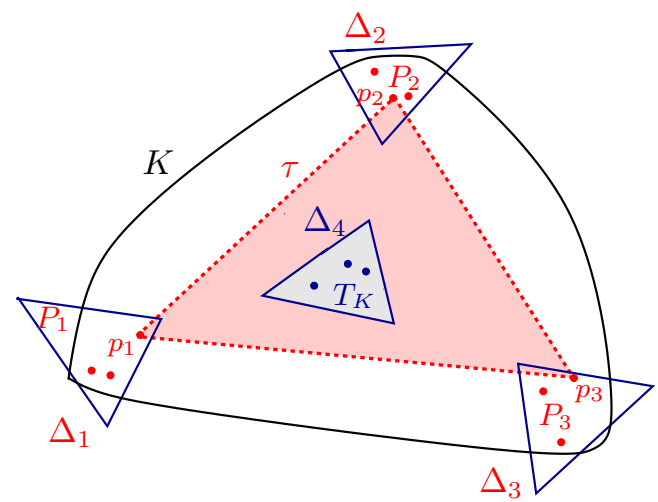

Figure 16: The sets $T_{K}, S_{K}$, and $\Sigma_{K}$ - view from above in dimension $d=3$. The set $T_{K}$ (blue) includes $\left\lceil C_{\text {surr }} \cdot \delta \epsilon n / s\right\rceil$ points from $P_{K} \cap P_{4}$, and the set $S_{K}$ (red) includes $\left\lceil C_{\text {surr }} \cdot \delta \epsilon n / s\right\rceil$ points from each subset $P_{K} \cap P_{i}$, for $1 \leq i \leq 3$. The set $\Sigma_{K}$ is comprised of all the simplices $\tau=\operatorname{conv}\left(p_{1}, p_{2}, p_{3}\right)$, for $p_{i} \in S_{K} \cap P_{i}$. Since $\Delta_{4}$ is surrounded, the projection of each simplex $\tau \in \Sigma_{K}$ contains $\Delta_{K}$ and $T_{K}^{\perp}$.

Since $\left|X_{\ell}\right|=O\left(\left(\begin{array}{c}u t \\ d\end{array}\right)\right)$ for all $\ell \in \mathcal{L}_{i}(u)$, and all $1 \leq i \leq s$, we have that

$$
\left|N_{\text {sparse }}\right|=O\left(s u^{(d-1)^{2}+d} \cdot t\right)
$$

which completes the description of $N_{\text {sparse }}$. We add the points of $N_{\text {sparse }}$ to $N_{\text {surr }}$ and, accordingly, remove from $\mathcal{K}_{\text {surr }}$ every convex set that is pierced by $N_{\text {sparse }}$.

The properties of $N_{\text {sparse }}$. It, therefore, remains to check that $N_{\text {sparse }}$ satisfies the asserted properties with a suitable choice of the frequency constant $C_{\text {sparse }}>0$. To this end, we consider a convex set $K \in \mathcal{K}_{\text {surr }}$, with the characteristic $(d+1)$-tuple $\mathcal{T}(K)=\left(P_{1}, \ldots, P_{d+1}\right)$. We fix a set $T_{K} \subset P_{K} \cap P_{d+1}$ of cardinality $\left\lceil C_{\text {surr }} \cdot \delta \epsilon n / s\right\rceil$, and another set $S_{K} \subseteq P_{K} \cap\left(\bigcup_{i=1}^{d} P_{i}\right)$ which includes $\left\lceil C_{\text {surr }} \cdot \delta \epsilon n / s\right\rceil$ points from each intersection $P_{i} \cap P_{K}$, for $1 \leq i \leq d$. We then denote

$$
\Sigma_{K}:=\left\{\operatorname{conv}\left(p_{1}, p_{2}, \ldots, p_{d}\right) \mid p_{1} \in S_{K} \cap P_{1}, p_{2} \in S_{K} \cap P_{2}, \ldots, p_{d} \in S_{K} \cap P_{d}\right\} .
$$

Since $\Delta_{d+1} \supset P_{d+1}^{\perp} \supset\left(T_{K}\right)^{\perp}$ is surrounded by the remaining $d$ simplices $\Delta_{i} \supset P_{i}^{\perp}$, for $1 \leq i \leq d$, the projection $p^{\perp}$ of every point $p \in T_{K}$ falls within the respective projection $(\operatorname{conv}(\tau))^{\perp}$ of every simplex $\tau \in \Sigma_{K}$. See Figure 16 . 


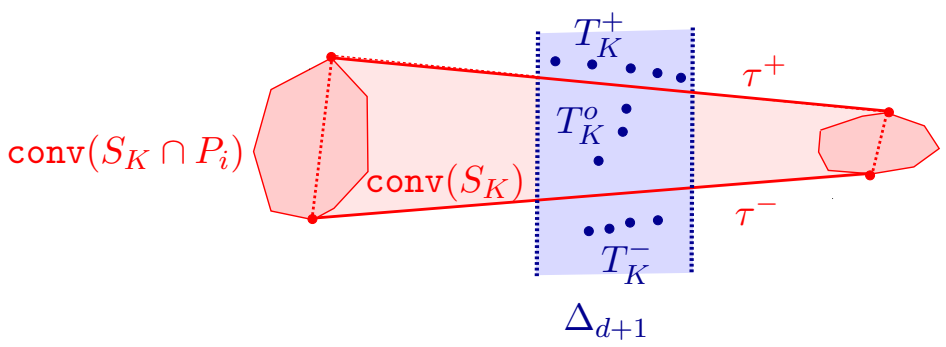

Figure 17: Proof of Proposition 5.3 - a schematic summary. The points of $T_{K}^{o}$ lie in the prism above $\Delta_{d+1}$, and are sandwiched in the $(2 d)$-hedron $M=\operatorname{conv}\left(\tau^{-} \cup \tau^{+}\right)$, where $\tau^{+}$(resp., $\tau^{-}$) is topmost (lowernost) facet of $\operatorname{conv}\left(S_{K}\right)$.

Definition. We say that a point $p \in T_{K}$ lies above (resp., below) a simplex $\tau \in \Sigma_{K}$ if the vertical line through $p$ meets $\tau$ below $p$ (resp., above $p$ ). Let $T_{K}^{+} \subseteq T_{K}$ (resp., $T_{K}^{-} \subseteq T_{K}$ ) denote the subset of all the points in $T_{K}$ that lie above (resp., below) every simplex of $\Sigma_{K}$, and denote $T_{K}^{o}:=T_{K} \backslash\left(T_{K}^{+} \cup T_{K}^{-}\right)$.

Proposition 5.3. The set $T_{K}^{o}$ has cardinality at most $\hat{\epsilon} n$.

Proof. Since $\Delta_{d+1}$ is surrounded in $\mathbb{R}^{d-1}$ by $\Delta_{1}, \ldots, \Delta_{d}$, the projection $\left(T_{K}^{o}\right)^{\perp} \subset \Delta_{d+1}$ is contained in the projection of every simplex of $\Sigma_{K}$. The vertical line $\ell_{p}$ through any point $p \in T_{K}^{o}$ meets the boundary of conv $\left(S_{K}\right)$ at the simplices $\tau^{-} \in \Sigma_{K}$ and $\tau^{+} \in \Sigma_{K}$, in this ascending order of the last coordinate. Furthermore, it readily follows that $\tau^{-}$and $\tau^{+}$are the only two facets of $\operatorname{conv}\left(S_{K}\right)$ that belong to $\Sigma_{K}{ }^{25}$ In particular, $\tau^{-}$and $\tau^{+}$do not depend on the choice of $p \in T_{K}^{o}$. Consider the convex $2 d$-hedron $M:=\operatorname{conv}\left(\tau^{-} \cup \tau^{+}\right)$; see Figure 17 . The claim now follows since $T_{K} \cap M=T_{K}^{o}$, and $|M \cap P| \leq \hat{\epsilon} n$.

Notice that the sets $T_{K}, S_{K}, T_{K}^{+}, T_{K}^{-}$and $\Sigma_{K}$ are uniquely determined in the above manner for each convex set $K \in \mathcal{K}_{\text {surr }}$ (through the combination of $P_{K}$ and the characteristic $(d+1)$-tuple $\mathcal{T}(K))$.

Definition. Let $\mathcal{K}^{+} \subseteq \mathcal{K}_{\text {surr }}$ denote the subfamily of all the convex sets $K \in \mathcal{K}_{\text {surr }}$ so that $\left|T_{K}^{+}\right| \geq\left|T_{K}^{-}\right|$. By Proposition 5.3 , we have that $\left|T_{K}^{+}\right| \geq\left|T_{K}\right| / 3 \geq C_{\text {surr }} \cdot \delta \epsilon n /(3 s)$ for every $K \in \mathcal{K}^{+}$. We denote $\mathcal{K}^{-}:=\mathcal{K}_{\text {surr }} \backslash \mathcal{K}^{+}$, and observe that its convex sets satisfy the symmetric inequality $\left|T_{K}^{-}\right| \geq\left|T_{K}\right| / 3$. With no loss of generality, our analysis in the sequel concerns only the sets of $\mathcal{K}^{+}$; the complementary family $\mathcal{K}^{-}$can be dispatched in a fully symmetrical manner.

To further simplify our notation, we refer to the parts of the simplicial partition $\mathcal{Q}_{d+1}(t)$ as $Q_{1}, \ldots, Q_{t}$. Let us denote $Q_{K, j}:=T_{K}^{+} \cap Q_{j}$ for each $K \in \mathcal{K}^{+}$and $Q_{j} \in Q_{d+1}(t)$.

Definition. We say that the part $Q_{j} \in \mathcal{Q}_{d+1}(t)$, for $1 \leq j \leq t$, is full with respect to $K$ if it satisfies

$$
\left|Q_{K, j}\right| \geq C_{\text {full }} \cdot \frac{\delta \epsilon n}{s t^{1-1 / d}},
$$

\footnotetext{
${ }^{25}$ Note that $\tau^{-}$(resp., $\tau^{+}$) supports the unique lower (resp., upper) common tangent hyperplane $H\left(\tau^{-}\right)$(resp., $\left.H\left(\tau^{+}\right)\right)$to the sets $d$ sets $\operatorname{conv}\left(S_{K} \cap P_{i}\right)$, for $1 \leq i \leq s$.
} 
where the constant $C_{\mathrm{full}}>0$ will be chosen so as to suit Proposition 5.4 below.

Definition. Let $\mathcal{Q}_{K}$ denote the subset of all the full parts in $\mathcal{Q}_{d+1}(t)$ with respect to $K$. By definition, we have that $\left|\mathcal{Q}_{K}\right|=O\left(t^{1-1 / d}\right)$.

Denote $Q_{K}:=T_{K}^{+} \cap\left(\bigcup \mathcal{Q}_{K}\right)=\bigcup_{j \in[t]: Q_{j} \in \mathcal{Q}_{K}} Q_{K, j}$. In what follows, we refer to $Q_{K}$ as the characteristic subset of $K$.

Similar to $P_{K}, S_{K}, T_{K}$, and $\Sigma_{K}$, the sets $\mathcal{Q}_{K}$ and $Q_{K}$ are fully determined by the choice of $K \in \mathcal{K}^{+}$.

Proposition 5.4. For any small enough constants $\hat{C}>0, \tilde{C}_{n a r}>0, C_{\text {sparse }}>0$, and $C_{\text {full }}>0$, every remaining convex set $K \in \mathcal{K}^{+}$satisfies

$$
\left|Q_{K}\right| \geq\left|T_{K}^{+}\right| / 2 \geq\left\lceil C_{\text {surr }} \cdot \delta \epsilon n /(6 s)\right\rceil .
$$

Proof. We establish the proposition with $C_{\text {full }}=1 /\left(10 c_{d}\right)$, where $c_{d}>0$ is the constant in the Simplicial Partition Theorem 2.2. Fix a convex set $K \in \mathcal{K}^{+}$. Suppose that $Q_{K}$ does not satisfy the asserted inequality, so at least half of the points of $T_{K}^{+}$belong to the parts of $\mathcal{Q}_{d+1}(t) \backslash \mathcal{Q}_{K}$. It is sufficient to show that, given a suitably small choice of the constant $\hat{C}, \tilde{C}_{n a r}$, and $C_{\text {sparse }}$, the set $K$ is pierced by the net $N_{\text {sparse }}$, which is contrary to its inclusion in $\mathcal{K}^{+}$.

Fix a simplex $\tau \in \Sigma_{K}$. Then its supporting hyperplane $H(\tau)$ crosses (the ambient simplices of) at most $c_{d} t^{1-1 / d}$ parts $\mathcal{Q}_{d+1}(t) \backslash \mathcal{Q}_{K}$. Therefore, and since any part $Q_{j} \in \mathcal{Q}_{d+1}(t) \backslash \mathcal{Q}_{K}$ contains fewer than $\left|T_{K}^{+}\right| /\left(10 c_{d} t^{1-1 / d}\right)$ points of $T_{K}^{+}$(by the definition of a full part $Q_{j}$ ), the zone of $H(\tau)$ in $\mathcal{Q}_{d+1}(t)$ accounts for at most $\left|T_{K}^{+}\right| / 10$ points of $T_{K}^{+} \backslash Q_{K}$.

Let $W_{K}$ denote the subset of all the points in $T_{K}^{+} \backslash Q_{K}$ that lie outside the zone of $H(\tau)$. The preceding discussion implies that $\left|W_{K}\right| \geq 9\left|T_{K}^{+}\right| / 10=\Omega(\delta \epsilon n / s)=\Omega\left(\delta \epsilon\left|P_{d+1}\right|\right)$. Notice that for every point $p \in W_{K}$, both its ambient simplex $\varphi(p)$ in $\mathcal{Q}(s, t)$, and its finer clipped counterpart $\tilde{\varphi}(p) \in \Phi_{d+1}$, lie entirely above $H(\tau)$.

Since $K$ is $\tilde{\epsilon}_{n a r}$-spread (for $\tilde{\epsilon}_{n a r}=\tilde{C}_{n a r} \delta \epsilon$ ) in $\mathcal{P}_{d+1}(u)$, and given any suitably small choice of the constant $\tilde{C}_{n a r}>0$, Theorem 3.3 yields a line $\ell \in \mathcal{L}_{d+1}(u)$ that pierces a subset $\Xi_{K}(\ell) \subseteq\left(\begin{array}{c}W_{K} \\ d\end{array}\right)$ of cardinality $\Omega\left(\left(\begin{array}{c}\left|W_{K}\right| \\ d\end{array}\right)\right)$. Fix a $(d-1)$-simplex $\mu=\operatorname{conv}\left(q_{1}, \ldots, q_{d}\right) \in \Xi_{K}(\ell)$. Notice that $\ell$ is surrounded by the respective ambient simplices $\tilde{\Delta}\left(q_{1}\right), \ldots, \tilde{\Delta}\left(q_{d}\right)$ of the points $q_{1}, \ldots, q_{d}$ in the vertical partition $\mathcal{P}_{d+1}(u)$. For each $1 \leq i \leq d$ we pick a vertex $v_{i}$ of the ambient clipped simplex $\tilde{\varphi}\left(q_{i}\right)$, that lies above $H(\tau)$ yet below the supporting hyperplane plane $H(\mu)$ of $\mu$. Such a vertex $v_{i}$ exists because (i) $H(\mu)$ crosses the interior of every clipped simplex $\tilde{\varphi}\left(q_{i}\right)$, for $1 \leq i \leq d$, at the respective point $q_{i}$, and (ii) each $\tilde{\varphi}\left(q_{i}\right)$ lies entirely above $H(\tau)$. Notice that we have $v_{i} \in V_{d+1}$ for all $1 \leq i \leq d$. See Figure 18 .

We assign $\mu$ to the resulting simplex $\tilde{\mu}=\operatorname{conv}\left(v_{1}, \ldots, v_{d}\right) \in\left(\begin{array}{c}V_{d+1} \\ d\end{array}\right)$. Note that $\tilde{\mu}$ too is crossed by the line $\ell$, for the projections $v_{1}^{\perp}, \ldots, v_{d}^{\perp}$ lie in the simplices $\tilde{\Delta}\left(q_{1}\right), \ldots, \tilde{\Delta}\left(q_{d}\right)$.

We claim that the three intercepts $\tau \cap \ell, \tilde{\mu} \cap \ell$, and $\mu \cap \ell$ appear in this ascending order along $\ell$; in particular, the interval $K \cap \ell$ contains the point $\tilde{\mu} \cap \ell$. To see that $\ell \cap \tilde{\mu}$ is above $\ell \cap \tau$, recall that all the $d$ vertices $v_{1}, \ldots, v_{d}$ lie above $H(\tau)$ so (by convexity) the point $\ell \cap \tilde{\mu} \in \operatorname{conv}\left(v_{1}, \ldots, v_{d}\right)$ too lies above $H(\tau)$. A similar claim argument shows that $\ell \cap \tilde{\mu}$ is below $\ell \cap \mu$ (using that all the $d$ vertices of $\tilde{\mu}$ lie below $H(\mu))$.

Since $O\left(\left|W_{K}\right| / t^{1-1 / d}\right)$ points of $W_{K}$ lie in any single part of $\mathcal{Q}_{d+1}(t)$ (for $\left.W_{K} \cap\left(\bigcup \mathcal{Q}_{K}\right)=\emptyset\right)$, any simplex $\tilde{\mu} \in\left(\begin{array}{c}V_{d+1} \\ d\end{array}\right)$ is assigned

$$
O\left(\frac{\left|W_{K}\right|}{t^{1-1 / d}}\right)^{d}
$$




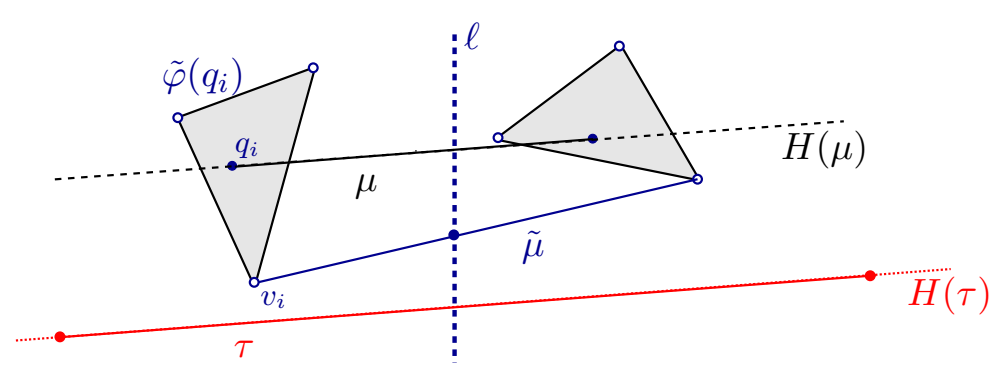

Figure 18: Proof of Proposition 4.6 Assigning a simplex $\mu=\operatorname{conv}\left(q_{1}, \ldots, q_{d}\right) \in\left(\begin{array}{c}W_{K} \\ d\end{array}\right)$ to a simplex $\tilde{\mu} \in V_{d+1}$. For each $q_{i}$, its ambient clipped simplex $\tilde{\varphi}\left(q_{i}\right)$ has a vertex that lies above $H(\tau)$ yet below $H(\mu)$. Since $\ell$ is surrounded, the line $\ell \in \mathcal{L}_{d+1}(u)$ meets the simplex $\tilde{\mu}=\operatorname{conv}\left(v_{1}, \ldots, v_{d}\right)$ within $K$, between $\mu$ and $\tau$.

simplices $\mu \in \Xi_{K}(\ell)$. Therefore, the interval $\ell \cap K$ contains $\Omega\left(t^{d-1}\right)$ intercepts $\ell \cap \tilde{\mu}$ of the simplices $\tilde{\mu} \in\left(\begin{array}{c}V_{d+1} \\ d\end{array}\right)$. Hence, given a suitably small constant $C_{\text {sparse }}>0$, the interval $K \cap \ell$ must contain a point of $N_{\ell} \subset N_{\text {sparse }}$.

In what follows, we assume that the constants $\hat{C}>0, C_{\text {full }}>0, \tilde{C}_{\text {nar }}>0$, and $C_{\text {sparse }}>0$ suit the criteria of Proposition 5.4. As a result, every remaining convex set $K \in \mathcal{K}_{\text {surr }}$ can be assigned the unique characteristic set $Q_{K} \subseteq T_{K}^{+} \cap\left(\bigcup \mathcal{Q}_{K}\right)$ of cardinality $\left|Q_{K}\right| \geq C_{\text {surr }} \cdot \delta \epsilon n /(6 s)$.

\subsection{Further properties of the sets $K \in \mathcal{K}^{+}$}

Fix a convex set $K \in \mathcal{K}^{+}$. As described in the previous Section 5.2 , the set $K$ uniquely determines not only $P_{K}$ and the characteristic $(d+1)$-tuple $\mathcal{T}(K)=\left(P_{1}, \ldots, P_{d+1}\right)$, but also the subsets $T_{K} \subseteq P_{K} \cap P_{d+1}$, and $S_{K} \subset P_{K} \cap\left(\bigcup_{i=1}^{d} P_{i}\right)$, which determines the collection of $\Theta\left((\delta \epsilon n)^{d} / s^{d}\right)$ simplices $\Sigma_{K}:=\left\{\operatorname{conv}\left(p_{1}, \ldots, p_{d}\right) \mid p_{1} \in S_{K} \cap P_{1}, p_{2} \in S_{K} \cap P_{2}, \ldots, p_{d} \in S_{K} \cap P_{d}\right\}$.

In addition, $K$ determines the collection $\mathcal{Q}_{K} \in \mathcal{Q}_{d+1}(t)$ of the full parts whose union $\bigcup \mathcal{Q}_{K}$ cuts out the characteristic subset $Q_{K} \subseteq T_{K}$, of cardinality at least $\left\lceil C_{\text {surr }} \delta \epsilon n /(6 s)\right\rceil$, and so that every point of $Q_{K}$ lies above every simplex $\tau \in \Sigma_{K}$. In the sequel, we will use that each full part encompasses at least $\left\lceil C_{\text {full }} \cdot \delta \epsilon n /\left(s t^{1-1 / d}\right)\right\rceil$ points of $Q_{K}$.

The complete hypergraphs $\Lambda_{K, j}$. For each $Q_{j} \in \mathcal{Q}_{K}$ we construct the complete hypergraph over the set $Q_{K, j}=Q_{K} \cap Q_{j}$ :

$$
\Lambda_{K, j}:=\left(\begin{array}{c}
Q_{K, j} \\
d
\end{array}\right)
$$

Since the part $Q_{j} \in \mathcal{Q}_{K}$ is full, we have that

$$
\left|\Lambda_{K, j}\right| \geq\left(\begin{array}{c}
\left\lceil C_{\text {full }} \cdot \frac{\delta \epsilon n}{s t^{1-1 / d}}\right\rceil \\
d
\end{array}\right)=\Omega\left(\frac{(\delta \epsilon n)^{d}}{s^{d} t^{d-1}}\right) .
$$

We then denote

$$
\Lambda_{K}:=\bigcup_{j \in[t]: Q_{j} \in \mathcal{Q}_{K}} \Lambda_{K, j}
$$

Namely, $\Lambda_{K}$ is comprised of all the $(d-1)$-dimensional simplices in $\left(\begin{array}{c}Q_{K} \\ d\end{array}\right)$ so that all of their respective vertices come from the same part of $\mathcal{Q}_{d+1}(t)$. 

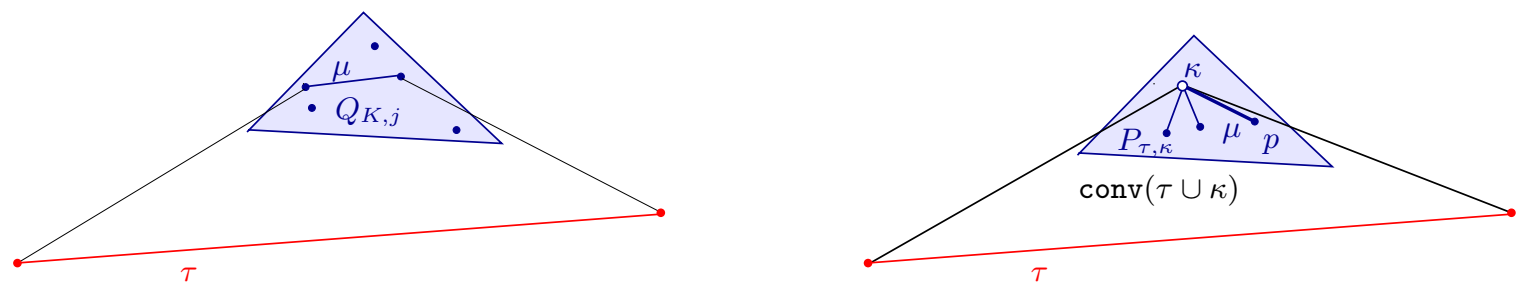

Figure 19: Left - The simplices $\tau \in \Sigma_{K}$ and $\mu \in \Lambda_{K, j}$ are parallel - they are facets of a convex (2d)-hedron $\operatorname{conv}(\tau \cup \mu)$. Right - Proof of Proposition 5.5. If $\tau \in \Sigma_{K}$ and $\mu \in \Lambda_{\tau}$ are not parallel. Then there is a $(d-2)$-face $\kappa \subset \mu$ so that the remaining vertex $p$ of $\mu$ lies in $\operatorname{conv}(\tau \cup \kappa)$, and is added to $P_{\tau, \kappa}$. The points of $P_{\mu, \kappa}$ are depicted.

Our small-size net for $\mathcal{K}^{+}$is based on the sensitive relation between the simplices of $\Sigma_{K}$ and $\Lambda_{K}$, for each $K \in \mathcal{K}^{+}$. Hence, the rest of this section is devoted to the interplay between these sets of simplices. In our analysis, we will use that no $K \in \mathcal{K}^{+}$is pierced by the previous nets $N^{\triangle}(\hat{\epsilon})$, $N^{\cup}\left(\epsilon, \tilde{\epsilon}_{\text {nar }}\right)$, and $N_{\text {crowd }}\left(\epsilon^{*}\right)$.

Definition. We say the simplices $\tau, \mu \in\left(\begin{array}{l}P \\ d\end{array}\right)$ are parallel if both $\tau$ and $\mu$ are facets of the convex $(2 d)$-hedron $\operatorname{conv}(\tau \cup \mu)$; see Figure 19 (left).

Fix $K \in \mathcal{K}^{+}$and recall that for any (not necessarily parallel) pair $(\tau, \mu) \in \Sigma_{K} \times \Lambda_{K}$, all the $d$ vertices of $\mu$ lie above $\tau$ (and, in particular, to the same side of $H(\tau)$ ). Hence, $\tau$ is always a facet of $\operatorname{conv}(\tau \cup \mu)$. Therefore, if $\tau$ and $\mu$ are not parallel, then at least one of the vertices $p \in Q_{K}$ of $\mu$ must lie in the convex hull of $\tau$ and the remaining $d-1$ vertices of $\mu$.

Proposition 5.5. Given a small enough constant $\hat{C}>0$ in the definition of the net $N^{\triangle}(\hat{\epsilon})$ (in Section 5.2), for each $K \in \mathcal{K}^{+}$, and each full part $Q_{j} \in \mathcal{Q}_{K}$, there exist at least $\left|\Sigma_{K}\right|\left|\Lambda_{K, j}\right| / 2$ parallel pairs in $\Sigma_{K} \times \Lambda_{K, j}$.

Proof. Assume for a contradiction that there exists a full part $Q_{j} \in \mathcal{Q}_{K}$ so that at least $\left|\Sigma_{K}\right|\left|\Lambda_{K, j}\right| / 2$ of the pairs $(\tau, \mu) \in \Sigma_{K} \times \Lambda_{K, j}$ are not parallel. For each of these pairs $(\tau, \mu)$, there a vertex $p$ of $\mu$ with the opposite a $(d-2)$-face $\kappa \subset \mu$ (not adjacent to $p$ ), so that $p$ lies in $\operatorname{conv}(\tau \cup \kappa)$. See Figure 19 (right). By the pigeonhole principle, there are $\tau \in \Sigma_{K}$, and a subset $\Lambda_{\tau}$ of at least $\left|\Lambda_{K, j}\right| / 2$ simplices $\mu \in \Lambda_{K}$ that are not parallel to $\tau$.

We label every simplex $\mu \in \Lambda_{\tau}$ with a $(d-2)$-face $\kappa \in\left(\begin{array}{c}Q_{K, j} \\ d-1\end{array}\right)$ of $\mu$ so that $\operatorname{conv}(\tau \cup \kappa)$ contains the remaining vertex of $\mu \sqrt{26}$ For each $\kappa \in\left(\begin{array}{c}Q_{K, j} \\ d-1\end{array}\right)$ let $P_{\tau, \kappa}$ denote the subset of all the points $p \in Q_{K, j}$ so that the simplex $\operatorname{conv}(\kappa \cup\{p\}) \in \Lambda_{\tau}$ is labelled with $\kappa$. The crucial observation is that $P_{\tau, \kappa}$ is entirely contained in the $(2 d-1)$-hedron $\operatorname{conv}(\tau \cup \kappa)$.

Another application of the pigeonhole principle yields $\kappa \in\left(\begin{array}{c}Q_{K, j} \\ d-1\end{array}\right)$ that satisfies

$$
\left|P_{\tau, \kappa}\right|=\Omega\left(\frac{\left|\Lambda_{\tau}\right|}{\left(\begin{array}{c}
\left|Q_{K, j}\right| \\
d-1
\end{array}\right)}\right)=\Omega\left(\frac{\left(\begin{array}{c}
\left|Q_{K, j}\right| \\
d
\end{array}\right)}{\left(\begin{array}{c}
\left|Q_{K, j}\right| \\
d-1
\end{array}\right)}\right)=\Omega\left(\frac{\delta \epsilon n}{s t^{1-1 / d}}\right) .
$$

That is, given a small enough choice of $\hat{C}>0$, there must be a convex $(2 d-1)$-hedron $\operatorname{conv}(\tau \cup \kappa)$ within $K$ that contains at least $\left|P_{\tau, \kappa}\right| \geq \hat{\epsilon} n$ points of $P$. The contradiction follows since such a convex set $K \in \mathcal{K}^{+}$must have been pierced by the strong net $N^{\triangle}(\hat{\epsilon})$.

\footnotetext{
${ }^{26}$ The may be several such choices of $\kappa$ if $\operatorname{conv}(\tau \cup \mu)$ has fewer than $2 d-1$ vertices.
} 

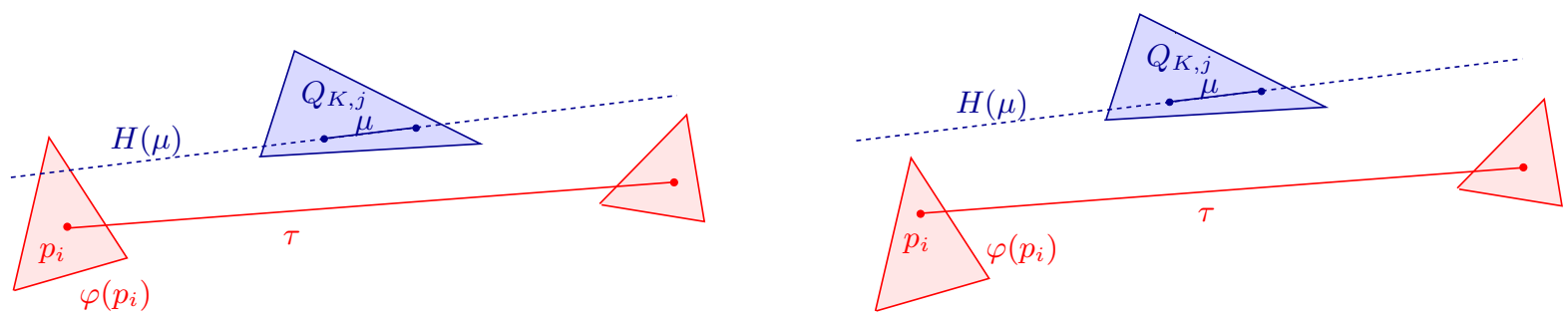

Figure 20: Left - The pair $(\tau, \mu) \in \Sigma_{K} \times \Lambda_{K}$ is tight: One of the vertices vertex $v_{i} \in S_{K} \cap P_{i}$ of $\tau$ lies in the zone of $H(\mu)$ within the partition $\mathcal{Q}_{i}(t)$ of $P_{i}$. That is, the ambient simplex $\varphi\left(p_{i}\right)$ of $p_{i}$ is crossed by $H(\mu)$. Right - The pair $(\tau, \mu) \in \Sigma_{K} \times \Lambda_{K}$ is loose: $H(\mu)$ misses all the ambient $\varphi\left(p_{i}\right)$ of the vertices $p_{i}$ of $\tau$.

Definition. We say that a pair of parallel simplices $(\tau, \mu) \in \Sigma_{K} \times \Lambda_{K}$ is tight if at least one of the vertices $p_{i} \in S_{K} \cap P_{i}$ of $\tau=\operatorname{conv}\left(p_{1}, \ldots, p_{d}\right)$ lies in the zone of $H(\mu)$ within the simplicial partition $\mathcal{Q}_{i}(t)$ of $P_{i}$ (so its ambient simplex $\varphi\left(p_{i}\right)$ in the partition $\mathcal{Q}_{i}(t)$ is crossed by the hyperplane $H(\mu)$ ). Otherwise, we say that the pair $(\tau, \mu)$ is loose. See Figure 20.

We say that a full part $Q_{j} \in \mathcal{Q}_{K}$ is tight (with respect to $K$ ) if at least $\left|\Lambda_{K, j}\right| \times\left|\Sigma_{K}\right| / 4$ of the pairs in $\Lambda_{K, j} \times \Sigma_{K}$ are tight, and we say that $Q_{j}$ is loose otherwise. In the latter case, Proposition 5.5 implies that at least $\left|\Lambda_{K, j}\right| \times\left|\Sigma_{K}\right| / 4$ of the pairs in $\Lambda_{K, j} \times \Sigma_{K}$ are loose.

Let $\mathcal{Q}_{K, \text { tight }} \subset \mathcal{Q}_{K}$ (resp., $\mathcal{Q}_{K \text {,loose }} \subset \mathcal{Q}_{K}$ ) denote the sub-collection of all the tight (resp., loose) parts in $\mathcal{Q}_{K}$. (Note that $\mathcal{Q}_{K}=\mathcal{Q}_{K, \text { tight }} \uplus \mathcal{Q}_{K, \text { loose }}$.)

Finally, we say that a convex set $K \in \mathcal{K}^{+}$is tight if $\left|Q_{K} \cap\left(\cup \mathcal{Q}_{K, \text { tight }}\right)\right| \geq\left|Q_{K}\right| / 2$, and otherwise we say that $K$ is loose (in which case we have $\left.\left|Q_{K} \cap\left(\bigcup \mathcal{Q}_{K, \text { loose }}\right)\right| \geq\left|Q_{K}\right| / 2\right)$.

Piercing the sets of $\mathcal{K}^{+}$- the roadmap. Let $\mathcal{K}_{\text {tight }}^{+}$(resp., $\mathcal{K}_{\text {loose }}^{+}$) denote the sub-family of all the tight (resp., loose) convex sets in $\mathcal{K}^{+}$.

In Section 5.4 we use Theorem 3.7 to construct a net $N_{\text {tight }}$ for the family $\mathcal{K}_{\text {tight }}$. To that end, we first define a relatively sparse subset of hyperedges $\Pi_{\text {rich }} \subseteq\left(\begin{array}{l}P \\ d\end{array}\right)\left(\right.$ of cardinality $\left|\Pi_{\text {rich }}\right|=o\left(n^{d}\right)$ ) with the property that every tight convex set $K$ induces at subset $\Pi_{K \text {,rich }}:=\left(\begin{array}{c}P_{K} \\ d\end{array}\right) \cap \Pi_{\text {rich }}$ of cardinality roughly $\epsilon^{d} n^{d}$.

In Section 5.5 we construct a net $N_{\text {loose }}$ for the complementary family $\mathcal{K}_{\text {loose }}^{+}$. The definition of $N_{\text {loose }}$ will overly resemble that of $N_{\text {sparse }}$ in Section 5.2 , and its analysis will closely follow the proof of Proposition 5.4. Informally, for the purpose of our 1-dimensional reduction we replace the points of $P_{K}$ with the vertices of certain clipped simplices that enclose these the points of $P_{K}$, which results in a more favourable density parameter.

The sub-family $\mathcal{K}^{-}$is treated in a fully symmetric manner (e.g., by reversal of the orientation of the $x_{d}$-axis).

\subsection{Piercing the tight sets}

In this section we construct a net $N_{\text {tight }}$ for the family $\mathcal{K}_{\text {tight }}^{+}$, which consists of all the tight sets in $\mathcal{K}^{+}$. To that end, we design a relatively sparse subset $\Pi_{\text {tight }} \subseteq\left(\begin{array}{l}P \\ d\end{array}\right)$ which encompasses a large fraction of the simplices of $\left(\begin{array}{c}P_{K} \\ d\end{array}\right)$, for every tight set $K \in \mathcal{K}_{\text {tight }}^{+}$.

Notation. Denote

$$
\Lambda:=\bigcup_{Q_{i, j} \in \mathcal{Q}(s, t)}\left(\begin{array}{c}
Q_{i, j} \\
d
\end{array}\right) .
$$


That is, $\Lambda$ is comprised of all the simplices in $\left(\begin{array}{l}P \\ d\end{array}\right)$ whose respective vertices come from the same part $Q_{i, j} \in \mathcal{Q}(s, t)$.

For each $p \in P$ let $\Pi(p) \subset\left(\begin{array}{l}P \\ d\end{array}\right)$ denote the subset of all the simplices $\pi \in\left(\begin{array}{l}P \\ d\end{array}\right)$ that are adjacent to $p$ (so that $p \in \pi$ ), and let $\Lambda(p):=\Pi(p) \cap \Lambda$ denote the smaller subset that is comprised of all the simplices $\mu \in \Lambda$ that are adjacent to $p$.

Note that every simplex $\pi \in \Pi(p)$ is of the form $\operatorname{conv}\left(\{p\} \cup \pi_{p}\right)$, where $\pi_{p} \in\left(\begin{array}{c}P \\ d-1\end{array}\right)$ is a $(d-2)$ dimensional simplex. Also note that all the simplices of $\Lambda(p)$ come from the ambient set $Q_{i, j}$ of $p$, so their number is $|\Lambda(p)|=\left(\begin{array}{c}\left|Q_{i, j}\right| \\ d-1\end{array}\right)$.

Definition. For every $p \in P$ we define a relation between the subsets $\Lambda(p)$ and $\Pi(p)$ of the simplices that are adjacent to $p$. We say that the simplices $\mu \in \Lambda(p)$ and $\pi=\operatorname{conv}\left(p \cup \pi_{p}\right) \in \Pi(p)$ are friendly at $p$ if all the $d-1$ vertices of the $(d-2)$-simplex $\pi_{p}:=\operatorname{conv}\left(p_{1}, \ldots, p_{d-1}\right)$ lie in the zone of $H(\mu)$ within the partition $\mathcal{Q}(s, t)$ (so that $H(\mu)$ crosses their ambient simplices $\varphi\left(p_{i}\right)$, for $1 \leq i \leq d-1$, which need not be distinct).

The hypergraph $\Pi_{\text {tight }}$. Let $p \in P$. We say that a simplex $\pi \in \Pi(p)$ is rich (at $p$ ) if there exist at least

$$
C_{\text {rich }} \cdot \frac{\delta^{d-1} \epsilon^{d-1} n^{d-1}}{s^{d-1} t^{(d-1)^{2} / d}}=\Theta\left(\left(\begin{array}{c}
\delta \epsilon n /\left(s t^{1-1 / d}\right) \\
d-1
\end{array}\right)\right),
$$

simplices $\mu \in \Lambda(p)$ that are friendly with $\pi$ at $p$. Here $C_{\text {rich }}>0$ is a sufficiently small constant that will be determined in the end of this analysis.

For each point $p \in P$ we add to $\Pi_{\text {rich }}$ every simplex $\pi \in \Pi(p)$ that is rich at $p$.

The cardinality of $\Pi_{\text {rich }}$. We bound the cardinality of $\Pi_{\text {rich }}$ in terms of the smaller quantity

$$
|\Lambda|=O\left(\sum_{i \in[s], j \in[t]}\left(\begin{array}{c}
n /(s t) \\
d
\end{array}\right)\right)=O\left(\frac{n^{d}}{(s t)^{d-1}}\right) .
$$

To that end, we describe a charging scheme which assigns every $\pi \in \Pi_{\text {rich }}$ to a number of simplices of $\Lambda$. Namely, for each point $p \in P$ we assign every rich $\pi \in \Pi(p)$ (which is then included in $\Pi_{\text {rich }}$ ) to every simplex $\mu \in \Lambda(p)$ that is friendly with $\pi$ at $p$, so each of these latter simplices $\mu$ pays 1 unit of charge to $\pi$.

By definition, $\pi$ is assigned to $\Omega\left(\frac{\delta^{d-1} \epsilon^{d-1} n^{d-1}}{s^{d-1} t^{(d-1)^{2} / d}}\right)$ simplices $\mu \in \Lambda(p)$, via at least one of its vertices $p$, so it suffices to bound the number of simplices in $\Pi_{\text {rich }}$ that can be assigned to any given $\mu \in \Lambda$.

Proposition 5.6. Any simplex $\mu \in \Lambda$ is assigned in the above manner a total of $O\left(n^{d-1} / t^{1-1 / d}\right)$ simplices of $\Pi_{\text {rich }}$.

Proof. Fix $\mu \in \Lambda$. Notice that every simplex $\pi \in \Pi_{\text {rich }}$ that is assigned to $\mu$, shares with it exactly one vertex $p \in P$, whilst the other $d-1$ vertices of $\pi$ must come from the zone of $H(\mu)$ within $\mathcal{Q}(s, t)$. However, by the virtues of the simplicial partition $\mathcal{Q}(s, t)$, the number of the latter vertices is at most $O\left(s \times \frac{n}{s t} \times t^{1-1 / d}\right)=O\left(n / t^{1 / d}\right)$. Thus, the number of the possible choices of $\pi$ cannot exceed

$$
O\left(\left(\begin{array}{c}
n / t^{1 / d} \\
d-1
\end{array}\right)\right)=O\left(n^{d-1} / t^{1-1 / d}\right) .
$$


Proposition 5.7. We have that

$$
\left|\Pi_{\text {rich }}\right|=O\left(\frac{n^{d}}{(\delta \epsilon)^{d-1} t^{2-2 / d}}\right)
$$

Proof. By Proposition 5.6, the simplices of $\Lambda$ pay a total of

$$
O\left(|\Lambda| \frac{n^{d-1}}{t^{(d-1) / d}}\right)=O\left(\frac{n^{2 d-1}}{s^{d-1} t^{d-1 / d}}\right)
$$

units of charge to the simplices of $\Pi_{\text {rich }}$. Since any simplex of $\Pi_{\text {rich }}$ receives at least

$$
C_{\text {rich }} \cdot \frac{(\delta \epsilon n)^{d-1}}{s^{d-1} t^{(d-1)^{2} / d}}
$$

of these units of charge, we have that

$$
\left|\Pi_{\text {rich }}\right|=O\left(\frac{n^{2 d-1}}{s^{d-1} t^{d-1 / d}} \cdot \frac{s^{d-1} t^{(d-1)^{2} / d}}{(\delta \epsilon n)^{d-1}}\right)=O\left(\frac{n^{d}}{(\delta \epsilon)^{d-1} t^{2-2 / d}}\right)
$$

Notice that $\left|\Pi_{\text {rich }}\right|=o\left(n^{d}\right)$ as long as $\frac{1}{\epsilon^{d / 2+\gamma}} \ll t$, for an arbitrary small albeit fixed, constant $\gamma>0$. It, therefore, remains to show that, for any convex set $K \in \mathcal{K}_{\text {tight }}^{+}$, a large fraction of the simplices in $\left(\begin{array}{c}P_{K} \\ d\end{array}\right)$ belong to $\Pi_{\text {rich }}$, so that Theorem 3.7 can be duly invoked.

Proposition 5.8. For any small enough choice of $C_{\text {rich }}>0$, we have that any tight set $K \in \mathcal{K}_{\text {tight }}^{+}$ induces a subset of hyperedges $\Pi_{K, \text { rich }}:=\left(\begin{array}{c}P_{K} \\ d\end{array}\right) \cap \Pi_{\text {rich }}$ of cardinality

$$
\left|\Pi_{K, \text { rich }}\right|=\Omega\left((\delta \epsilon n)^{d} / s^{d}\right) .
$$

Proof. Recall that the convex set $K$ is endowed with the characteristic $(d+1)$-tuple $\mathcal{T}(K)=$ $\left(P_{1}, \ldots, P_{d+1}\right)$, the subset $S_{K} \subset P_{K} \cap\left(\bigcup_{i=1}^{d} P_{i}\right)$, and the collections $\mathcal{Q}_{K \text {,tight }} \subset \mathcal{Q}_{K} \subset \mathcal{Q}_{d+1}(t)$. In addition, $K$ comes with the subset $Q_{K} \subseteq P_{K} \cap\left(\bigcup \mathcal{Q}_{K}\right) \subseteq P_{K} \cap P_{d+1}$. Furthermore, the cardinality of each of the above subsets $S_{K}$ and $Q_{K}$ is $\Theta(\delta \epsilon n / s)$.

As every part $Q_{j} \in \mathcal{Q}_{K}$ is full, it cut outs the subset $Q_{K, j} \subseteq Q_{K}$ of cardinality $\Omega\left(\delta \epsilon n /\left(s t^{1-1 / d}\right)\right)$. By definition, at least $\left|Q_{K}\right| / 2$ points in $Q_{K}$ belong to $\bigcup \mathcal{Q}_{K, \text { tight }}$.

Fix a tight part $Q_{j} \in \mathcal{Q}_{K, \text { tight }}$. Then at least $\left|\Lambda_{K, j} \times \Sigma_{K}\right| / 4$ of the pairs $(\mu, \tau) \in \Lambda_{K, j} \times \Sigma_{K}$ are tight. (In addition, recall that $\Sigma_{K} \subseteq\left(\begin{array}{c}S_{K} \\ d\end{array}\right)$ and $\left|\Sigma_{K}\right|=\Theta\left(\left|S_{K}\right|^{d}\right)=\Theta\left((\delta \epsilon n / s)^{d}\right)$.)

We first argue that there exist $\Omega\left(\left|\Lambda_{K, j}\right|\right)=\Omega\left(\left|Q_{K, j}\right|^{d}\right)$ simplices $\mu \in \Lambda_{K, j}$ so that each of them is friendly with $\Omega\left(\left|S_{K}\right|^{d-1}\right.$ ) simplices of $Q_{K, j} *\left(\begin{array}{c}S_{K} \\ d-1\end{array}\right)$ (at a common vertex of $Q_{K, j}$ ). To this end, for each $\mu \in \Lambda_{K, j}$ we consider the subset $\Sigma_{K}(\mu) \subseteq \Sigma_{K}$ of all the simplices $\tau \in \Sigma_{K}$ so that the pair $(\mu, \tau)$ is tight. By the pigeonhole principle, one can choose a sufficiently small constant $C>0$ (that depends only on the dimension $d$ ) so that the following holds: There is a subset $\Lambda_{K, j}^{*} \subseteq \Lambda_{K, j}$ of cardinality at least $C\left|\Lambda_{K, j}\right|$ so that every $\mu \in \Lambda_{K, j}^{*}$ satisfies $\left|\Sigma_{K}(\mu)\right| \geq C\left|\Sigma_{K}\right|=\Omega\left(\left|S_{K}\right|^{d}\right)$.

For each $\mu \in \Lambda_{K, j}^{*}$, we examine the subset $S_{\mu} \subseteq S_{K}$ of all the points in $S_{K}$ that belong to the zone of $H(\mu)$ in $\mathcal{Q}(s, t)$. We claim that $\left|S_{\mu}\right|=\Omega\left(\left|S_{K}\right|\right)$. Indeed, by the definition of tightness, at least one vertex in each $\tau \in \Sigma_{K}(\mu)$ must belong $S_{\mu}$ (so $S_{\mu}$ is a vertex transversal for $\Sigma_{K}(\mu)$ ). Since 
any point of $S_{\mu}$ is adjacent to at most $\left(\begin{array}{c}\left|S_{K}\right| \\ d-1\end{array}\right)$ simplices of $\Sigma_{K}(\mu)$, at least a fixed fraction of the points of $S_{K}$ must belong to $S_{\mu}$.

Note that each simplex $\mu=\operatorname{conv}\left(p_{1}, \ldots, p_{d}\right) \in \Lambda_{K, j}^{*}$ is friendly with all the $\Omega\left(\left|S_{\mu}\right|^{d-1}\right)=$ $\Omega\left(\left|S_{K}\right|^{d-1}\right)$ simplices of the form $\pi_{i}:=\operatorname{conv}\left(\left\{p_{i}\right\} \cup \kappa\right)$, for $\kappa \in\left(\begin{array}{c}S_{\mu} \\ d-1\end{array}\right)$ (each time at the respective point $p_{i}$ ).

For each point $p \in Q_{K, j}$ let $\Lambda_{K, j}^{*}(p) \subset \Lambda(p)$ denote the subset of all the simplices in $\Lambda_{K, j}^{*}$ that are adjacent to $p$. By the pigeonhole principle, there is a subset $Q_{K, j}^{*} \subset Q_{K, j}$ of cardinality $\Omega\left(\left|Q_{K, j}\right|\right)$ so that every $p \in Q_{K, j}^{*}$ satisfies $\left|\Lambda_{K, j}^{*}(p)\right| \geq C\left(\begin{array}{c}\left|Q_{K, j}\right| \\ d-1\end{array}\right)$ with a sufficiently small choice of the constant $C>0$.

Fix a point $p \in Q_{K, j}^{*}$. Since (i) $p$ is adjacent to $\Omega\left(\left|S_{K}\right|^{d-1}\right)$ simplices of $\{p\} *\left(\begin{array}{c}S_{K} \\ d-1\end{array}\right) \subset \Pi(p)$, and (ii) every simplex $\mu \in \Lambda_{K, j}^{*}(p)$ is friendly at $p$ with $\Omega\left(\left|S_{K}\right|^{d-1}\right)$ simplices of $\{p\} *\left(\begin{array}{c}S_{K} \\ d-1\end{array}\right)$, the pigeonhole principle yields a subset $\Pi^{*}(p) \subset\{p\} *\left(\begin{array}{c}S_{K} \\ d-1\end{array}\right)$ so that every $\pi \in \Pi^{*}(p)$ is friendly at $p$ with $\Omega\left(\left|\Lambda_{K, j}^{*}(p)\right|\right)=\Omega\left(\left|Q_{K, j}\right|^{d-1}\right)$ simplices of $\Lambda_{K, j}^{*}(p) \subset \Lambda(p)$.

Since the latter quantity $\left|Q_{K, j}\right|^{d-1}$ is

$$
\Omega\left(\left(\frac{\delta \epsilon n}{s t^{1-1 / d}}\right)^{d-1}\right)
$$

a suitably small choice of the constant $C_{\text {rich }}>0$ in the definition of $\Pi_{\text {rich }}$ guarantees that every simplex $\pi \in \Pi^{*}(p) \subset \Pi(p)$ is rich at $p$, and therefore belongs to $\Pi_{\text {rich }}$.

Repeating the above argument for each $p \in Q_{K, j}^{*}$ yields a subset

$$
\Pi_{K, j}^{*}:=\bigcup_{p \in Q_{K, J}^{*}} \Pi^{*}(p) \subseteq Q_{K, j} *\left(\begin{array}{c}
S_{K} \\
d-1
\end{array}\right)
$$

of cardinality $\Omega\left(\left|Q_{K, j}\right|\left|S_{K}\right|^{d-1}\right)$, which is entirely contained in $\Pi_{K, \text { rich }}=\Pi_{\text {rich }} \cap\left(\begin{array}{c}P_{K} \\ d\end{array}\right)$.

Finally, using that

$$
\sum_{j: Q_{j} \in \mathcal{Q}_{K, \text { tight }}}\left|Q_{K, j}\right| \geq\left|Q_{K}\right| / 4
$$

yields that the union $\bigcup_{Q_{j} \in \mathcal{Q}_{K, \text { tight }}} \subseteq \Pi_{K, \text { rich }}$ has cardinality

$$
\Omega\left(\left|Q_{K}\right|\left|S_{K}\right|^{d-1}\right)=\Omega\left((\delta \epsilon n / s)^{d}\right) .
$$

By Proposition 5.8, every tight set $K \in \mathcal{K}_{\text {tight }}^{+}$belongs to the family $\mathcal{K}\left(P, \Pi_{\text {rich }}, \epsilon, \sigma_{\text {rich }}\right)$, where $\sigma_{\text {rich }}=\Omega\left((\delta / s)^{d}\right)$. Plugging this (along with the bound of Proposition 5.7) into Theorem 3.7 yields the following result.

Theorem 5.9. Let $0<\epsilon, \sigma<1, P$ be an n-point set, $\Pi \subseteq\left(\begin{array}{l}P \\ d\end{array}\right), \delta>0$, and $\sigma_{\text {rich }}=\Theta\left(\delta^{d} / s^{d}\right)$ be as above. Let $\mathcal{K}_{\text {tight }}^{+} \subseteq \mathcal{K}^{+} \subseteq \mathcal{K}_{\text {surr }}$ be the subfamily of all the tight sets as defined in Section 5.3. Then for every $u>0$ one can pierce $\mathcal{K}_{\text {tight }}^{+}$using a net $N_{\text {tight }}$ of cardinality

$$
\left|N_{\text {tight }}\right|=O\left(\frac{u^{(d-1)^{2}}}{\left(\sigma_{\text {rich }}\right)^{\beta_{d-1}} \delta^{d-1}} \cdot \frac{1}{\epsilon^{2 d-1} t^{2-2 / d}}+u \cdot f\left(\left(\sigma_{\text {rich }}\right)^{\beta_{d-1}} u^{1 /(d-1)} \epsilon\right)\right) .
$$


To establish a favourable recurrence for $f(\epsilon)$, in what follows we choose $u \ggg s \ggg 1 / \delta$ to be arbitrary small albeit fixed degrees of $1 / \epsilon$, and $1 / \epsilon^{d / 2} \ll t \ll 1 /\left(\epsilon^{d-1 / 2} s\right)$, so the first term becomes $o\left(1 / \epsilon^{d}\right)$, and the second term is dominated by $u \cdot f\left(\epsilon \cdot u^{1 /(d-1)+\gamma^{\prime}}\right)$, for an arbitrary constant $\gamma^{\prime}>0$.

\subsection{Piercing the loose sets}

In this section we construct a net $N_{\text {loose }}$ for the sub-family $\mathcal{K}_{\text {loose }}^{+}$which encompasses all the loose sets in $\mathcal{K}^{+}$. Our net overly resembles the auxiliary net $Q_{\text {sparse }}$ of Section 5.2 , so its analysis loosely follows the proof of Proposition 5.4.

The net $N_{\text {loose }}$. Fix $1 \leq i \leq s$. We consider the secondary vertical simplicial partition $\mathcal{P}_{i}(u)$ of the set $P_{i}$, and the set $\mathcal{L}_{i}(u)$ of at most $u^{(d-1)^{2}}$ lines, as introduced in Section 5.2 . As before, for each $p \in P_{i}$ we use $\varphi(p)$ to denote the ambient simplex of $p$ in the $t$-partition $\mathcal{Q}_{i}(t)$ of $P_{i}$. However, this time around it is enough to clip $\varphi(p)$ to the vertical prism above the ambient simplex $\Delta_{i}$ of $p$ in $\mathcal{P}(s)$. Let $\hat{\varphi}(p)$ denote the resulting finer analogue of $\varphi(p)$. Clearly, the polytope $\hat{\varphi}(p)$ is delimited by at most $2 d+1$ facets. Denote $\hat{\Phi}_{i}:=\left\{\hat{\varphi}(p) \mid p \in P_{i}\right\}$, and let $\hat{V}_{i}$ denote the set of all the vertices of the clipped simplices of $\hat{\Phi}_{i}$.

Denote $\hat{V}=\bigcup_{1 \leq i \leq s} \hat{V}_{i}$. Clearly, we have that $|\hat{V}|=O(s t)$.

For each $1 \leq i \leq s$, and each $\ell \in \mathcal{L}_{i}(u)$, we construct a subset $\hat{X}_{\ell}=\left\{\mu \cap \ell \mid \mu \in\left(\begin{array}{c}\hat{V} \\ d\end{array}\right)\right\}$ of all the $\ell$-intercepts of the simplices of $\left(\begin{array}{c}\hat{V} \\ d\end{array}\right)$. Note that $\left|\hat{X}_{\ell}\right|=O\left(s^{d} t^{d}\right)$. We then obtain a local net $\hat{N}_{\ell}$ which includes every $\left\lceil C_{\text {loose }} t^{d-1-1 /(2 d-1)}\right\rceil$-th point of $\hat{X}_{\ell}$; here $C_{\text {loose }}>0$ is a suitably small constant that will be determined so as to suit Theorem 5.10. We then define

$$
N_{\text {loose }}:=\bigcup_{1 \leq i \leq s} \bigcup_{\ell \in \mathcal{L}_{i}(u)} \hat{N}_{\ell}
$$

Note that our net has cardinality

$$
\left|N_{\text {loose }}\right|=O\left(s u^{(d-1)^{2}} \frac{\left|\hat{X}_{\ell}\right|}{C_{\text {loose }} t^{d-1-1 /(2 d-1)}}\right)=O\left(s^{d+1} u^{(d-1)^{2}} t^{1+1 /(2 d-1)}\right) .
$$

It remains to show that $N_{\text {loose }}$ pierces every set $K \in \mathcal{K}_{\text {loose }}^{+}$.

Theorem 5.10. The following property holds for any small enough constant $C_{\text {loose }}>0$.

Let an n-point set $P, \Pi \subseteq\left(\begin{array}{l}P \\ d\end{array}\right), 0<\epsilon, \sigma \leq 1,0<\delta \leq 1$, and the families $\mathcal{K}_{\text {loose }}^{+} \subseteq \mathcal{K}_{\text {surr }} \subseteq$ $\mathcal{K}(P, \Pi, \epsilon, \sigma)$ be as defined in Sections 5.2 and 5.3. Then every set in $\mathcal{K}_{\text {loose }}^{+}$is pierced by the net $N_{\text {loose }}$.

Proof. Fix a loose convex set $K \in \mathcal{K}_{\text {loose }}^{+}$. Recall that $K$ is endowed with the characteristic vertically regular $(d+1)$-tuple $\mathcal{T}(K)=\left(P_{1}, \ldots, P_{d+1}\right)$, so that $\Delta_{d+1} \supset P_{d+1}^{\perp}$ is surrounded by the remaining $d$ simplices $\Delta_{i} \supset P_{i}^{\perp}$, for $1 \leq i \leq d$. Furthermore, $K$ comes with subsets $Q_{K} \subseteq P_{K} \cap P_{d+1}$ and $S_{K} \subseteq P_{K} \cap\left(\bigcup_{i=1}^{d} P_{i}\right)$, and determines the set of simplices

$$
\Sigma_{K}:=\left\{\operatorname{conv}\left(p_{1}, p_{2}, \ldots, p_{d}\right) \mid p_{1} \in S_{K} \cap P_{1}, p_{2} \in S_{K} \cap P_{2}, \ldots, p_{d} \in S_{K} \cap P_{d}\right\} .
$$

so that $\left|S_{K} \cap P_{i}\right|=\Theta\left(\delta\left|P_{K}\right| / s\right)$ for every $1 \leq i \leq d+1$, and every point of $Q_{K}$ lies above every simplex of $\Sigma_{K}$. Specifically, the set $Q_{K} \subseteq P_{K}$ is contained in the union $\bigcup \mathcal{Q}_{K}$ of the full parts $Q_{j}$ in the partition $\mathcal{Q}_{d+1}(t)$ of $P_{d+1}$. We will also use that $K$ is not $\epsilon^{*}$-crowded in $\mathcal{Q}(s, t)$, where $\epsilon^{*}=C^{*} \cdot \delta \epsilon /\left(s t^{1-2 /(2 d-1)}\right)$. 
By the choice of $K \in \mathcal{K}_{\text {loose }}^{+}$, at least half of the points of $Q_{K}$ belong to the sub-collection $\mathcal{Q}_{K, \text { loose }}$ of the loose parts $Q_{j} \in \mathcal{Q}_{K}$, each of them attaining at least $\left|\Lambda_{K, j}\right|\left|\Sigma_{K}\right| / 4$ loose pairs $(\mu, \tau) \in \Lambda_{K, j} \times \Sigma_{K}$

For every $\tau \in \Sigma_{K}$, and every part $Q_{j} \in \mathcal{Q}_{K, \text { loose }}$, let $\Psi_{K}\left(\tau, Q_{j}\right)$ denote the subset of all the loose pairs in $\Sigma_{K} \times \Lambda_{K, j}$ that involve $\tau$. For every simplex $\tau \in \Sigma_{K}$ we use $\mathcal{Q}_{K, \tau} \subseteq \mathcal{Q}_{K \text {,loose }}$ to denote the subset of all the parts $Q_{j} \in \mathcal{Q}_{K, \text { loose }}$ so that $\left|\Psi_{K}\left(\tau, Q_{j}\right)\right| \geq\left|\Lambda_{K, j}\right| / 4$.

We say that a simplex $\tau \in \Sigma_{K}$ is good if the parts of $\mathcal{Q}_{K, \tau}$ encompass at least $\left|Q_{K}\right| / 16$ points of $Q_{K}$, and we say that $\tau$ is bad otherwise.

Proposition 5.11. At least $\left|\Sigma_{K}\right| / 16$ of the simplices in $\Sigma_{K}$ are good.

Proof of Proposition 5.11. Assume for a contradiction that fewer than $\left|\Sigma_{K}\right| / 16$ of the simplices in $\Sigma_{K}$ are good. We uniformly sample a pair $(\tau, p) \in \Sigma_{K} \times Q_{K}$, and claim that $p$ belongs to a part of $\mathcal{Q}_{K, \tau}$ with probability at least $1 / 8$. To see this, it is sufficient to note that (1) $p$ belongs to a part $Q_{j}$ of $\mathcal{Q}_{K, \text { loose }}$ with probability at least $1 / 2$, and (2) for any fixed $Q_{j} \in \mathcal{Q}_{K, \text { loose }}$, we have that $\left|\Psi_{K}\left(\tau, Q_{j}\right)\right|>\left|\Lambda_{K, j}\right| / 4$ with probability at least $1 / 4$.

The above calculation implies that, for a randomly and uniformly chosen $\tau \in \Sigma_{K}$, the expected cardinality of the set $Q_{K} \cap\left(\bigcup \mathcal{Q}_{K, \tau}\right)$ is at least $\left|Q_{K}\right| / 8$. Thus, by the pigeonhole principle, at least $\left|\Sigma_{K}\right| / 16$ simplices $\tau \in \Sigma_{K}$ must satisfy

$$
\left|Q_{K} \cap\left(\bigcup \mathcal{Q}_{K, \tau}\right)\right| \geq\left|Q_{K}\right| / 16
$$

and are therefore good. This completes the proof of Proposition 5.11 .

Let $\tau=\operatorname{conv}\left(p_{1}, \ldots, p_{d}\right) \in \Sigma_{K}$ be a simplex so that $p_{i} \in S_{K} \cap P_{i}$ for all $1 \leq i \leq d$. We assign $\tau$ to a unique simplex $\phi(\tau) \in\left(\begin{array}{l}\hat{V} \\ d\end{array}\right)$. To this end, we recall that the vertices of $\tau$ determine $d$ ambient clipped simplices $\hat{\varphi}\left(p_{i}\right) \in \hat{\Phi}_{i}$, for $1 \leq i \leq d$. Note that the convex hull $U=\operatorname{conv}\left(\bigcup_{i=1}^{d} \hat{\varphi}\left(p_{i}\right)\right)$ has a unique facet $\hat{\tau}$ so that $Q_{K}^{\perp} \subset(\hat{\tau})^{\perp}$, and $U$ lies below $H(\hat{\tau})$. Indeed, since the simplex $\Delta_{d+1} \supset Q_{K}^{\perp}$ is surrounded by the ambient simplices $\Delta_{i} \supset\left(\hat{\varphi}\left(p_{i}\right)\right)^{\perp}$ of the $d$ parts $P_{i}$ of the characteristic $(d+1)$ tuple $\mathcal{T}(K)$, for $1 \leq i \leq d$, the $d$ clipped simplices $\hat{\varphi}\left(p_{i}\right)$ must have pairwise disjoint projections. Therefore, their convex hull $U$ must be bounded from above by a hyperplane $H=\operatorname{aff}\left(v_{1}, \ldots, v_{d}\right)$, that is supported by $d$ respective vertices $v_{i}$ which come the distinct clipped simplices $\hat{\varphi}\left(p_{i}\right)$, for $1 \leq i \leq d$. Thus, the desired facet $\hat{\tau}$ of $U$ is given by $\operatorname{conv}\left(v_{1}, \ldots, v_{d}\right)$. We then assign $\tau$ to $\phi(\tau):=\hat{\tau}=\operatorname{conv}\left(v_{1}, \ldots, v_{d}\right)$.

Proposition 5.12. Let $\tau \in \Sigma_{K}$ be a good simplex. Then there exist $\Omega\left(\left|Q_{K}\right|\right)$ points in $Q_{K}$ that lie above $\phi(\tau)$.

Proof of Proposition 5.12. We argue that every part $Q_{j} \in \mathcal{Q}_{K, \tau}$ contributes $\Theta\left(\left|Q_{K, j}\right|\right)$ points of $Q_{K, j}$ that lie above $\phi(\tau)$. To that end, we fix $Q_{j} \in \mathcal{Q}_{K, \tau}$. The crucial observation is that for every loose pair $(\tau, \mu) \in \Psi_{K}\left(\tau, Q_{j}\right)$, all the $d$ vertices of $\mu$ must lie above $\phi(\tau)$.

Indeed, fix a loose pair $(\tau, \mu) \in \Psi_{K}\left(\tau, Q_{j}\right)$ so that $\tau=\operatorname{conv}\left(p_{1}, \ldots, p_{d}\right)$, and $p_{i} \in S_{K} \cap P_{i}$ for all $1 \leq i \leq d$. Since $(\tau, \mu)$ is loose (and, in particular, it is a parallel pair), the hyperplane $H(\mu)$ lies above $\tau$. Moreover, $H(\mu)$ misses the the ambient clipped simplex $\hat{\varphi}\left(p_{i}\right)$ of every vertex of $\tau$; hence, $H(\mu)$ lies above each of these clipped simplices $\hat{\varphi}\left(p_{i}\right)$. Therefore, $U:=\operatorname{conv}\left(\bigcup_{i=1}^{d} \hat{\varphi}\left(p_{i}\right)\right)$ lies entirely below $H(\mu)$, so the simplex $\mu$ must lie above $U$. In particular, $\mu$ must lie above the facet $\phi(\tau)$ of $U$. 
Since $Q_{j} \in \mathcal{Q}_{K, \tau}$, at least $\left|\Psi_{K}\left(\tau, Q_{j}\right)\right| \geq\left|\Lambda_{K, j}\right| / 4=\left|\left(\begin{array}{c}Q_{K, j} \\ d\end{array}\right)\right| / 4$ simplices in the set $\Lambda_{K, j}=\left(\begin{array}{c}Q_{K, j} \\ d\end{array}\right)$ lie above $\phi(\tau)$. Hence, there exist $\Theta\left(\left|Q_{K, j}\right|\right)$ points that are adjacent to at least one of these simplices and, therefore, lie above $\phi(\tau)$.

Repeating the above argument for each $Q_{j} \in \mathcal{Q}_{K, \tau}$, and using that $\sum_{j \in[t]: Q_{j} \in \mathcal{Q}_{K, \tau}}\left|Q_{K, j}\right| \geq$ $\left|Q_{K}\right| / 16$ (because $\tau$ is a good simplex), yields at total of $\Omega\left(\left|Q_{K}\right|\right)$ points in $Q_{K}$ that lie above $\phi(\tau)$. This concludes the proof of Proposition 5.12.

Back to the proof of Theorem 5.10. Let $\Sigma_{K}^{\prime}$ denote the subset of all the good simplices in $\Sigma_{K}$. For each simplex $\tau \in \Sigma_{K}^{\prime}$ we fix (by the means Proposition 5.12) a subset $Q_{K}(\tau) \subset Q_{K}$ of $\Theta\left(\left|Q_{K}\right|\right)$ points that lie above the respective simplex $\phi(\tau)$. Denote $\Xi_{K}(\tau):=\left(\begin{array}{c}Q_{K}(\tau) \\ d\end{array}\right)$. Note that any vertical line that crosses a simplex $\pi \in \Xi_{K}(\tau)$, must meet all the three simplices $\tau, \phi(\tau)$ and $\pi$, in this increasing order.

For each simplex $\pi \in\left(\begin{array}{c}Q_{K} \\ d\end{array}\right)$ we consider the subset

$$
\Sigma(\pi):=\left\{\tau \in \Sigma_{K}^{\prime} \mid \pi \in \Xi_{K}(\tau)\right\} .
$$

Using Proposition 5.11, and that $\left|\Xi_{K}(\tau)\right|=\Theta\left(\left|Q_{K}\right|^{d}\right)$ for every good simplex $\tau \in \Sigma_{K}^{\prime}$, a simple double-counting argument yields a subset $\Xi_{K} \subseteq\left(\begin{array}{c}Q_{K} \\ d\end{array}\right)$ of $\Omega\left(\left|Q_{K}\right|^{d}\right)$ simplices $\pi \in\left(\begin{array}{c}Q_{K} \\ d\end{array}\right)$ so that $|\Sigma(\pi)|=\Omega\left(\left|\Sigma_{K}\right|\right)$.

Since $\left|Q_{K}\right|=\Theta(\delta \epsilon n / s)$, the set $K$ is $\Omega(\delta \epsilon)$-heavy with respect to the point set $P_{d+1}$. Since $K$ is $\tilde{\epsilon}_{n a r}$-spread in the secondary vertical partition $\mathcal{P}_{d+1}(u)$ of $P_{d+1}$, and given a suitably small choice of the narrowness constant $\tilde{C}_{n a r}>0$ in the beginning of Section 5.2 the set $\mathcal{L}_{i}(u)$ which we obtained via Theorem 3.3 must include a line $\ell$ that pierces a subset $\Xi_{K}(\ell) \subseteq \Xi_{K}$ of $\Omega\left((\delta \epsilon n / s)^{d}\right)$ simplices.

Fix a simplex $\pi \in \Xi_{K}(\ell)$ that is crossed by $\ell$. Then the set $\Sigma(\pi) \subseteq \Sigma_{K}$ contains $\Omega\left(\left|\Sigma_{K}\right|\right)$ simplices $\tau$ so that $\ell$ crosses their respective simplices $\phi(\tau)$ between $\ell \cap \tau$ and $\ell \cap \pi$. In particular, for each $\tau \in \Sigma(\pi)$, the interval $K \cap \ell$ contains the point $\phi(\tau) \cap \ell \in \hat{X}_{\ell}$.

Since no part $Q_{j} \in \mathcal{Q}_{K \text {,loose }}$ is $\epsilon^{*}$-crowded, any simplex $\hat{\tau}=\operatorname{conv}\left(v_{1}, \ldots, v_{d}\right) \in\left(\begin{array}{c}\hat{V}_{i} \\ d\end{array}\right)$ is assigned

$$
O\left(\left(\epsilon^{*} n\right)^{d}\right)=O\left(\left(\frac{\delta \epsilon n}{s t^{1-2 /(2 d-1)}}\right)^{d}\right)=O\left((\delta \epsilon n / s)^{d} \cdot \frac{1}{t^{d-1-1 /(2 d-1)}}\right)
$$

simplices $\tau \in \Sigma_{K}$ so that $\phi(\tau)=\hat{\tau}$.

Indeed, for any simplex $\tau=\operatorname{conv}\left(p_{1}, \ldots, p_{d}\right) \in \Sigma_{K}$ that is assigned to $\hat{\tau}=\operatorname{conv}\left(v_{1}, \ldots, v_{d}\right)$, and any $1 \leq i \leq d$, the vertex $p_{i} \in S_{K} \cap P_{i}$ must come from the unique part $Q_{i, j} \in \mathcal{Q}_{i}(t)$ whose respective clipped simplex has vertex $v_{i}$. Furthermore, there exist at most $\epsilon^{*} n$ possible choices of $p_{i} \in Q_{i, j}$, which amounts to $O\left(\left(\epsilon^{*} n\right)^{d}\right)$ such simplices $\tau \in \Sigma_{K}$. See Figure 21

Thus, the interval $K \cap \ell$ must contain

$$
\Omega\left(\frac{\left|\Xi_{K}(\ell)\right|}{(\delta \epsilon n / s)^{d}} \cdot t^{d-1-\frac{1}{2 d-1}}\right)=\Omega\left(t^{d-1-\frac{1}{2 d-1}}\right)
$$

points of $\hat{X}_{\ell}$. Thus, given a small enough constant $C_{\text {loose }}>0$, the interval $K \cap \ell$ must contain at least one point of the net $\hat{N}_{\ell} \subseteq N_{\text {loose }}$. This concludes the proof of Theorem 5.10. 


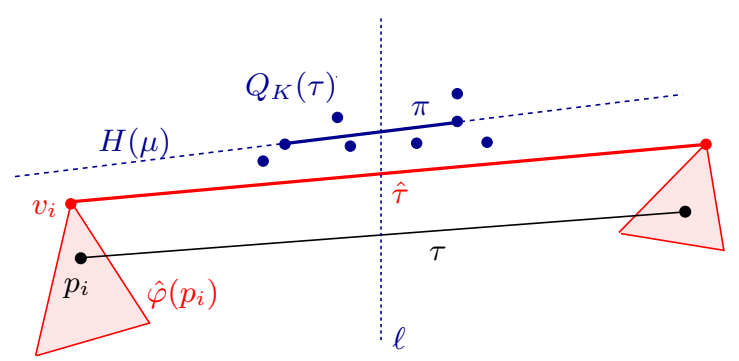

Figure 21: Assigning a simplex $\tau \in \Sigma_{K}$ to the simplex $\phi(\tau)=\hat{\tau}$, in $\left(\begin{array}{c}\hat{V} \\ d\end{array}\right)$. The set $Q_{K}(\tau) \subset Q_{K}$ consists of $\Omega\left(\left|Q_{K}\right|\right)$ points that lie above $\hat{\tau}$. Any line $\ell \in \mathcal{L}_{d+1}(u)$ that crosses a simplex $\pi \in\left({ }_{d}^{Q_{K}(\tau)}\right)$ must meet all three simplices $\tau, \hat{\tau}$, and $\pi$, in this increasing order of the $x_{d}$-coordinate.

\subsection{Piercing the sets of $\mathcal{K}_{\text {surr }}-$ Wrap-up}

Our net $N_{\text {surr }}$ for the remaining family $\mathcal{K}_{\text {surr }}$ is defined as the following combination of 7 nets

$$
N_{\text {surr }}:=N\left(\epsilon_{\delta}, s\right) \cup N_{\text {crowd }}\left(\epsilon^{*}\right) \cup N^{\triangle}(\hat{\epsilon}) \cup N^{\cup}\left(\tilde{\epsilon}_{\text {nar }}, u\right) \cup N_{\text {sparse }} \cup N_{\text {tight }} \cup N_{\text {loose }} \text {. }
$$

By definition (and using Theorems 5.9 and 5.10), the net $N_{\text {surr }}$ pierces every convex set in $\mathcal{K}_{\text {surr }}$. To bound the cardinality of $N_{\text {surr }}$, we combine the upper bounds (21) in Section 5.1, (23), (24), (25), and (26) in Section 5.2, (28) in Section 5.4, and (29) in Section 5.5

This yields

$$
\begin{aligned}
& \left|N_{\text {surr }}\right| \leq s \cdot f\left(\delta \epsilon \cdot s^{1 /(d-1)}\right)+s \cdot t \cdot f\left(\epsilon \delta \cdot t^{2 /(2 d-1)}\right)+s \cdot u \cdot f\left(u^{1 /(d-1)} \cdot \delta \epsilon\right)+u \cdot f\left(\left(\sigma_{\text {rich }}\right)^{\beta_{d-1}} u^{1 /(d-1)} \epsilon\right)+ \\
& \quad+O\left(\frac{s t^{1-1 / d}}{\delta \epsilon} \log \frac{1}{\epsilon}+s \cdot u^{(d-1)^{2}+d} \cdot t+\frac{u^{(d-1)^{2}}}{\left(\sigma_{\mathrm{rich}}\right)^{\beta_{d-1}} \delta^{d-1}} \cdot \frac{1}{\epsilon^{2 d-1} t^{2-2 / d}}+s^{d+1} u^{(d-1)^{2}} t^{1+1 /(2 d-1)}\right)
\end{aligned}
$$

so that in the 6 th and 7 th summands, we have $\sigma_{\text {rich }}=\Theta\left((\delta / s)^{d}\right)$.

Recall that the parameters $1 / \delta \lll s \lll u$ are arbitrary small, albeit fixed, degrees of $1 / \epsilon$, whereas $t$ is a fixed degree of $1 / \epsilon$ which will be chosen so as to establish the best possible recurrence for the quantity $\left|N_{\text {surr }}\right|$.

Indeed, substituting into 31

$$
t:=1 / \epsilon^{\frac{d(2 d-1)^{2}}{6 d^{2}-6 d+2}}
$$

renders the 5 th through 8 th (non-recursive) summands $O\left(1 / \epsilon^{d-1 / 2}\right)$, provided that $d \geq 3$. Hence, we obtain the following result.

Theorem 5.13. With the previous definitions, the net $N_{\text {surr }}$ in (30) pierces every convex set in the family $\mathcal{K}_{\text {surr }}=\mathcal{K}(P, \Pi, \epsilon, \sigma) \backslash \mathcal{K}_{\mathrm{vcon}}$. Furthermore, its cardinality satisfies

$$
\begin{gathered}
\left|N_{\text {surr }}\right| \leq s \cdot f\left(\epsilon \cdot s^{1 /(d-1)-\gamma^{\prime}}\right)+t^{1+\gamma^{\prime}} \cdot f\left(\epsilon \cdot t^{\frac{2}{2 d-1}-\gamma^{\prime}}\right)+u^{1+\gamma^{\prime}} \cdot f\left(u^{1 /(d-1)-\gamma^{\prime}} \cdot \epsilon\right)+ \\
+O\left(1 / \epsilon^{d-1 / 2}\right)
\end{gathered}
$$


for any constant $\gamma^{\prime}>0$. Here $s$ and $u$ are integers that are proportional to arbitrary small (albeit, fixed and positive) degrees of $1 / \epsilon$ that satisfy $1 / \delta \lll s \lll u \lll 1 / \epsilon$, and $1 / \epsilon^{d / 2} \lll t=o\left(\epsilon^{d}\right)$ as above.

\section{The final recurrence and concluding remarks}

\subsection{The final recurrence for $f_{d}(\epsilon)$}

In this section we develop the complete recurrence for the quantity $f_{d}(\epsilon)=f(\epsilon)$, which solves to $f(\epsilon)=O\left(\frac{1}{\epsilon^{d-1 / 2+\gamma}}\right)$, for an arbitrary constant $\gamma>0$.

As mentioned in Section 2, we fix a suitably small constant $0<\epsilon_{0}<1$ and use the old bound $f(\epsilon)=O^{*}\left(\epsilon_{0}^{-d}\right)=O(1)$ of Chazelle et al. [22] whenever $\epsilon>\epsilon_{0}$. (The choice of $\epsilon_{0}$ will affect the multiplicative constant in the eventual asymptotic bound on $f(\epsilon)$.) Assume then that $\epsilon<\epsilon_{0}$.

Bounding $f(\epsilon, \rho, \sigma)$. To obtain the desired recursion for $f(\epsilon)=f(\epsilon, 1,1)$, we first express $f(\epsilon, \rho, \sigma)$ in terms of the simpler quantities $f\left(\epsilon^{\prime}\right)$, for $\epsilon^{\prime}>\epsilon$. To this end, we fix an arbitrary family $\mathcal{K}=$ $\mathcal{K}(P, \Pi, \epsilon, \sigma)$ that satisfies $|\Pi| /\left(\begin{array}{c}P \\ 2\end{array}\right) \leq \rho$. (As explained in Section 2.4 , we can also assume with no loss of generality that $|P| \geq 1 / \epsilon^{d-1 / 2}$.) Theorems 4.12 and 5.13 together yield a net $N:=N_{\text {vcon }} \cup N_{\text {surr }}$ for the entire family $\mathcal{K}$. Hence, it remains to bound the quantity $|N|$.

Assume first that $\rho>\epsilon^{d}$. Combining the bounds in Theorems 4.12 and 5.13 results in the following recurrence

$$
\begin{gathered}
f(\epsilon, \rho, \sigma) \leq f\left(\epsilon, \frac{\rho}{h}, \frac{\sigma}{2}\right)+s \cdot f\left(\epsilon \cdot s^{1 /(d-1)-\gamma^{\prime}}\right)+ \\
+l \cdot f(\epsilon \cdot l)+\sum_{i=0}^{l} 2^{i} \cdot r^{d-1 / 2} \cdot f\left(\epsilon \cdot 2^{2 i} \cdot r^{1-\gamma^{\prime}}\right)+ \\
+t^{1+\gamma^{\prime}} \cdot f\left(\epsilon \cdot t^{\frac{2}{2 d-1}-\gamma^{\prime}}\right)+u^{1+\gamma^{\prime}} \cdot f\left(u^{1 /(d-1)-\gamma^{\prime}} \cdot \epsilon\right)+ \\
+O\left(1 / \epsilon^{d-1 / 2}\right),
\end{gathered}
$$

where $h, r, s, u$ are proportional to arbitrary small, albeit fixed, degrees of $1 / \epsilon$ that satisfy $h \lll$ $r \lll s \lll u \lll 1 / \epsilon, t=o\left(1 / \epsilon^{d}\right)$ is as above, and $\Theta(\log 1 / \epsilon)=l \leq\left\lceil\frac{1}{2} \log r\right\rceil+1$, and $\gamma^{\prime}>0$ is an arbitrary small, albeit fixed, constant. (The choice $h, r, s, t, u$ and $\gamma^{\prime}>0$ depends on the value $\gamma>0$ in Theorem 1.1, via the ground constant $\gamma_{0}>0$ that is hidden by our $\lll$ notation; see Section 2.1.)

We keep the parameters $h, r, s, t, u$ fixed and let $F(\epsilon)$ denote the last six terms of (33), which do not depend on the density $\rho$ of $\Pi$. We thus rewrite the recurrence as

$$
f(\epsilon, \rho, \sigma) \leq f\left(\epsilon, \frac{\rho}{h}, \frac{\sigma}{2}\right)+F(\epsilon) .
$$

We begin with $f(\epsilon)=f(\epsilon, 1,1)$ and recursively apply the inequality (34), each time only to the "leading" term, which involves the density $\rho$. This recurrence in $\rho$ bottoms out when the value of 
$\rho$ falls below $\epsilon^{d}$. Since $h$ is a fixed (though arbitrary small) positive power of $1 / \epsilon$, the inequality (34) is applied $k=O\left(\log _{h} 1 / \epsilon^{d}\right)=O(1)$ times, which implies

$$
f(\epsilon)=O\left(F(\epsilon)+f\left(\epsilon, \sigma_{0}, \epsilon^{d}\right)\right)
$$

for a suitably small constant $\sigma_{0}>0$. Theorem 3.7 yields

$$
f\left(\epsilon, \sigma, \epsilon^{d}\right)=O\left(1 / \epsilon^{d-1}+r^{d-1} \cdot f\left(\epsilon \cdot \sigma^{\beta_{d-1}} \cdot r\right)\right)=O\left(1 / \epsilon^{d-1}+r^{d-1} \cdot f(\epsilon \cdot r)\right),
$$

which is clearly dominated by $F(\epsilon)$. Hence, we conclude that

$$
\begin{gathered}
f(\epsilon) \leq O\left(s \cdot f\left(\epsilon \cdot s^{1 /(d-1)-\gamma^{\prime}}\right)+l \cdot f(\epsilon \cdot l)+\sum_{i=0}^{l} 2^{i} \cdot r^{d-1 / 2} \cdot f\left(\epsilon \cdot 2^{2 i} \cdot r^{1-\gamma^{\prime}}\right)\right)+ \\
O\left(t^{1+\gamma^{\prime}} \cdot f\left(\epsilon \cdot t^{\frac{2}{2 d-1}-\gamma^{\prime}}\right)+u^{1+\gamma^{\prime}} \cdot f\left(u^{1 /(d-1)-\gamma^{\prime}} \cdot \epsilon\right)\right)+ \\
O\left(1 / \epsilon^{d-1 / 2}\right) .
\end{gathered}
$$

Here again $\Theta(\log 1 / \epsilon)=l \leq\left\lceil\frac{1}{2} \log r\right\rceil+1, \gamma^{\prime}>0$ is an arbitrary small constant, and $r \lll s \lll$ $u \lll 1 / \epsilon$ are arbitrary small degrees of $1 / \epsilon$, which can be controlled using the ground constant $0<\gamma_{0}$ that was introduced in the beginning of Section 2.1 .

Bounding $f(\epsilon)$. We emphasize that the final recurrence (35) does not involve $\rho$ and $\sigma$. Furthermore, each recursive term on its right hand side is bounded by $w^{a} \cdot f(\epsilon \cdot w)$, for $a \leq d-1 / 2+O\left(\gamma^{\prime}\right)$ (and the contribution of the second term is, in fact, "linear" in $1 / \epsilon$ ). Furthermore, the third summand is dominated by constantly many terms of the desired form $w^{a} \cdot f(\epsilon \cdot w)$, where $a \leq$ $d-1 / 2+O\left(\gamma^{\prime}\right)$ and $r \leq w \leq r^{2}$. This last recurrence terminates when $\epsilon \geq \epsilon_{0}$, in which case we have $f(\epsilon)=O(1)$.

By following the standard inductive approach which applies to recurrences of this type (see, e.g., [44], and also [34, 54] and [55, Section 7.3.2]), and fixing suitably small constants $\gamma_{0}<\gamma^{\prime}(<\gamma)$, the recurrence solves to

$$
f(\epsilon)=O\left(\frac{1}{\epsilon^{d-1 / 2+\gamma}}\right)
$$

where $\gamma>0$ is an arbitrary small constant, and the constant of proportionality depends on $\gamma$. (To establish the bound for a particular value of $\gamma>0$, the parameters $\gamma_{0}>0$ and $\gamma^{\prime}>0$ and, therefore, the positive powers of $1 / \epsilon$ in $h, r, s$, and $u$, are all set to be much smaller than $\gamma$. Furthermore, the value of $\epsilon_{0}$ is chosen so as to guarantee that $1 / \epsilon_{0}$ is much larger than these parameters. Note that the resulting constant of proportionality in $O(\cdot)$ may be exponential in $1 / \gamma$.)

This concludes the proof of Theorem 1.1 .

\subsection{Concluding remarks}

- Despite the superficial similarity between the bound of Theorem 1.1 and the author's planar bound $f_{2}(\epsilon)=O\left(1 / \epsilon^{3 / 2+\gamma}\right)$, for any constant $\gamma>0$ [51], the present analysis explicitly assumes that $d \geq 3$, and does not yield the planar bound. (Nevertheless, it can be easily adapted to show a weaker bound $f_{2}(\epsilon)=O\left(\epsilon^{a}\right)$, for some constant $3 / 2<a<2$.) 
- The author conjectures that the actual asymptotic behaviour of the functions $f_{d}(\epsilon)$ in any dimension $d \geq 1$ is close to $1 / \epsilon$, as is indeed the case for their "strong" counterparts with respect to simply shaped objects in $\mathbb{R}^{d}[35]$.

- The author anticipates that a major improvement of the upper bound on $f_{d}(\epsilon)$ (i.e., beyond $\left.\tilde{O}\left(1 / \epsilon^{d-1}\right)\right)$ will require stronger selection-type results and, in particular, a more "complete" solution of the Multiple Selection problem than given by Theorem 3.3 .

- The recently improved analysis of the transversal numbers $C_{d}(p, q)$ that arise in the HadwigerDebrunner problem (see Section 1), due to Keller, Smorodinsky, and Tardos [39], implies that

$$
C_{d}(p, q) \leq f_{d}\left(\Omega\left(p^{-1-\frac{d-1}{q-d}}\right)\right)
$$

Here, as before, $f_{d}(\epsilon)$ denotes the smallest possible number $f$ with the property that any $n$-point set $P \subset \mathbb{R}^{d}$ admits a weak $\epsilon$-net of cardinality $f$ with respect to convex sets.

Plugging the result of Theorem 1.1, and the previous planar bound [51, into (37) yields an improved bound in any dimension $d \geq 2$ :

$$
C_{d}(p, q)=O\left(p^{(d-1 / 2+\gamma)\left(1+\frac{d-1}{q-d}\right)}\right)
$$

for any constant parameter $\gamma>0$, and $p$ larger than a certain constant threshold which depends on $\gamma$.

- Our proof of Theorem 1.1 is fully constructive. The eventual net $N=N_{\text {vcon }} \cup N_{\text {surr }}$ includes the following main ingredients (over all the recursive sub-instances):

1. 1-dimensional strong $\lambda$-nets that are constructed via Theorem 3.7 for the lines $\ell \in$ $\mathcal{L}(r)$ with respect to the $\ell$-intercepts $\ell \cap \tau$ of the $(d-1)$-simplices $\tau$ in the restriction hypergraphs $\Pi \subseteq\left(\begin{array}{l}P \\ d\end{array}\right)$.

2. 1-dimensional strong $\lambda$-nets that are constructed in Section 4.2 (as part of $N_{t r}$ ) for the lines $\ell \in \mathcal{L}(s)$ with respect to the $\ell$-intercepts of the "mixed" $(d-1)$-simplices in $\left(\begin{array}{c}P \\ d-1\end{array}\right) * X$. Here each set $X$ is comprised of the $\ell^{\prime}$-intercepts of the sampled hyperplanes of $\mathcal{R}$, for $\ell^{\prime} \in \mathcal{L}(s)$, and of the vertices of the induced cutting $\mathcal{D}_{z}(\mathcal{R})$.

3. 1-dimensional strong $\lambda$-nets that are constructed in Sections 5.2 and 5.5 (as parts of the respective nets $N_{\text {sparse }}$ and $\left.N_{\text {loose }}\right)$ for the vertical lines $\ell \in \mathcal{L}_{i}(u)$ with respect to the $\ell$-intercepts of the $\tilde{O}\left(t^{d}\right)(d-1)$-simplices of $\bigcup_{i=1}^{s}\left(\begin{array}{c}V_{i} \\ d\end{array}\right)$, or of $\left(\begin{array}{c}\hat{V} \\ d\end{array}\right)$. Each set $V_{i}$ or $\hat{V}$ is comprised of the $\tilde{O}(t)$ vertices of the possibly clipped $d$-simplices $\varphi_{i, j}$ in the partition $\mathcal{Q}(s, t)$ of Section 5.2 .

4. Strong $\hat{\epsilon}$-nets that are constructed in Section 5.2 with respect to convex $(2 d)$-hedra (and the underlying point set $P$ ) using the Strong Epsilon Net Theorem 2.3

As the primary focus of this study is on the quantity $f_{d}(\epsilon)$, we did not seek to optimize the construction cost of our net $N$. A naive implementation of the recursive construction runs in time $O\left(n^{d+\alpha}\right)=\tilde{O}\left(n^{d}\right)$, for any $\alpha>0$, which is dominated by the non-recursive overhead spent on maintaining the restriction hypergraph $\Pi \subseteq\left(\begin{array}{l}P \\ d\end{array}\right)$ and tracing the zones of the hyperplanes of $\mathcal{H}(\Pi)$ within the cutting $\mathcal{D}_{z}(\mathcal{R})$ in Section 4.1. This also accounts for the 
cost of constructing the 1 -dimensional nets of Theorem 3.7 with respect to the $\ell$-intercepts $\ell \cap \tau$, for $\tau \in \Pi$ and $\ell \in \mathcal{L}(r)$.

Notice that all of the invocations of the vertical simplicial $r$-partition $\mathcal{P}(r)$ and the Multiple Selection Theorem 3.3 involve $r \lll 1 / \epsilon^{d-1 / 2} \ll n$. Hence, all the vertical decompositions $\mathcal{P}(r)$ and the vertical line sets $\mathcal{L}(r)$ in the applications of Theorem 3.3 can be obtained in $\tilde{O}(n)$ time via the Simplicial Partition Theorem 2.2. The same holds true for the construction of the cutting $\mathcal{D}_{z}(\mathcal{R})$ and the assignment of the points of $P$ to its cells in Section 4.1 [19]. As Matoušek's theorem [43] holds for any $1 \leq r \leq n$, the two-level partition $\mathcal{Q}(s, t)$ in Section 5.2 can be constructed in similar time $\tilde{O}(n)$. Armed with the partition $\mathcal{Q}(s, t)$, the set $\Pi_{\text {tight }}$ in Section 5.4 can be obtained in $O\left(|\Lambda| \times(s t)^{d-1}+n^{d}\right)=O\left(n^{d}\right)$ time.

\section{References}

[1] N. Alon, I. Bárány, Z. Füredi and D. J. Kleitman, Point selections and weak $\epsilon$-nets for convex hulls, Comb. Prob. Comput. 1 (1992), 189-200.

[2] N. Alon, A non-linear lower bound for planar epsilon-nets, Discrete Comput. Geom. 47 (2012), 235-244.

[3] N. Alon and G. Kalai, Bounding the Piercing Number, Discrete Comput. Geom. 13 (1995), $245-256$.

[4] N. Alon, G. Kalai, R. Meshulam, and J. Matoušek. Transversal numbers for hypergraphs arising in geometry, Adv. Appl. Math 29 (2001), 79--101.

[5] N. Alon, H. Kaplan, G. Nivasch, M. Sharir and S. Smorodinsky, Weak $\epsilon$-nets and interval chains, J. ACM 55 (6) (2008), Article 28.

[6] N. Alon and D. J. Kleitman, Piercing convex sets and the Hadwiger-Debrunner (p,q)-problem, Adv. Math. 96 (1) (1992), 103-112.

[7] B. Aronov, E. Ezra and M. Sharir, Small-size epsilon nets for axis-parallel rectangles and boxes, SIAM J. Comput. 39 (2010), 3248-3282.

[8] B. Aronov, J. Matoušek and M. Sharir, On the sum of squares of cell complexities in hyperplane arrange- ments, J. Combin. Theory Ser. A. 65 (1994), 311-321.

[9] B. Aronov and M. Sharir, Cell Complexities in Hyperplane Arrangements, Discrete Comput. Geom. 32 (2004), 107--115.

[10] B. Aronov, M. Pellegrini and M. Sharir, On the zone of a surface in a hyperplane arrangement, Discrete Comput. Geom. 9 (2) (1993), 177 - 186.

[11] J. Balogh, R. Morris and W. Samotij, Independent sets in hypergraphs, J. AMS, 28 (2015), $669-709$.

[12] J. Balogh and J. Solymosi, On the number of points in general position in the plane, Disc. Analysis 16 (2018), 1 - 20.

[13] I. Bárány, Z. Füredi and L. Lovász, On the number of halving planes, Discrete Comput. Geom. 10 (2) (1990), $175-183$. 
[14] P. V.M. Blagojević, B. Matschke, and Günter M. Ziegler, Optimal bounds for the colored Tverberg problem, J. Europ. Math. Soc. 17 (4), 2015, 739--754.

[15] H. Brönnimann and M. T. Goodrich, Almost optimal set covers in finite VC-dimension, Discrete Comput. Geom. 14(4) (1995), 463-479.

[16] B. Bukh, J. Matousek, and G. Nivasch, Lower bounds for weak epsilon-nets and stair-convexity, Israel J. Math. 182 (2011), 199-228.

[17] Ph. G. Bradford and V. Capoyleas, Weak epsilon-nets for points on a hypersphere, Discrete Comput. Geom. 18(1) (1997), 83 - 91.

[18] T. M. Chan, Optimal Partition Trees, Discrete Comput. Geom. 47 (2012), 661--690.

[19] B. Chazelle, Cutting hyperplane arrangements, Discrete Comput. Geom. 6 (1991), 385-406.

[20] B. Chazelle and E. Welzl, Quasi-optimal range searching in spaces of finite VC-dimension, Discrete Comput. Geom. 4 (1989), 467-489.

[21] B. Chazelle, The discrepancy method: randomness and complexity, Cambridge University Press, New York, NY, USA, 2000.

[22] B. Chazelle, H. Edelsbrunner, M. Grigni, L. J. Guibas, M. Sharir and E. Welzl, Improved bounds on weak epsilon-nets for convex sets, Discrete Comput. Geom. 13 (1995), 1-15. Also in Proc. 25th ACM Sympos. Theory Comput. (STOC), 1993.

[23] B. Chazelle and J. Friedman, A deterministic view of random sampling and its use in geometry, Combinatorica 10 (3) (1990), 229-249.

[24] K. L. Clarkson, H. Edelsbrunner, L. J. Guibas, M. Sharir and E. Welzl, Combinatorial complexity bounds for arrangement of curves and spheres, Discrete Comput. Geom. 5 (1990), 99-160.

[25] K. L. Clarkson and K. R. Varadarajan, Improved approximation algorithms for geometric set cover, Discrete Comput. Geom. 37(1) (2007), 430-58.

[26] H. Edelsbrunner, E. Mücke, Simulation of simplicity: a technique to cope with degenerate cases in geometric algorithms, ACM Trans. Graph. 9 (1) (1990), 66-104.

[27] P. Erdős and M. Simonovits, Supersaturated graphs and hypergraphs, Combinatorica 3 (2) (1983), 181-192.

[28] D. Eppstein, Improved bounds for intersecting triangles and halving planes, J. Combin. Theory Ser. A 62 (1993), 176-182.

[29] G. Even, D. Rawitz and S. Shahar, Hitting sets when the VC-dimension is small, Inf. Process. Lett. 95(2) (2005), 358-362.

[30] J. Fox, M. Gromov, V. Lafforgue, A. Naor, and J. Pach, Overlap properties of geometric expanders, Journal für die reine und angewandte Mathematik (Crelle's Journal) 671 (2012), 49-83.

[31] J. Fox, J. Pach, and A. Suk, A polynomial regularity lemma for semialgebraic hypergraphs and Its applications in geometry and property testing, SIAM J. Comput. 45(6) (2016), 2199-2223. 
[32] H. Furstenberg and Y. Katznelson, A density version of the Hales-Jewett Theorem, J. Analyse Math., 57 (1991), 64-119, 1991.

[33] H. Hadwiger and H. Debrunner, Über eine variante zum Hellyschen satz, Archiv der Mathematik, 8(4) (1957), $309-313$.

[34] D. Halperin and M. Sharir, New bounds for lower envelopes in three dimensions, with applications to visbility in terrains, Discrete Comput. Geom. 12 (1994), 313-326.

[35] D. Haussler and E. Welzl, $\varepsilon$-nets and simplex range queries, Discrete Comput. Geom. 2 (1987), $127-151$.

[36] A. Holmsen, Large cliques in hypergraphs with forbidden substructures, to appear in Discrete Comput. Geom. (2020).

[37] A. Holmsen, D.-G. Lee, Radon numbers and the fractional Helly theorem, arXiv: 1903.01068, priprint, 2019.

[38] C. Keller and S. Smorodinsky, A New Lower Bound on Hadwiger-Debrunner Numbers in the Plane, in Proc. 2020 ACM-SIAM Symposium on Discrete Algorithms (SODA 2020), pp. 11551169 .

[39] C. Keller, S. Smorodinsky and G. Tardos, Improved bounds for Hadwiger-Debrunner numbers, Israel J. Math. 225 (2) (2018), 925 - 945. (Also in Proc. SODA 2017, pp. 2254 - 2263.)

[40] J. Kolmós, J. Pach and G. J. Woeginger, Almost tight bounds for epsilon-Nets, Discrete Comput. Geom. 7 (1992), 163-173.

[41] J. Matoušek, Epsilon-Nets and Computational Geometry, in New Trends in Discrete Computational Geometry, J. Pach (Ed.), Algorithms and Combinatorics, Berlin, 1993, pp. 69-89.

[42] J. Matoušek, Lectures on Discrete Geometry. Springer-Verlag, New York, 2002.

[43] J. Matoušek, Efficient partition trees, Discrete Comput. Geom. 8 (3) (1992), 315-334.

[44] J. Matousek and U. Wagner, New constructions of weak epsilon-nets, Discrete Comput. Geom. 32 (2) (2004), 195-206.

[45] J. Matousek, R. Seidel and E. Welzl, How to net a lot with little: small epsilon-nets for disks and halfspaces, Proc. 6th ACM Symp. Comput. Geom., 1990, pp. 16-22.

[46] S. Moran and A. Yehudayoff, On weak epsilon-nets and the Radon number, Proc. 35th Symp. Comput. Geom. 2019, pp. 51:1-51:14.

[47] N. H. Mustafa and S. Ray, Weak $\varepsilon$-nets have a basis of size $O(1 / \varepsilon \log 1 / \varepsilon)$, Comput. Geom. 40 (2008), $84-91$.

[48] N. H. Mustafa and K. Varadarajan, Epsilon-approximations and epsilon-nets, Chapter 47 in Handbook of Discrete and Computational Geometry, J.E. Goodman, J. O'Rourke, and C. D. Tóth (ed.), 3rd edition, CRC Press, Boca Raton, FL, 2017.

[49] G. Nivasch and Micha Sharir, Eppstein's bound on intersecting triangles revisited, J. Comb. Theory, Ser. A 116(2) (2009), 494-497. 
[50] J. Pach and G. Tardos, Tight lower bounds for the size of epsilon-nets, J. AMS 26 (2013), 645 -658 .

[51] N. Rubin, An improved bound for weak Epsilon-nets in the plane, in Proc FOCS 2018, pp. $224-235$.

[52] B. Tagansky, A new technique for analyzing substructures in arrangements of piecewise linear surfaces, Discret. Comput. Geom. 16(4), 455 - 479 (1996).

[53] D. Saxton and A. Thomason, Hypergraph containers, Inventiones Mathematicae 201 (3) (2015), $925-992$.

[54] M. Sharir, Almost tight upper bounds for lower envelopes in higher dimensions, Discrete Comput. Geom. 12 (1994), 327-345.

[55] M. Sharir and P. K. Agarwal, Davenport-Schinzel Sequences and Their Geometric Applications, Cambridge University Press, New York, 1995.

[56] E. Szemerédi and W. T. Trotter, Extremal problems in discrete geometry, Combinatorica 3 (3-4) (1983), $381-392$.

[57] V. N. Vapnik and A. Y. Chervonenkis, On the uniform convergence of relative frequencies of events to their probabilities, Theory Prob. Appls. 16 (1971), 264-280.

[58] R. Wenger, Helly-type theorems and geometric transversals, in Handbook of Discrete and Computational Geometry, 2nd Edition (J.E. Goodman and J. O'Rourke, Eds.), Chapman \& Hall/CRC Press, 2004, pp. 73-96.

[59] R. T. Živaljević and S. T. Vrećica, The colored Tverberg's problem and complexes of injective functions, J. Comb. Theory Ser. A 61 (2) (1992), 309-318.

\section{A Proof of Theorem 3.2}

We establish the theorem with $\beta_{d}=b^{d+1}$, for $b=2 d+2$. A theorem of Erdős and Simonovits [27] yields constants $D_{d}>0$ and $D_{d}^{\prime}>0$ with the following property: Any $(d+1)$-uniform hypergraph $(V, E)$, with $|V|=n$ and $|E| \geq h\left(\begin{array}{c}n \\ d+1\end{array}\right)$, contains at least $D_{d}^{\prime} h^{b^{d+1}} n^{(d+1) b}$ copies of the complete $d$-partite graph $K_{b, b, \ldots, b}$ provided that $D_{d} n^{-1 / b^{d}} \leq h \leq 1$. We define $C_{d}:=D_{d}^{\prime} /\left(2 b^{2 d+2}\right)$.

Let us now fix $(V, E)$ a $(d+1)$-uniform hypergraph, and its $\left(C_{d} h^{b^{d+1}} n\right)$-conforming embedding $\chi: V \rightarrow \mathbb{R}^{d}$, that satisfy the hypothesis of Theorem 3.2 . Assume first that $h \geq D_{d} n^{-1 / b^{d}}$.

By the $\left(C_{d} h^{b^{d+1}} n\right)$-conformity, at most $C_{d} h^{b^{d+1}} n^{b^{d+1}} b^{2 d+2}=D_{d}^{\prime} h^{b^{d+1}} n^{b^{d+1}} / 2$ of these copies encompass a pair of vertices $v \neq v^{\prime}$ so that $\chi(v)=\chi\left(v^{\prime}\right)$. Therefore, there must remain at least $D_{d}^{\prime} h^{b^{d+1}} n^{b^{d+1}} / 2$ copies of $K_{b, \ldots, b}$ that encompass a subset $V^{\prime} \subset V$ of $b(d+1)$ vertices so that $\chi(v) \neq \chi\left(v^{\prime}\right)$ whenever $v \neq v^{\prime}$.

Fix one of these latter copies $\left(V^{\prime}, E^{\prime}\right)$ of $K_{b, \ldots, b}$, where $V^{\prime} \subset V$ consists of $b(d+1)$ vertices that do not overlap under $\chi$. Specifically, there is a partition $V^{\prime}=V_{1}^{\prime} \uplus V_{2}^{\prime} \uplus \ldots \uplus V_{d+1}^{\prime}$ so that $\left|V_{i}^{\prime}\right|=b$ for all $1 \leq i \leq d+1$, and $E^{\prime} \simeq V_{1}^{\prime} \times V_{2}^{\prime} \times \ldots \times V_{d+1}^{\prime} \subseteq E$. Let $P_{i}$ denote the set $\chi\left(V_{i}^{\prime}\right):=\left\{\chi(v) \mid v \in V_{i}^{\prime}\right\}$ for each $1 \leq i \leq d+1$. Then the improved variant [14] of the multi-colored Tverberg theorem [59] yields $d+1$ pairwise disjoint $(d+1)$-tuples $A_{1}, \ldots, A_{d+1} \in P_{1} \times P_{2} \times \ldots \times P_{d+1}$ so that $\bigcap_{i=1}^{d+1} \operatorname{conv}\left(A_{i}\right) \neq \emptyset$. 
Furthermore, by the general position of $\chi\left(V^{\prime}\right)$, the polytope $Q=\bigcap_{j=1}^{d+1} \operatorname{conv}\left(A_{j}\right)$ has non-empty interior.

We now argue that there exists $1 \leq i \leq d+1$, and selection of $d$ subsets $B_{j} \in\left(\begin{array}{c}A_{j} \\ d\end{array}\right)$ for $j \in[d+1] \backslash\{i\}$, so that the point $x:=\bigcap_{j \in[d+1] \backslash\{i\}} \operatorname{aff}\left(B_{j}\right)$ lies in the interior of $\operatorname{conv}\left(A_{i}\right)$. To this end, we consider any facet $F$ of the polytope $Q$. Note that $F$ is contained in a unique facet of a unique simplex conv $\left(A_{i_{1}}\right)$, for some $i_{1} \in[d+1]$. Assume with no loss of generality that $i_{1}=1$. In other words, we have that $F \subset \operatorname{conv}\left(B_{1}\right)$, for some $B_{1} \in\left(\begin{array}{c}A_{1} \\ d\end{array}\right)$. Then the $(d-1)$-dimensional polytope $Q_{1}=\operatorname{aff}\left(B_{1}\right) \cap \bigcap_{2<j<d+1} \operatorname{conv}\left(A_{j}\right)$ too has a non-empty relative $(d-1)$-dimensional interior. Thus, $Q_{1}$ has a $(d-2)$-dimensional facet $F_{1}$ of $Q_{1}$ which lies in a unique facet of a unique simplex conv $\left(A_{i_{2}}\right)$ for some $2 \leq i_{2} \leq d+1$. In other words, we have that $F_{1} \subseteq \operatorname{conv}\left(B_{i_{2}}\right)$, for some $B_{i_{2}} \in\left(\begin{array}{c}A_{i_{2}} \\ d\end{array}\right)$. Assume with no loss of generality that $i_{2}=2$, so the $(d-2)$-dimensional polytope $\operatorname{aff}\left(B_{1}\right) \cap \operatorname{aff}\left(B_{2}\right) \cap \bigcap_{i=3}^{d+1} \operatorname{conv}\left(A_{i}\right)$ has a non-empty $(d-2)$-dimensional interior and, thereby, a $(d-3)$-dimensional facet $F_{2}$. After $d$ steps, we end up with a 0 -dimensional polytope which can be assumed (with no loss of generality) to be of the form $x=\bigcap_{i=1}^{d} \operatorname{aff}\left(B_{i}\right) \cap \operatorname{conv}\left(A_{d+1}\right)$, for a suitable choice of $B_{i} \in\left(\begin{array}{c}A_{i} \\ d\end{array}\right)$. By the general position, we then have that $x=\bigcap_{i \in[d]}$ aff $\left(B_{i}\right)$, and $x$ lies in the interior of the simplex $\operatorname{conv}\left(A_{d+1}\right)$.

Let us now establish a lower bound for the number of $(d+1)$-tuples $\left(g_{1}, g_{2}, \ldots, g_{d}, f\right)$, with the following properties:

1. We have $f \in E$. Furthermore, there exist hyperedges $f_{i} \in E$, for $1 \leq i \leq d$, so that $g_{i} \in\left(\begin{array}{c}f_{i} \\ d\end{array}\right)$.

2. We have that $\left|\chi(f) \cup\left(\bigcup_{i=1}^{d} \chi\left(f_{1}\right)\right)\right|=d^{2}+d+1$. That is, the vertices of the $d+1$ hyperedges $f, f_{1}, \ldots, f_{d+1}$ do not overlap under the embedding $\chi: V \rightarrow \mathbb{R}^{d}$.

3. The point $\bigcap_{i=1}^{d}$ aff $\left(\chi\left(f_{i}\right)\right)$ lies in the interior of $\operatorname{conv}(\chi(f))$.

We have seen that every copy $\left(V^{\prime}, E^{\prime}\right)$ of $K_{b, \ldots, b}$ yields such a $(d+1)$-tuple $\left(g_{1}, g_{2}, \ldots, g_{d}, f\right)$, so that $g_{i}=\chi^{-1}\left(B_{i}\right)$ and $f=\chi^{-1}\left(A_{d+1}\right)$. Note that (i) the number of such copies $K_{b, \ldots, b}$ is at least $D_{d}^{\prime} h^{b^{d+1}} n^{b(d+1)} / 2$, whilst (ii) any $(d+1)$-tuple $\left(g_{1}, g_{2}, \ldots, g_{d}, f\right)$ that satisfies conditions (1) - (3), is shared by at most $B n^{b(d+1)-\left(d^{2}+d+1\right)}$ copies of $K_{b, \ldots, b}$, for a suitable constant $B>0$ that depends only on $d$. Hence, it readily follows that the number of the $(d+1)$-tuples that satisfy the above conditions $(1)-(3)$, is

$$
\Omega\left(\frac{C_{d} h^{b^{d+1}} n^{b(d+1)}}{B n^{b(d+1)-\left(d^{2}+d+1\right)}}\right)=\Omega\left(h^{b^{d+1}} n^{d^{2}+d+1}\right)
$$

Since the maximum number of the distinct $d$-tuples $\left(g_{1}, \ldots, g_{d}\right)$ is at most $n^{d^{2}}$, by the pigeonhole principle there must be a $d$-tuple $\left(g_{1}, \ldots, g_{d}\right)$ that participates in $\Omega\left(h^{b^{d+1}} n^{d+1}\right)$ of the above $(d+1)$ tuples $\left(g_{1}, \ldots, g_{d}, f\right)$, so the respective point $x_{0}:=\bigcap_{i=1}^{d}$ aff $\left(\chi\left(g_{i}\right)\right)$ pierces $\Omega\left(h^{b^{d+1}} n^{d+1}\right)$ of the simplices conv $(\chi(f))$. This confirms the theorem with $\beta_{d}=b^{d+1}$, and provided that $h \geq D_{d} n^{-1 / b^{d}}$.

Finally, if $h<D_{d} n^{-1 / b^{d}}$, then the theorem holds trivially, for we have that

$$
h^{b^{d+1}}\left(\begin{array}{l}
n \\
d
\end{array}\right)=O\left(n^{-b}\left(\begin{array}{l}
n \\
d
\end{array}\right)\right)=o(1)
$$

